\title{
Miniaturized Optical Components for Fluorescent DNA Detection System
}

\author{
A Thesis \\ Presented to \\ the faculty of the School of Engineering and Applied Science \\ University of Virginia
}

\author{
in partial fulfillment \\ of the requirements for the degree \\ Master of Science \\ by
}

Heather Marie Spence

May 2017 


\section{APPROVAL SHEET}

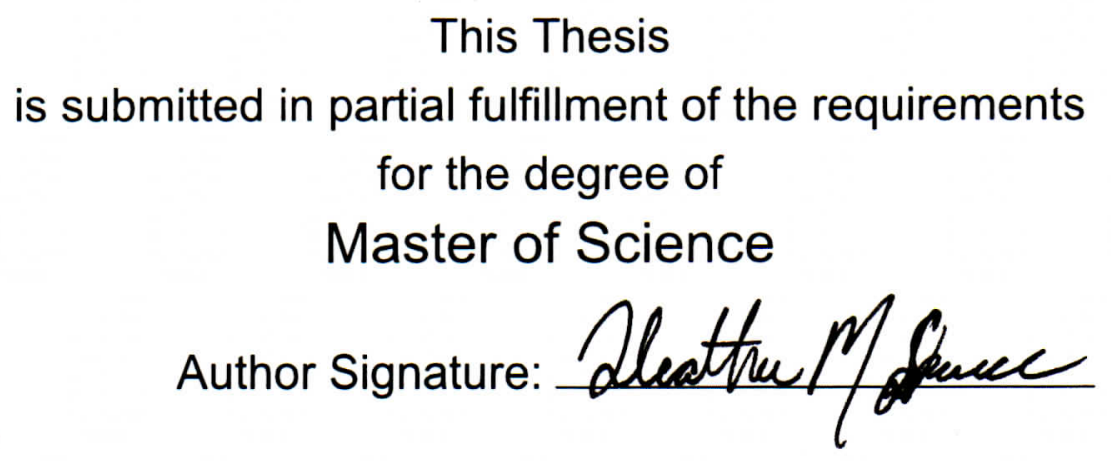

This Thesis has been read and approved by the examining committee:

Advisor: Andreas Beling

Committee Member: Joe Campbell

Committee Member: Stephen Wilson

Committee Member: James Landers

Committee Member:

Committee Member:

Accepted for the School of Engineering and Applied Science:

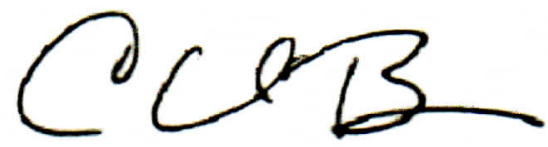

Craig H. Benson, School of Engineering and Applied Science

May 2017 
This thesis is dedicated

to my mother Melissa,

who has cheered me on through every accomplishment, reminding me to just breathe and bring it,

and

to my husband Travis,

for all the unwavering love and patience you have shown me;

thank you for always being my rock. 


\section{Abstract}

In a world that no longer sleeps, advances in technology are driven to provide accessibility and speed alongside accurate reliable data. Procedures previously designated to a lab, run by specially certified personal, now find niches among all facets of society by way of portable, rugged instrumentation coupled with intuitive graphical user interfaces. The demand for instrumentation to swiftly analyze human blood and tissue samples, especially among the medical field and military personnel, has driven research to focus on minimizing both instrument footprint and length of assay time.

Traditionally, fluorescence microscopy, while highly sensitive and able to provide precise data, is bulky and requires a full lab and great deal of time to analyze. The possibility of maintaining accurate data retrieval while also being able to identify fallen soldiers and enemies on the physical field of battle, or unidentified deceased in the hospital, near to real time has motivated a multitude of research in on-chip microfluidics using fluorescence detection schemes. The principle focus of research has centered on simplifying the polymerase chain reaction process, identifying fewer spots in the genome to approach ultra-rapid DNA screening times of thirty to forty-five minutes, maintaining multi-color color detection using instrumentation that is smaller and sturdier than photomultiplier tubes and solid state lasers, and using rotation-driven micro devices to integrate all microfluidics necessary onto one chip.

Given these goals, this thesis uses both computational simulations and experiments to explore alternatives to photomultiplier tubes as a primary detector and to characterize the laser diodes proposed over the traditional solid state lasers as excitation sources. Models of the 
optical components needed in detection as well as the in-house designed microscope objective are presented using Zemax OpticsStudio. In addition, three fully functional systems are presented and the changes made in designing each system are discussed. This body of work is considered the first step toward fully integrating and miniaturizing a rapid DNA screening device into the smallest footprint possible. 


\section{Acknowledgement}

First and foremost I would like to express my sincerest gratitude to my thesis advisor Prof. Andreas Beling. I could not have asked for a more supportive mentor, both personally and professionally; his advice and willingness to listen an invaluable gift these last three years. His unending faith in my abilities, even when I didn't believe in myself, gave me the strength and courage to pursue my dreams.

To Prof. Stephen Wilson and Beth Eastwood-Beatty, without whom none of this would be possible, thank you for every single letter written and every single question answered, making this scholastic journey just a little bit easier; you are my heroes.

To the rest of my advising committee: Profs. James Landers and Joe Campbell, thank you for your advice and support. This research has undoubtedly benefited from your teachings and guidance on the expectations of a superb research scientist.

I would also like to thank the Chemistry and Mechanical Engineering scientists within our collaborative group, who provided a plethora of mentoring and guidance throughout my studies Brian Root, Daniel Nelson, Brandon Thompson, Christopher Birch, Jingyi Li and Daniel Mills. In particular Daniel Nelson who guided me through the finer details of working in such a large team and was integral in the research and experimentation completed in chapter 3.

To my group members Dr. Min Ren, Ye Wang, Keye Sun, and Tiffany Ly, for assistance in my work and always being a friendly research sounding board. Especially Min Ren, for helping hunt down equipment and make my day a little brighter with a smile and fun family story. 


\section{Table of Contents}

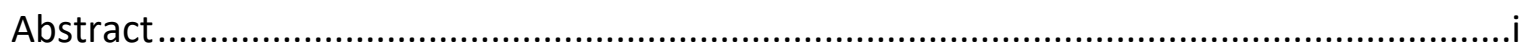

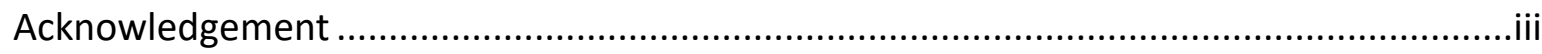

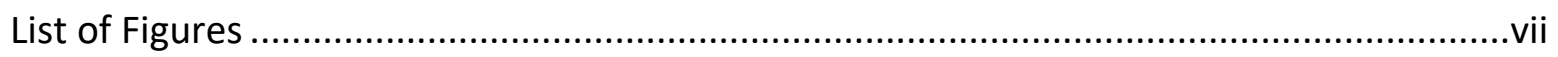

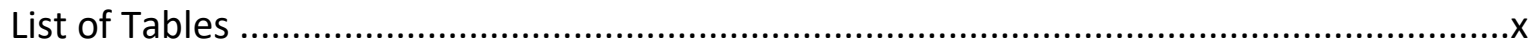

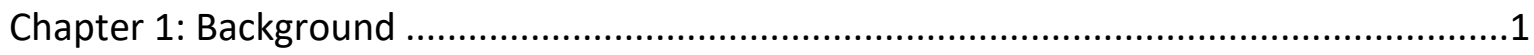

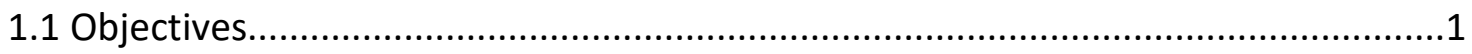

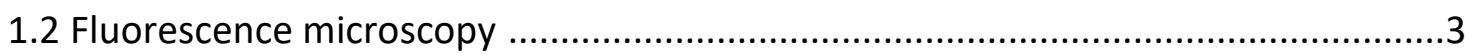

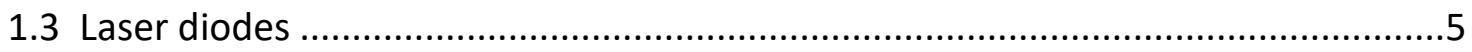

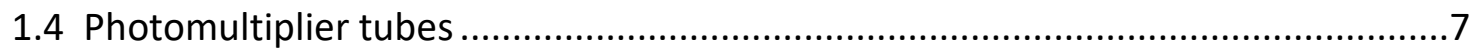

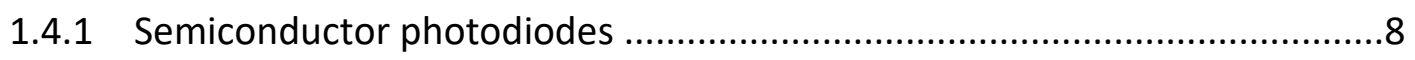

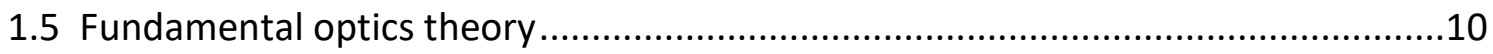

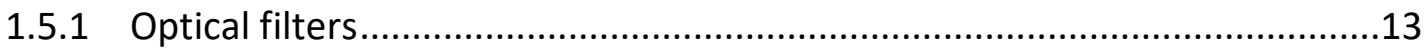

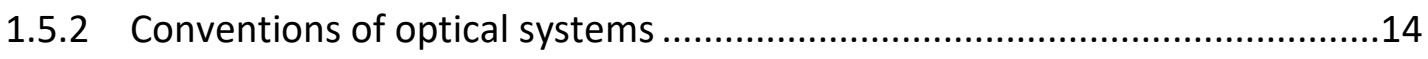

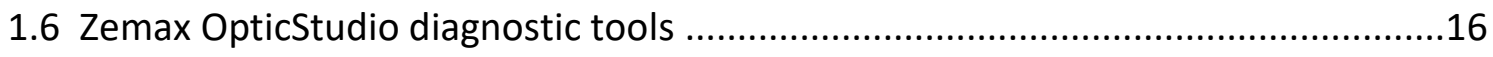

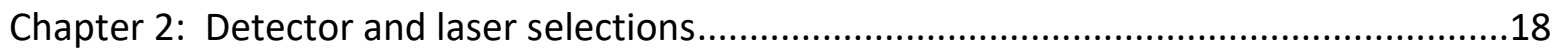

2.1 Summary of system laser specifications ......................................................

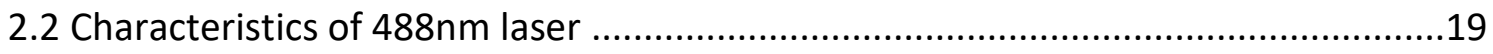

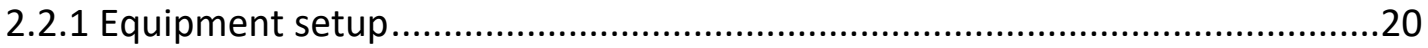

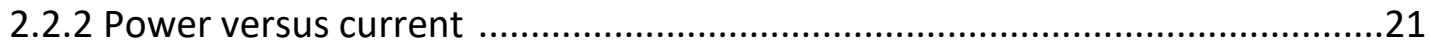

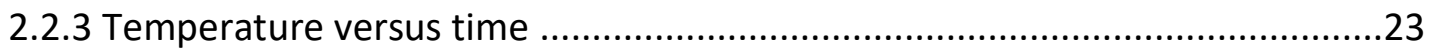




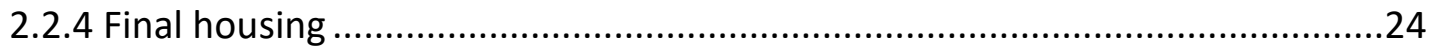

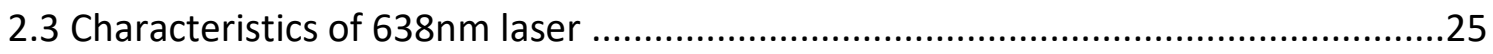

2.3.1 Temperature versus time for pulsed signal ................................................25

2.3.2 Temperature versus time for fluidic profile.....................................................27

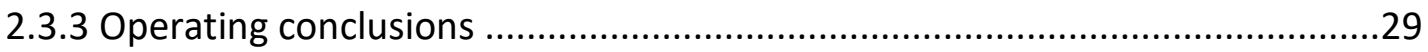

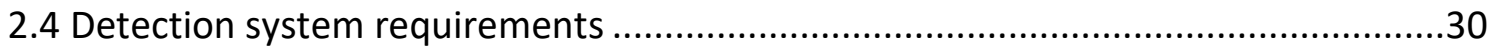

2.5 Exploring avalanche photodiodes as low-level light detectors .................................31

2.5.1 Current versus voltage and gain measurements .............................................32

2.5.2 External quantum efficiency measurements.................................................33

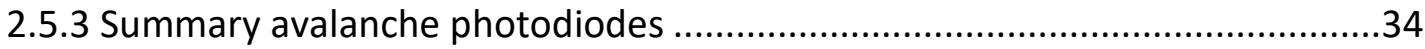

2.6 Exploring single channel MicroPMT module as detection system .............................35

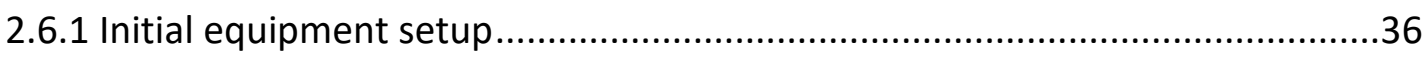

2.6.2 Testing microfluidic chip on benchtop system with MicroPMT .......................37

2.6.3 First integrated setup: Multi-Color System 1 ..................................................38

2.6.4 Pros and cons of Multi-Color System................................................................41

2.7 Linear array multianode PMT module as final detection system.............................41

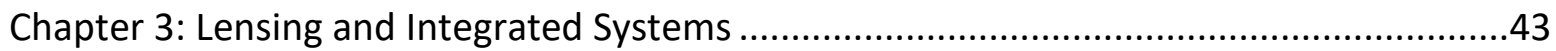

3.1 First optics modeling: $60 \mathrm{~mm}$ spherical lens with single color mPMT ......................43

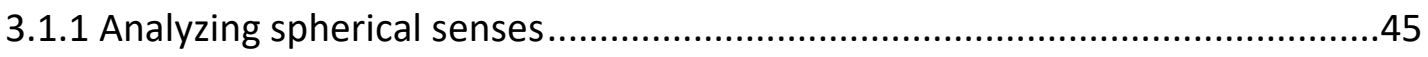

3.1.2 Microfluidic chip focus with spherical lens.....................................................46

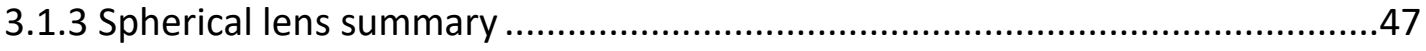

3.2 Second optics modeling: Multi-Color system $1 \ldots \ldots \ldots \ldots \ldots \ldots \ldots \ldots \ldots \ldots \ldots \ldots \ldots \ldots \ldots \ldots \ldots \ldots \ldots . . . .48$ 
3.2.1 Optical filter crosstalk

3.3 Third optics modeling: integrating and minimizing multi-color system $1 \ldots \ldots \ldots \ldots \ldots . . .51$

3.3.1 Laser placement and focus .53

3.4 Fundamental microscope objective structure and design criteria..........................54

3.4.1 Modeling for disassembled microscope objective ....................................56

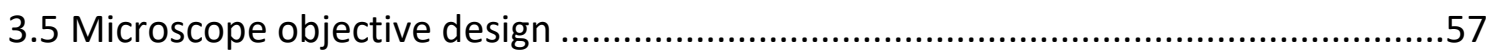

3.5.1 Microscope objective modeling iterations ..............................................62

Chapter 4: Conclusions and Future Work ..............................................................67

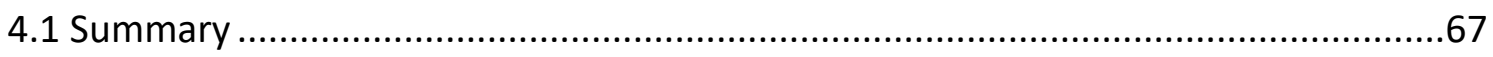

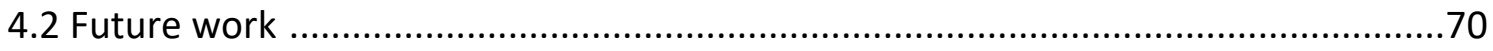

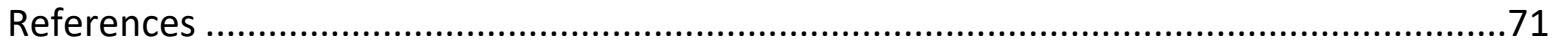




\section{List of Figures}

Figure 1-1. Excitation and emission spectra of fluorescein .........................................

Figure 1-2. Basic epifluorescence configuration for fluorescence microscopy..................4

Figure 1-3. Typical laser diode output power versus laser diode input current .................7

Figure 1-4. Physical structure and stages within photomultiplier tube ...........................

Figure 1-5. Plot of photodiode responsivity versus wavelength ..................................10

Figure 1-6. Optical ray diagram for converging and diverging lens ................................11

Figure 1-7. Cross sections of all the most common converging and diverging lens types....12

Figure 1-8. Optical ray diagram depicting focal point of spherical and aspherical lenses ....13

Figure 1-9. Surface of aspheric lens changing with radius of curvature ...........................13

Figure 1-10. Optical diagram with transmission and reflectance plots for

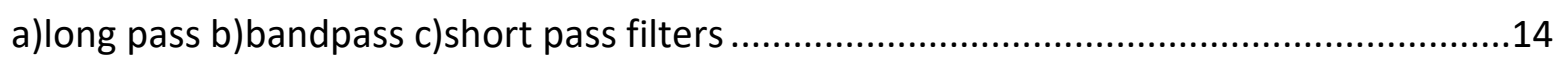

Figure 1-11. Cross section of single lens optic system and variables ...............................15

Figure 1-12. Ray fan plot model and characteristics ...............................................16

Figure 1-13. Ray and spot diagram for spherical aberration ......................................17

Figure 1-14. Ray fan plots for spherical aberration ...................................................17

Figure 2 -1. Laser diode mount and controllers ......................................................21

Figure 2-2. Experimental setup for laser diode power measurements...........................21

Figure 2-3. Plot of measured power versus current for $488 \mathrm{~nm}$ laser diode ......................22

Figure 2-4. Plot of 488nm laser power versus current from Osram data sheet .................22

Figure 2-5. Laser diode temperature versus time for a) $10 \mathrm{~m}$. trials b) $20 \mathrm{~m}$. trials ............23

Figure 2-6. Wavelength versus output optical power from $488 \mathrm{~nm}$ laser diode data sheet .23 
Figure 2-7. Experimental setup for testing $638 \mathrm{~nm}$ laser diode temperature .26

Figure 2-8. Temperature over time for $638 \mathrm{~nm}$ laser valve diode

Figure 2-9. Microfluidic profile timings for bursting each blister pack .28

Figure 2-10. Temperature measurements over fluidic profile without cooling 29

Figure 2-11. Temperature measurements over fluidic profile with cooling .29

Figure 2-12. Avalanche photodiode characteristics a) I-V plot b) gain versus voltage .33

Figure 2-13. Avalanche photodiode characteristics a) quantum efficiencyb) responsivity ..34

Figure 2-14. Initial equipment layout for testing mPMT .36

Figure 2-15. Current versus voltage characteristics of mPMT. .37

Figure 2-16. Filter, mirror and equipment layout for first multi-color system .38

Figure 2-17. Fluor color pathway breakdown in multi-color system one 39

Figure 2-18. Analyzed data retrieved from mPMT on multicolor system one .40

Figure 2-19. 3D Autocad designs for a) optical stack b) multicolor system two .42

Figure 3-1. Initial optical setup with spherical lenses a) top-down view b) actual

experimental setup .44

Figure 3-2. Model of single spherical lens with a collimated incident beam .45

Figure 3-3. Focusing laser into microfluidic chip a) no zoom b) zoomed and center

c) reflecting off bottom wall d) double lobe image..... .46

Figure 3-4. Model of spot size and shape for spherical lens .47

Figure 3-5. Optical transmission of FAM, JOE, ROX fluors versus wavelength .49

Figure 3-6. Fluor color pathway breakdown in multi-color system one .49

Figure 3-7. Plot of fluor signal on each mPMT. .50 
Figure 3-8. Optical component model for laser cylindrical lens stack .52

Figure 3-9. Spot diagram size and shape a) just after second cylindrical lens b) at chip ......53

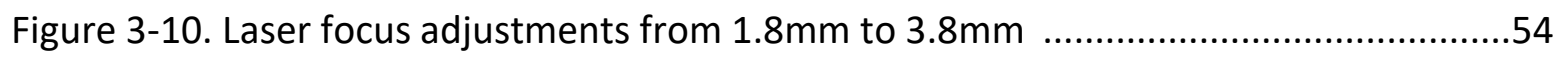

Figure 3-11. Numerical aperture versus working distance of microscope objective ...........55

Figure 3-12. Disassembled microscope objective data a) cross section ray diagram

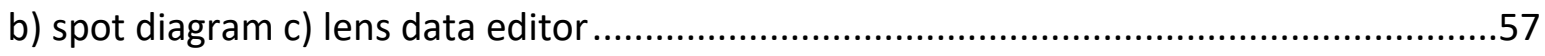

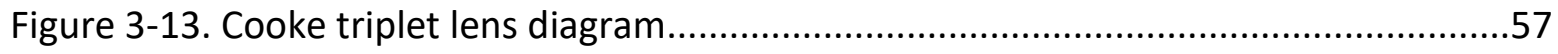

Figure 3-14. Designed Cooke triplet laser to chip a) ray diagram b) spot size ....................58

Figure 3-15. Designed Cooke triplet chip to detector a) ray diagram

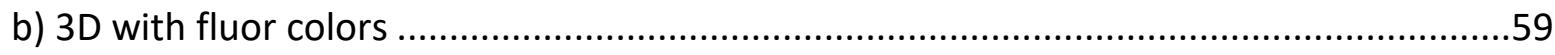

Figure $3-16$. Fully assembled in-house microscope objective ........................................60

Figure 3-17. Initial test setup for in-house microscope objective .................................60

Figure 3-18. Depiction of distances where beam diameter measurements were taken

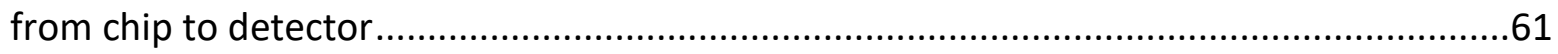

Figure 3-19. Distance from dichroic mirror versus emission beam diameter ....................61

Figure 3-20. First iteration of Condor optics a) ray diagram model b) spot size .................62

Figure 3-21. Second iteration of Condor optics a) ray diagram model b) spot size .............63

Figure 3-22. Third iteration of Condor optics a) ray diagram model b) spot size ...............64

Figure $3-23$. In-house objective focusing into chip ...................................................66

Figure 4-1. Year-end prototype assembly - Raptor .....................................................68

Figure 4-2. Top-down view of year-end prototype ................................................69

Figure 4-3. Power budget data for year-end prototype ..........................................69 


\section{List of Tables}

Table 2-1. Comparison of photodiode and avalanche photodiode characteristic

given from data sheet.

Table 2-2. Comparison of APD, PMT and mPMT critical characteristics given

from data sheet...

.35

Table 2-3. Comparison of data sheet specifications for MPMT and

linear array PMT.

Table 3-1. Comparison of potential aspheric lens for Condor optics .65 


\section{Chapter 1. Background}

Fluorescence spectroscopy is an interdisciplinary field utilizing software design, biological materials, chemical analysis and a variety of engineering concentrations including optical, electrical and mechanical subsets. The applications of fluorometric analysis include microorganism classification, such as various bacteria and fungi, drug identification, medical diagnosis and of course DNA screening for both forensic sciences and military research. The wide variety in applications can be attributed to the relative simplicity of this method while maintaining sensitivity, as well as the low cost in comparison to other analytical techniques. In most laboratory systems the detection of emitted light is recorded using photomultiplier tubes or charged-coupled devices given their ability to amplify incoming signals over a large detection range. That being said, their bulky, fragile nature makes them less than ideal for a portable device. A variety of techniques and instrumentation have been developed in order to account for this weakness, as well as address the weight and power consumption of solid state lasers that traditionally excite the sample after the polymerase chain reaction (PCR) process has taken place. An acute understanding of the underlying processes of fluorescent spectroscopy as well as the instrumentation needed for the system as a whole, will not only contribute to the recreation and improvement of existing techniques but also in the design of new systems, including both optical and mechanical design.

\subsection{Objectives}

The purposes of this thesis are to prove the viability of the proposed miniaturization mechanisms for creating an ultra-rapid DNA screening prototype that resembles the size and 
shape of a portable CD player, and deploys the mechanics of fluorescence spectroscopy. In doing so the characterization of each source and detection choice, as well as modeling of current and future optics necessary to achieve viable genome results are presented.

In the rest of this chapter, background knowledge regarding fluorescent microscopy and its use of fluorescent dyes to identify DNA, laser diodes, photomultiplier tubes and various other detectors, as well as important lensing and optics specifications are presented.

In Chapter 2, the specifications and needs of our detection system for the FaSTR project are outlined in regards to excitation source and photodetector. Following are characterizations of the Osram 488nm laser diode, chosen for its success as an excitation source in previous systems ${ }^{13}$, used in each evolved setup as well as power and temperature measurements for this, and the high power $638 \mathrm{~nm}$ laser used for breaking blister packs to release chemicals in the on-chip device. In addition, the minimum requirements needed of a new detection device, using data from Tan et al., are laid out. Given this information, three different electrical devices are investigated using a variety of simulations and experimental models to determine performance within required parameters. Resulting data acquired from each of the three evolved systems are described within their respective sections.

In Chapter 3, the corresponding optics and filters to each of the three systems are first modeled using Zemax OpticStudio and then demonstrated alongside excitation and detection devices using optical breadboards. Each setup includes data regarding focal length, spot size, and any major optical aberrations present within the system along with models used to determine how to best minimize aberrations and number of lens needed to reconcile emitted spectra on the detection face. Furthermore, each design theory and corresponding model used 
to create an in-house objective, along with focal length and spot size data are presented.

Finally, the year-end optical system using smallest optics, filters and electrical devices to date is modeled and presented alongside the final power budget and achieved detection limit.

\subsection{Fluorescence Microscopy}

In essence, fluorescence technology is based on the molecular absorption of light energy upon an illuminated specimen, and the nearly instantaneous emission of a separate, usually longer, wavelength than the excitation illumination. Many molecules will autofluoresce, without any application of fluorophores, given the correct excitation energy, while others can be modified, if needed, to fluoresce ${ }^{3}$. This more valuable process of adding fluorophore stains in order to attach to a very specific target have broadened the applications of fluorescent spectroscopy significantly, especially as new synthetic and more intense fluorophores are created $^{6}$. Modified or otherwise, each molecule contains two entirely unique characteristic spectra (see Fig. 1-1) referred to as the excitation spectrum (the wavelength of photons that are absorbed and needed to fluoresce) and emission spectrum (the longer wavelength emitted due to energy loss within the molecule). This emission of photons, through vibrational relaxation of the molecule, with less energy than the absorbed photon is also referred to as Stokes shift and is typically measured using a fluorescence microscope ${ }^{6}$.

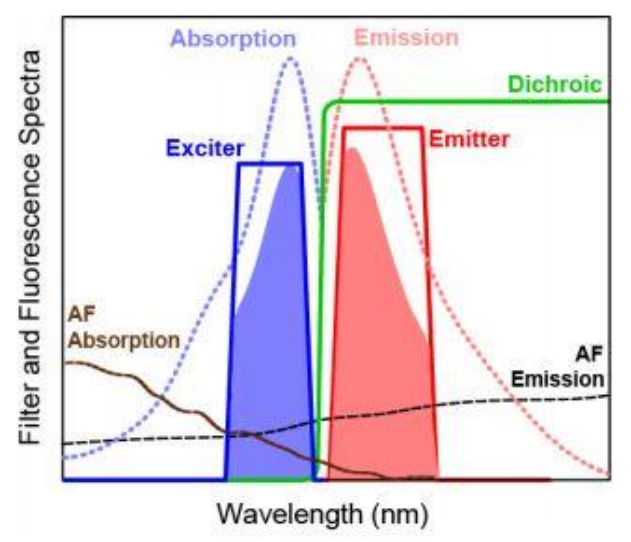

Figure 1-1. Excitation and emission spectra of fluorescein. ${ }^{1}$ 
Typically, fluorescence microscopes are arranged in the epifluorescence configuration (see Fig. 1-2), characterized by illuminating the sample in such a way that the microscope objective condenses light in both directions; focusing the excitation light onto the sample and then collecting the emitted fluorescence back through to the detector. Furthermore, this configuration is defined by placing the excitation light source $90^{\circ}$ to the emission path to reduce the risk of transmitted, or reflected, light reaching the detector, as well as utilizing multiple optical filters and dichroic mirrors to distinguish the two beams ${ }^{1}$. Furthermore, fluorescent dyes are easily multiplexed; allowing multiple specific dye colors to attach to different PCR primers, and while this allows for more markers within the genome results, it also requires combinations of more finessed filters to maintain a defined spectrum.

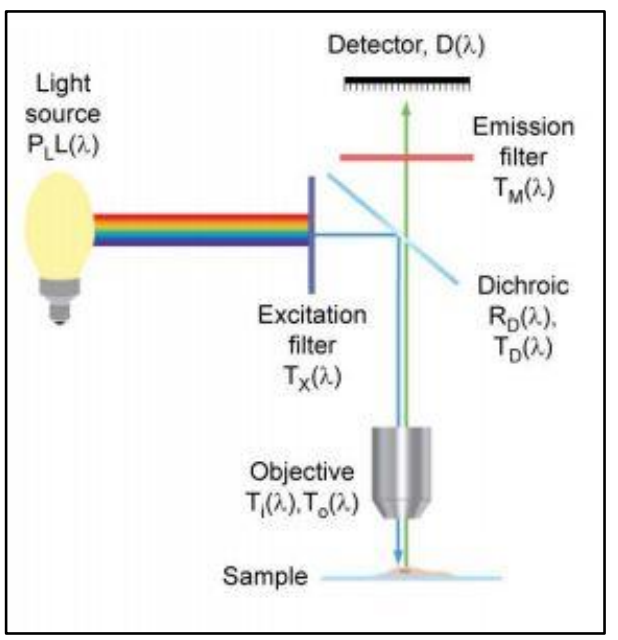

Figure 1-2. Basic epifluorescence configuration for fluorescence microscopy. ${ }^{1}$

Traditional excitation light sources are the Nd:YAG laser at 532 nanometers and Argon ion gas lasers, 488 to 514 nanometers, used in conjunction with photomultiplier tubes. The laser excites the fluorescence of the PCR product and then we electronically record the resulting wavelength and intensity, shape and color of the peaks. Many fluorescent dyes have 
overlapping spectra, meaning they will all reach maximum emission at slightly different excitation levels ${ }^{3}$. It is possible to excite them all with one lasing wavelength; this method simply requires more care in adjusting the concentration of each dyes in order to maintain equal levels of detection. Lasers provide an ideal light source in traditional fluorescence microscopy as they are very nearly monochromatic giving high power per spectral line and they have relatively long lifetimes. Regarding more mobile and cost efficient setups they provide a wealth of cons including high heat production, cost, and voltage requirements. One objective explored in this thesis is how to retain the positive characteristics of an ideal light source while also eliminating as many of the cons as possible.

\subsection{Laser Diodes}

Semiconductor lasers, also known as laser diodes, create powerful, precise beams of light, much like an ordinary solid state laser, but they are roughly the same size as simple light emitting diodes, used for instrument panels and lighting displays ${ }^{15}$. The semiconductor medium allows for great gains without complicated optics or fragile enclosures. Instead the laser cavity is composed from the two facets of the laser chip, what is known as a Fabry-Perot cavity. Crystal growth techniques are used for the epitaxial structure starting with an n-doped substrate and then growing the intrinsic active layer, ending with the $p$-doped layer and outermost contacts. This process creates a pn-junction where the incoming electrons combine with holes to create photons that bounce within the cavity, helping to create more photons through stimulated emission, while some of them are emitted through a partially reflecting facet. In many modern diodes, the main goal is to recombine all the carriers in the i-region so 
the innermost active layer also contains quantum wells in order to maximize chances for recombination and light generation, which allows the internal quantum efficiency of the laser to approach one output photon for each injected electron-hole pair.

The efficiency in converting electrical power to optical power is determined by the slope of the output light versus the injected current curve of the laser diode (see Fig. 1-3). The curve is measured in watts per amp and the linearity of the curve shows how close the specific diode comes to a one-to-one internal efficiency; ideally, the system will give out a great deal of power for very little input current. This curve may also show the separation between spontaneous emission, the emission of photons that occurs before the threshold, and stimulated emission, emission of photons after forward biased current is applied beyond threshold current.

The output beam of an edge-emitting laser diode, while concentrated in a small area, emits in an elliptical cone shape that is constantly diverging. The shape of the beam measured directly in front of the laser diode facet, the Near Field Pattern or NFP, is a horizontal oval shape in the $\mathrm{x}$-axis direction. The Far Field Pattern, or FFP, shape of the beam a few centimeters away from laser diode facet is still an ellipse but is horizontal in the $y$-axis direction ${ }^{14}$. The ellipse shape is attributed to the waveguide outlet in the semiconductor, which is fabricated narrower on the $y$ axis direction, causing the beam to diffract largely. The FFP is represented in data sheets by $\theta_{\|}$ and $\theta_{\perp}$. The $\theta_{\|}$indicates the angle at which the laser diode beam spreads horizontally, and the $\theta_{\perp}$ indicates the angle that the beam extends in the vertical direction ${ }^{14}$. The angle itself is defined by the full width at half maximum, where the intensity of the laser beam is half of the central portion of the laser beam. These values are typically $8^{\circ}$ for $\theta_{\|}$and $30^{\circ}$ for $\theta_{\perp}$. All laser 
diodes used in applications where collimated focus is desired throughout will require further lensing.

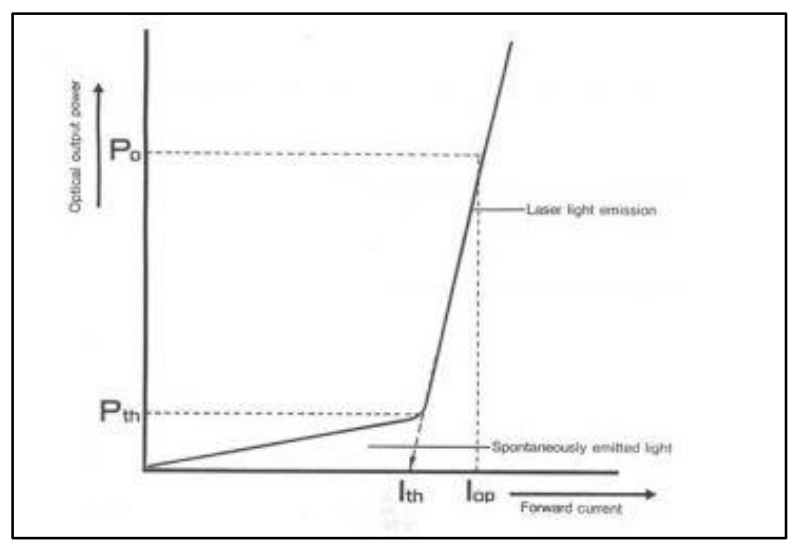

Figure 1-3. Typical laser diode output power versus laser diode input current. ${ }^{14}$

\subsection{Photomultiplier Tubes}

One of the more common types of photodetectors is the photomultiplier tube (see Fig. 1-4) characterized by a vacuum tube with anode, cathode, focusing electrodes and multiple dynode stages. It is a photoemissive device that uses focusing electrodes to catch as many electrons as possible; even ones that might hit along the outermost edges of the photocathode making it an ideal choice for sources that aren't fully coherent at the detector face ${ }^{8}$. Light incident on the photocathode results in the emission of electrons that are then amplified by electrodes, or metal channel dynodes, until finally collected at the end of the dynode chain by an anode, or collection electrode. The gain that can be achieved, with nearly noiseless amplification, varies with the voltage applied across dynode stages and the total number of dynodes present in the tube, generally giving a cumulative gain of $10^{4}$ through $10^{6}$, as well as very low dark current ${ }^{8}$. 


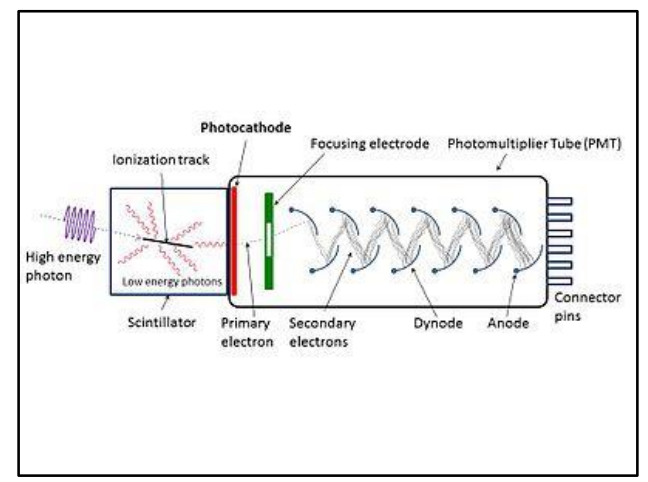

Figure 1-4. Physical structure and stages within photomultiplier tube. ${ }^{8}$

The photodetector is highly sensitive in the UV, visible, and near infrared ranges giving the possibility of detecting single photons; however, it is also incredibly sensitive to ambient light such that most systems must contain filters and external light shields. In addition, the gain mechanisms, number of dynode stages, makes the tube not only bulky but fragile and easy to break in field settings outside the laboratory. Furthermore, the photomultiplier tubes usually need very high operating voltages and in an integrated setting requires considerations be made to electric and magnetic shielding from other components as well as strict temperature control in order to maintain stable operations throughout ${ }^{8}$.

\subsubsection{Semiconductor Photodiodes}

Semiconductor photodiodes convert incident light into an electrical current, using a PN junction and the photoelectric effect for detection purposes. They are relatively cheap devices in comparison to a photomultiplier tube and are much smaller, similar to the size of laser diodes, with active areas up to $6 \times 6 \mathrm{~mm}$. Photodiodes are hardy and easily integrated with on chip devices, making them an obvious first choice as a replacement to the fragile, bulky photomultiplier tube. There are multiple types of photodiodes; the main focus of this thesis will be on P-N junction photodiodes and avalanche photodiodes, or APDs. When photons with 
energy greater than the semiconductor bandgap energy (in silicon: $1.1 \mathrm{eV}$ ) hit the diode, electron-hole pairs are created. If there is absorption in the depletion region, then the electronhole pairs are moved away from the junction, creating a current due to the holes moving towards the anode and electrons moving towards the cathode ${ }^{10}$.

The avalanche mode, characterized by electron-hole pairs knocking out other electron-hole pairs in a cascade effect, will give large amounts of internal gain which increases the sensitivity of the device, especially when operated in high reverse bias ${ }^{10}$.

The materials used in the production of the photodiode are critical to performance. The materials define the spectral range because only photons with sufficient energy to excite electrons across the bandgap will produce a photocurrent. The response ranges in silicon span from 190 nanometers to 1100 nanometers ${ }^{9}$.

The major characteristics to consider in choosing a photodiode are responsivity or quantum efficiency and the noise-equivalent power (NEP). The responsivity is the ratio of generated photocurrent to incident light power, given in amps per watts (see Fig. 1-5). Ideally, the detection system will have high responsivity at the desired wavelength and low responsivity elsewhere in the spectrum. The quantum efficiency is the ratio of the number of photogenerated carriers to incident photons. In avalanche photodiodes, a photoexcited carrier can produce extra electron-hole pairs as it accelerates through the depletion region. Due to these impact ionizations of extra electron-hole pairs, the total current in a photodiode is greater than what would be produced by photoionization alone ${ }^{10}$. This ratio of the total current to the primary current is represented as the internal current magnification, $M$, with a typical value ranging from $40-100$. The gain, $M$, is not constant, but rather an average value of a 
fluctuating process, and in order to account for this, the excess noise factor, $F$, is introduced which can typically range from 3 to $100^{9}$. In order to have a high signal to noise ratio, the photodetector must have a large quantum efficiency and gain in order to generate a large signal current. On the other hand the dark current and noise from the following electronic amplifier must also be as low as possible.

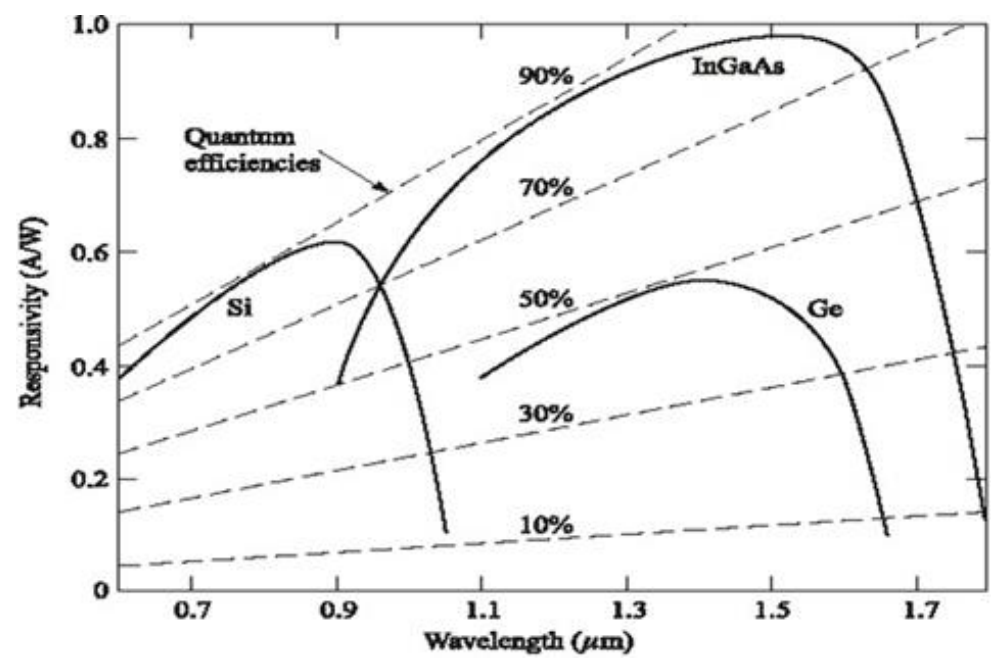

Figure 1-5. Plot of photodiode responsivity versus wavelength. ${ }^{10}$

\subsection{Fundamental Optics Theory}

In order to successfully build any detection system, a corresponding optical system must also be in place to collimate or collect and focus light signal as needed. Optical phenomena have been observed for more than 1000 years in nature before the Law of Refraction was derived in its current mathematical formula. The effects noticed in nature were clearly demonstrated through diagrams and experimentally reproduced using crystals to manipulate light and even magnify images. The modern theory of geometric optics begins with Galileo and his efforts in building a telescope in the $16^{\text {th }}$ century. The focus of research centered on creating 
lenses that held high surface quality in order to reduce the scattering effects and the use of homogeneous materials with little to no variations in the index of refraction ${ }^{4}$.

The basis of technical optics, the process by which an image is formed using a lens systems integrated with optical elements such as prisms and mirrors, begins with a clear understanding between the two basic types of lens: converging and diverging, shown in Fig. 1-6.

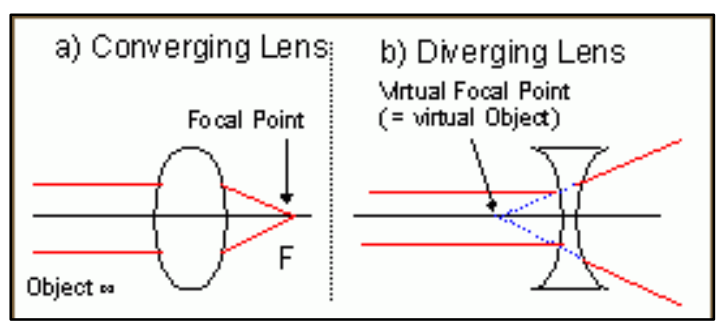

Figure 1-6. Optical ray diagram for converging and diverging lens. ${ }^{5}$

The converging lens has a positive refractive power and any collimated light traveling from infinity will pass through the lens and ideally come to a focused point, F, at some focal distance, $f$, from the center of the lens. A diverging point source will pass through a converging lens in the opposite direction and form a collimated beam of light. In contrast, the diverging lens has a negative refractive power and any collimated light traveling from infinity will pass through the lens and diverge upon exiting the opposite side. These rays do not focus to a point instead they form a projection of the virtual image point in the object space ${ }^{4}$. In both cases, a variety of lens cutting has been employed to give a wide variety of beam shaping in conventional optics systems, see Fig. 1-7. The most common lens types are the bioconvex, also known as a spherical lens, and bioconcave and meniscus lenses used in building microscope objectives and telescopes. All of these types are rotationally symmetric lenses, meaning that their refractive surfaces are spherical or conical and the outer diameter of the lens in concentrically ground. 


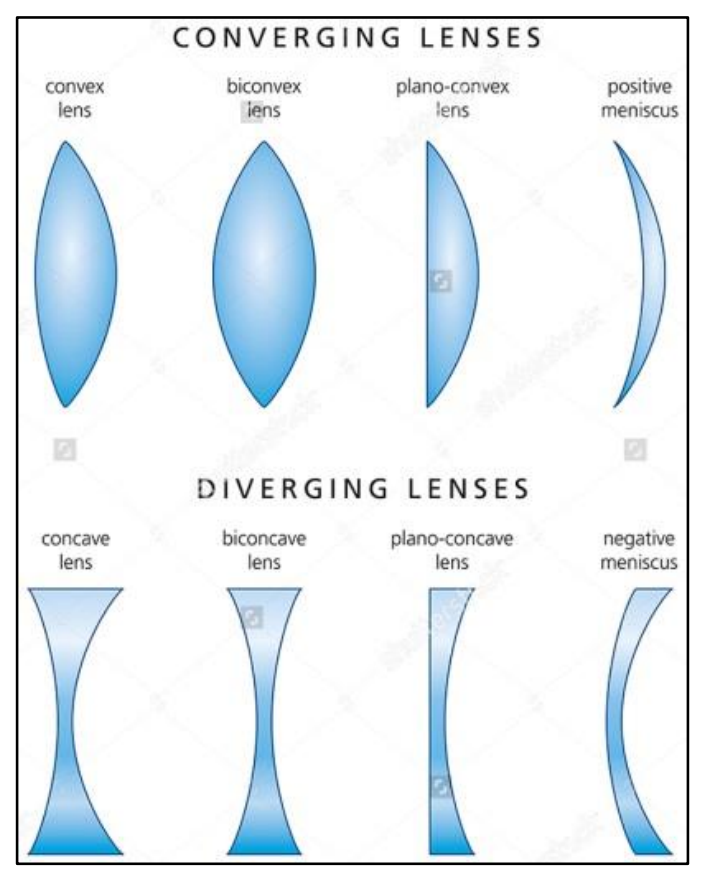

Figure 1-7. Cross sections of all the most common converging and diverging lens types. ${ }^{5}$

The next fundamental defining feature of a lens is the way in which the surface is shaped giving the optics either an aspheric surface or spherical surface. This designation refers directly to the lenses focusing ability and the amount of rays that are focused at the focal point of the lens ${ }^{5}$. A parallel coherent beam incident on a simple spherical lens, which holds a constant radius of curvature from the optical axis, will not focus all of the rays to the focal point, $F$. Instead the rays will be staggered out towards the image plane, centered on the focal point due to various levels of spherical aberration, see Fig. 1-8. The most unique geometric feature of the aspheric lens is that its radius of curvature changes with distance along the optical axis (see Fig. 1-9), allowing an incident parallel coherent beam to be fully reconciled to a focused point at the focal point, $\mathrm{F}^{5}$. 


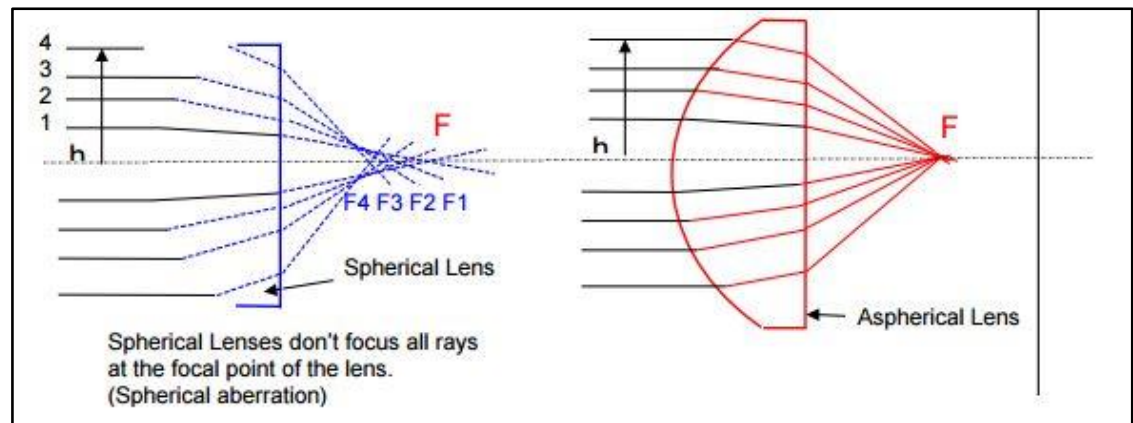

Figure 1-8. Optical ray diagram depicting focal point of spherical and aspherical lenses. ${ }^{5}$

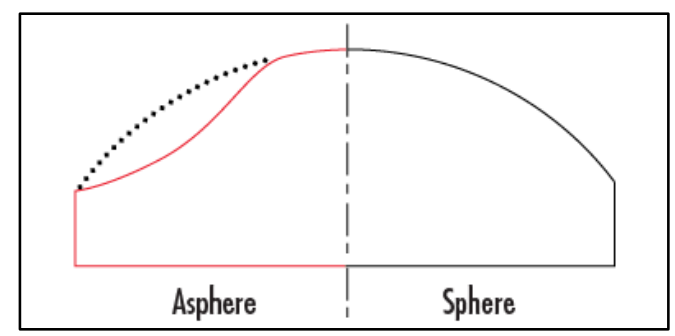

Figure 1-9. Surface of aspheric lens changing with radius of curvature. ${ }^{5}$

\subsubsection{Optical Filters}

In an optical system, filters are used to selectively transmit or reflect light of different wavelengths along different branches of the optical axis. They are a necessary piece of equipment in any detection system in order to have complete control of where each wavelength of light is being directed. They are usually made from plain glass or types of plastic and can be fabricated in a variety of shapes. The most common types are absorption filters, which absorb the undesired wavelength and transmit the all the others, and interference or dichroic filters. Dichroic filters are made by coating glass with a series of multilayered thin film coatings in order to reflect the unwanted light, while transmitting the rest ${ }^{1}$. They are also referred to as interference filters given that they employ the principle of interference in order to reflect unwanted wavelength. In short, the thin film layers for a sequential series of reflective 
cavities that resonate with the desired wavelengths and all the other wavelengths destructively cancel or reflect as the peaks and troughs dictate. The reflectance bands given in the data sheet would also show whether or not the filter is a longpass filter, one which attenuates short wavelengths and transmits longer ones as shown in Fig.1-10a, shortpass filter, one which attenuates longer wavelengths and transmits shorter wavelengths as shows in Fig. 1-10c, or finally a bandpass filter, shown in Fig. 1-10b, which only allows a range of wavelengths to pass.

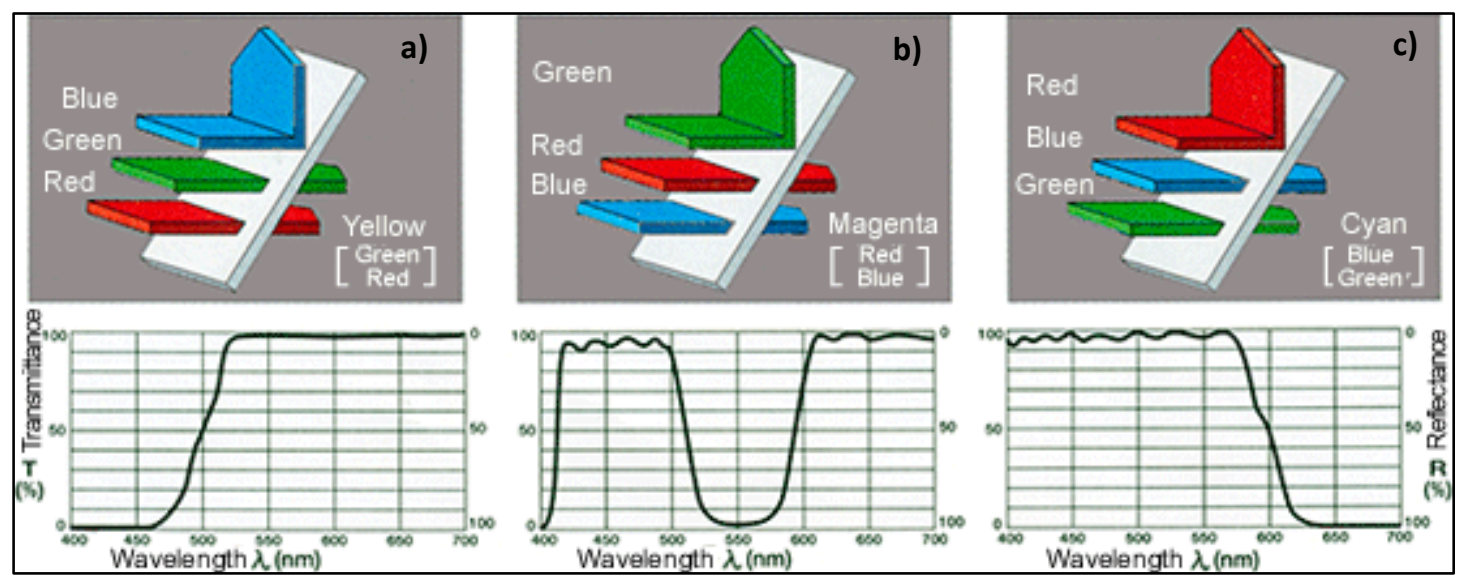

Figure 1-10. Optical diagram with transmission and reflectance plots for a)long pass b)bandpass c)short pass filters. ${ }^{3}$

\subsubsection{Conventions of Optical Systems}

All conventional optics systems implement the same important parameters to define the system. Traditionally the parameters are defined using a single lens system, or single step system, that illustrates the formation of a real image (see Fig. 1-11). A lens with focal length, $f$, and thickness, $t$, is placed on an axis, a known distance from the object of which an image will be created. The direction of propagation will always be defined from the object space moving 
towards the image space, giving the system an object distance, a, to the lens and an image distance, $a^{\prime}$, from the lens to the perceived image ${ }^{4}$.

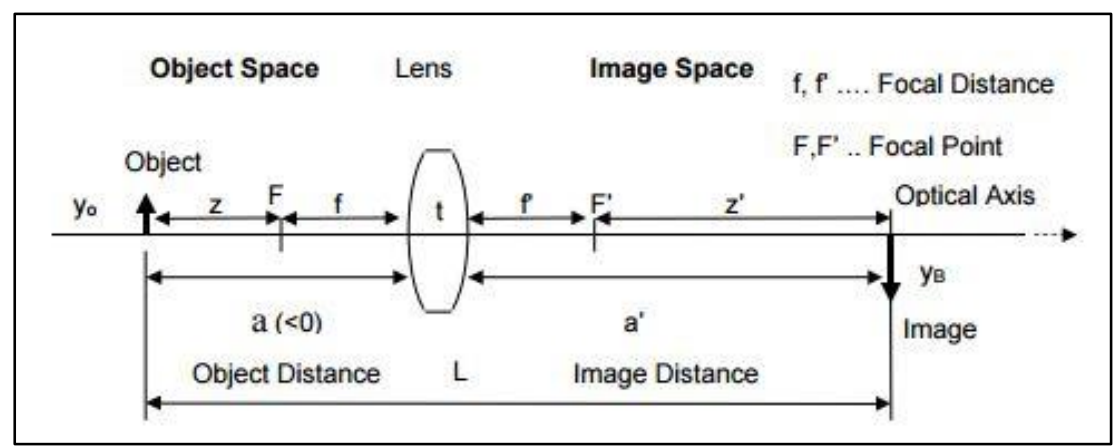

Figure 1-11. Cross section of single lens optic system and variables. ${ }^{4}$

The information that can be gleaned from a single step system provides all the parameters needed to further calculate the other important dimensional qualities needed to define a system: linear magnification, acceptance angle and the field of view, as well as extrapolate the information into a compound lens system ${ }^{4}$. The conjugate distance equation ${ }^{4}$ which defines the focal length of the lens in terms of the object and image distances is $\frac{1}{f}=\frac{1}{a^{\prime}}-\frac{1}{a}$. The linear magnification of the lens is also defined by the ratio of image and object distances as $m=\frac{a}{a \prime}$. The expressions for magnification and focal distance are calculated assuming they are calculated using only the paraxial rays whose angles are small relative to the optical axis, assuming $\theta=\sin \theta$. 


\subsection{Zemax OpticStudio Diagnostic Tools}

Zemax OpticStudio is a software platform that I used to simulate interactive visuals of each optical system. The most valuable diagnostic tools within Zemax are the Ray Fan Plot and Spot Diagram, both of which were used extensively to diagnose aberrations within the in-house optics designs. The Ray Fan Plot begins with a point source being launching rays toward the optic in the tangential plane, as shown in Fig. 1-12. The ray that pierces the center of the optic is designated as the chief ray; all others are some distance from that ray along the y-axis. The abscissa represents the $y$-axis of the pupil and the ordinate represents the $y$-axis of the image plane; both of which are normalized by the radius of the entrance pupil and then plotted together. The shape of the plot depends on the type and magnitude of the aberrations present in the system ${ }^{4}$.

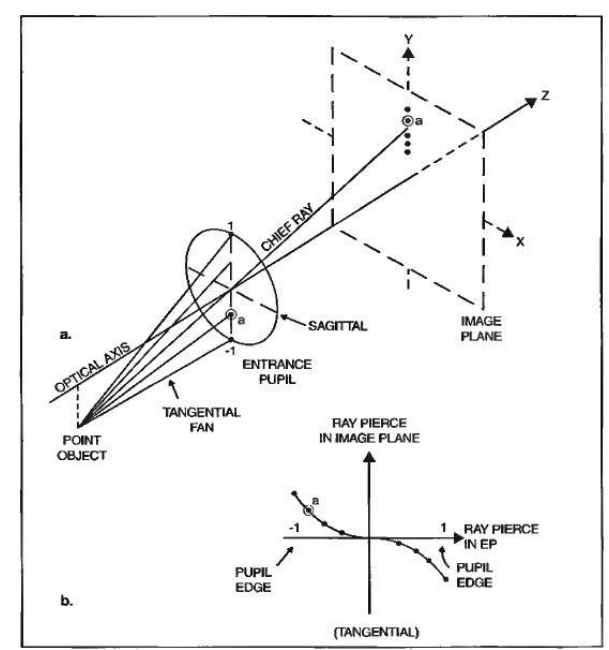

Figure 1-12. Ray fan plot model and characteristics. ${ }^{4}$

The Spot Diagram superimposes a uniform rectilinear grid, among other options, over the entrance pupil of the system before launching rays toward the image plane ${ }^{4}$. This gives a two dimensional distribution which will change depending on the specific aberration present in the 
system. In addition, the more compact the spot diagram is, the less aberration is present and furthermore, a perfect, ideal system with no aberrations would appear as a single point.

A few of the aberrations found within a system are defocus, where rays only cross at the paraxial focus and are slightly out of focus everywhere else along the optical axis and, coma, in which the optical components themselves are not aligned properly and most commonly found spherical aberration, a condition in which not all of the rays cross at the paraxial focus ${ }^{4}$. Instead some of the marginal rays will cross earlier creating a natural "waist" in the focus (see Fig. 113), creating what is referred to as the minimum blur point. The spot diagrams for spherical aberrations are no longer geometrically scaled; rather each one is unique as the image plane is moved in and out of the minimum blur point. The fan plots show a distinct " $\mathrm{S}$ " shape (see Fig. 114), which gives the magnitude of the spherical aberration present in the in the system. Each type of aberration can be diagnosed in a similar way using Zemax.

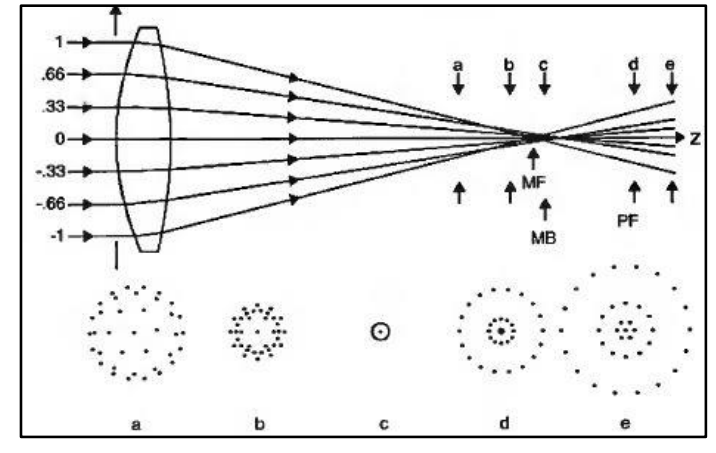

Figure 1-13. Ray and spot diagram for spherical aberration. ${ }^{4}$

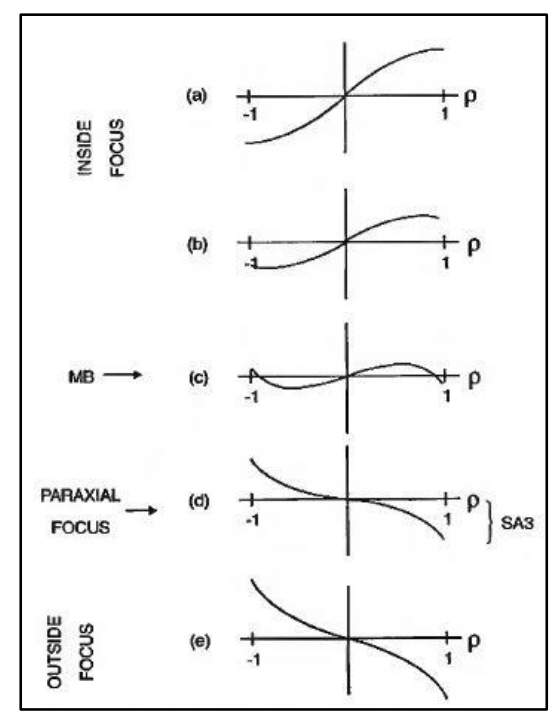

Figure 1-14. Ray fan plots for spherical aberration. ${ }^{4}$ 


\section{Chapter 2. Detector and Laser Selections}

In order to best decide which components to try and minimize first and what component would provide a viable choice moving forward, we reviewed previous work conducted by Shrinivasan et al, which proved a miniature laser-induced fluorescence detection system could successfully be built for single color using very minimal costs. This information allowed me to conclude that using laser diodes in place of traditional lasers would fit the system requirements quite well. In addition, it was decided that we would start with the smallest, cheapest option in place of the current photomultiplier tubes, a semiconductor photodiode. If successful, this would provide a hardy, easily integrated detector for the new system. In the course of our experimentation, it was decided that photodiodes ultimately wouldn't give the required gain to detect the minimum fluorescent signal over the achievable baseline. In place of the photodiodes, our team eventually chose to use the newer, compact, multiple channel micro-photomultiplier tubes within all of our evolved system setups. This section will provide information on the necessary specification of each laser and detector as well as the experimental data which prompted our team to either accept the new device into our systems or seek alternative equipment.

\subsection{Summary of System Laser Specifications}

The relative low cost of fabrication, high efficiency, high power and low threshold currents across a wide range of wavelengths make the laser diode an appropriate choice for replacing the standard laser within our fluorescence microscopy systems. In addition, with temperature control, we can monitor and adjust the temperature of the diode to ensure coherent, nearly 
monochromatic emission spectra and constant power for the duration of the experiment. With very minimal optics, the elliptical nature of the laser diode can be corrected over the short beam path so that the beam reaches the sample chip as collimated as possible. Simple optics also allows adjustment of the focus point size for the $488 \mathrm{~nm}$ light, so as not to oversaturate and photo bleach the fluorescent signal ${ }^{3}$, ruining the sample.

Furthermore, the FaSTR system required a high power pulsed laser diode in order to burn through and "burst" the blister packs of materials needed for different points within the PCR process. This diode required its own optics and temperature testing to ensure that it could be used in our system without active cooling, aside from fans and natural ventilation. Ideally the output power of the red laser would be high enough that the shortest possible dwell time, about one to three seconds, would be enough to burn the material and release the fluidics. Various tests were run at different operating currents to assess what input currents would provide the best trade off in output optical power and rising temperature concerns.

\subsection{Characteristics of $488 \mathrm{~nm}$ laser}

The blue single mode InGaN laser diode, purchased from OSRAM, was chosen to produce 488 nanometers because this wavelength would excite the various fluorescent dye colors as evenly as possible. The threshold current of the diode was $30 \mathrm{~mA}$ and the operating current was held constant at 90mA for each system. According to the data shown in Fig. 2-4, 90mA would produce the maximum amount of output optical power, $60 \mathrm{~mW}$. In addition to this, the data shown in Fig. 2-6, shows that in a constant temperature environment, we would expect very little change in the spectral width centering on 488 nanometers. The parallel beam divergence 
of this diode is $7^{\circ}$ while the perpendicular beam divergence is $23^{\circ}$ both values well within the general accepted region for laser diodes.

\subsubsection{Equipment Setup}

The equipment setup used to operate the blue laser diode remained the same through all of the early stages of the project. The setup consisted of a 488 continuous wave laser diode (output power $60 \mathrm{~mW}$ and case temperature $25^{\circ} \mathrm{C}$ ), laser diode mount with integrated thermoelectric coolers, a temperature control unit as well as a constant current control unit. The controllers and laser diode mount were purchased from Thorlabs as a precaution against ruining the laser diodes as the correct operating current and temperature were flushed out. The current control unit allowed a maximum current setting before it would shut off; this was normally set at $105 \mathrm{~mA}$ while the diode was traditionally held with a constant operating current of $90 \mathrm{~mA}$. Similarly the temperature control unit accepted a maximum temperature threshold that would cause it to shut down if crossed, this was set at $35^{\circ} \mathrm{C}$ and the laser diode mount itself held the diode and case at $25^{\circ} \mathrm{C}$.

The laser diode mount can handle multiple different pin configurations and holds its own safety measures so that ruining a diode, is very unlikely. It was also chosen because it was already fabricated to support focusing optics, screwed directly in front of the laser diode window. From the first experiment it was obvious that the lenses mounted on posts a few centimeters away from the diode would not be close enough to catch and reshape the wide elliptical nature of the beam. In order to start testing the detection sub systems, aspheric lenses 
were purchased from Thorlabs and fitted into the front middle of the diode mount; more on the motivating choices regarding the lens specifications will be explained in chapter three.

It's clear from Fig. 2-1 that this equipment was never meant to be a permanent solution as the mount and controllers are all very large, bulky and quite heavy. Rather the mount served as a protection while the specifications of the $488 \mathrm{~nm}$ laser were explored within our experiments; none of these pieces were implemented in the final design.

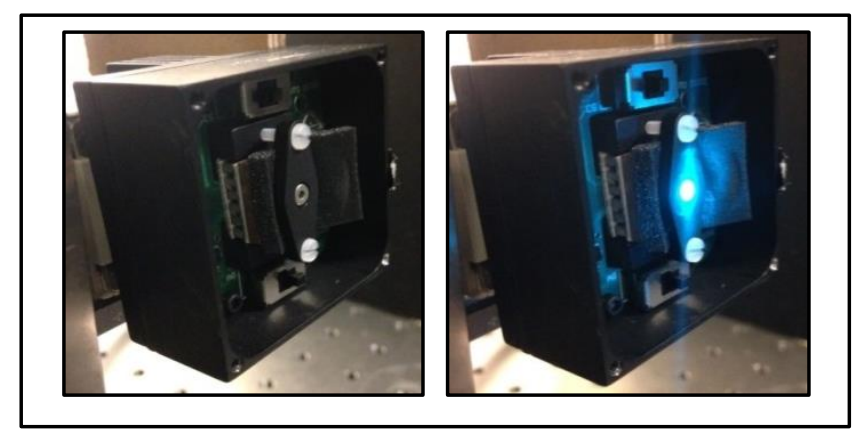

Figure 2-1. Laser diode mount and controllers.

\subsubsection{Power versus current}

First, before moving forward and using the laser diode with other equipment, it was necessary to confirm that each diode was in fact performing as expected based off of the information on the data sheet. In this setup, the $488 \mathrm{~nm}$ laser, nestled inside the laser diode mount was run using the constant temperature and current controllers. The diode was focused using identical two-inch diameter spherical plano-convex lenses, purchased from Newport,

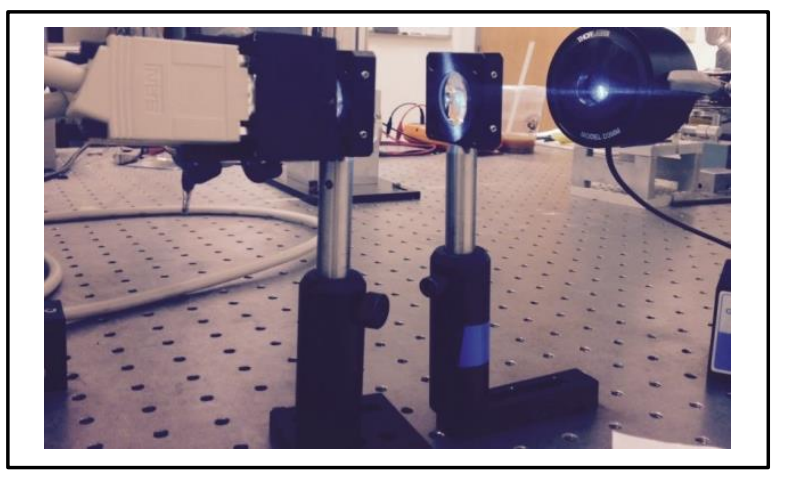

Figure 2-2. Experimental setup for laser diode power measurements 
which were placed in their own fixed mounts and lined up in the same beam path as the visible optical power meter as shown in Fig. 2-2. The focal point of both uncoated lenses was $60 \mathrm{~mm}$, so the distance they needed to be placed for as focused a point as possible on the power meter detection face, made them unsuccessful in catching and focusing the entire elliptical beam. The laser beam can be seen reflecting off of the second lens in Fig. 2-2 which proves the diode's beam is getting very large in that direction. At this point in time there was no other lensing in front of the laser, though as mentioned the diode mount has threading to purchase lenses and place them directly in front of the diode $(<1 \mathrm{~mm})$, which would give a much less divergent beam path. A more detailed answer regarding lens choice and design will be given in chapter three.

Once the equipment was secured to the optical table and the two lenses focused, the temperature controller is turned on and given time to stabilize. Once the temperature is stable, I slowly increased the input current, above the threshold current of $30 \mathrm{~mA}$, by increments of $5 \mathrm{~mA}$, giving it time to settle before increasing again. The experiment was run with the diode temperature held constant at $20^{\circ} \mathrm{C}$ and $40^{\circ} \mathrm{C}$ (see Fig. 2-3). The experimental data correlates nicely with the expected values from data sheet (see Fig. 2-4) and the diode is now ready to be used in determining the detection assembly.

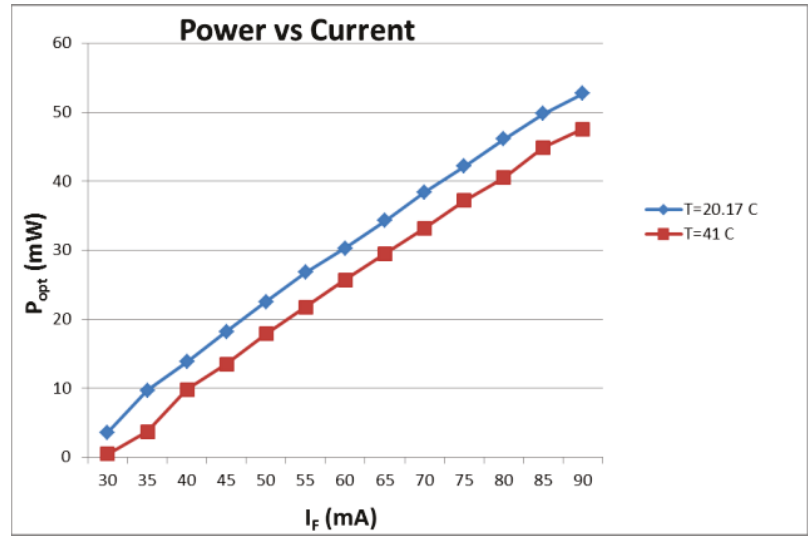

Figure 2-3. Plot of measured power versus current for $488 \mathrm{~nm}$ laser diode.

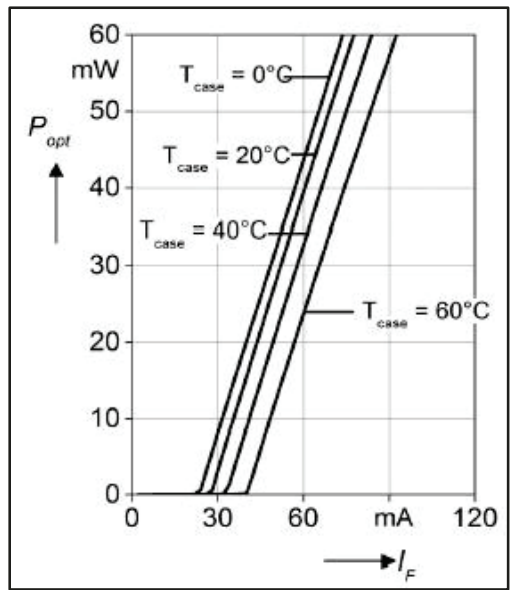

Figure 2-4. Plot of 488nm laser power versus current from Osram data sheet. ${ }^{16}$ 


\subsubsection{Temperature versus time}

In order to determine if we could remove the laser diode mount, controlling both the current and temperature, in order to reduce the amount of equipment and the sheer physical footprint of the system, the diode temperature profile needed to be made. The temperature testing would allow us to see exactly how the diode would perform when being taxed.

Each temperature experiment was conducted with the laser diode still in the laser diode mount; the thermoelectric coolers were just turned off in order to simulate a system with no active cooling. The operating current was set to $90 \mathrm{~mA}$ and each test, shown in Fig. 2-5a, was run for ten minutes with a five minute break in-between to allow the laser to cool to resting temperature. A final test (see Fig. 2-5b) was run for twenty minutes, without any pause, in an

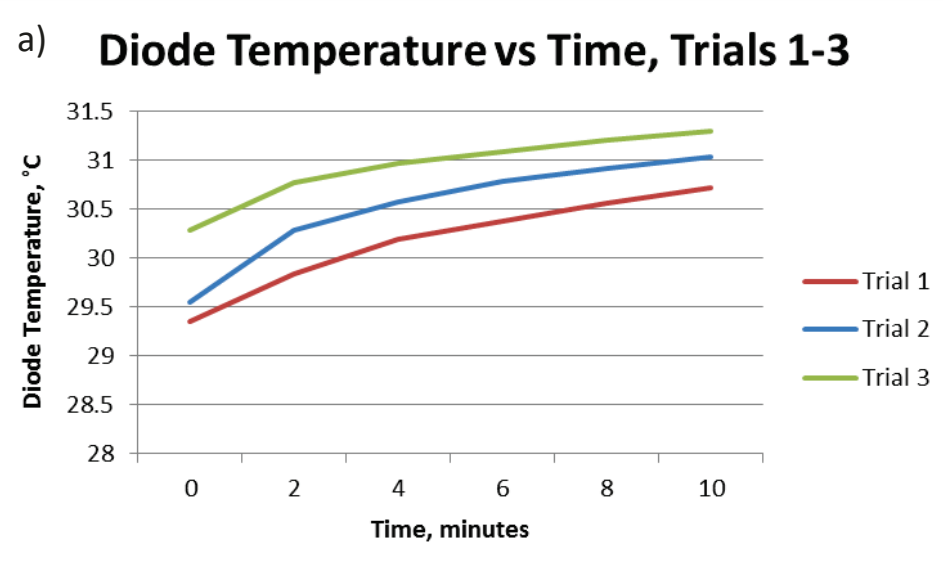

\section{b) Diode Temperature vs Time, Trial 4}
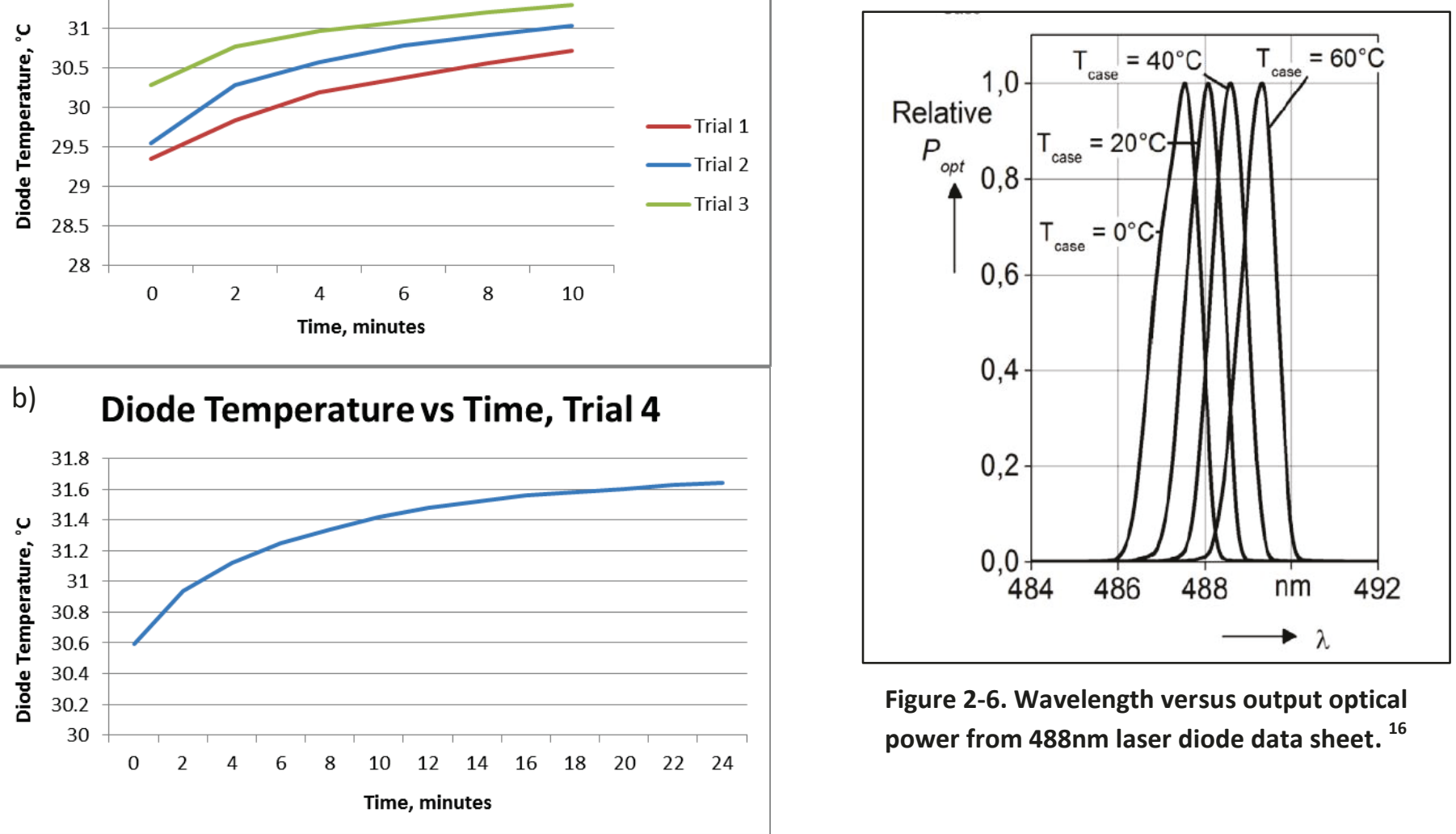

Figure 2-6. Wavelength versus output optical power from $488 \mathrm{~nm}$ laser diode data sheet. ${ }^{16}$

Figure 2-5. Laser diode temperature versus time for a) $10 \mathrm{~m}$. trials b) $20 \mathrm{~m}$. trials. 
attempt to identity any critical or break away point in the diode temperature. As shown in Fig.

2-5b, no breakaway temperature was ever observed; in fact at no point in the test did the diode temperature register above $32^{\circ} \mathrm{C}$, with change in temperature registering less than two degrees over twenty minutes of operation.

According to the spectra shown in Fig. 2-6, taken from the Osram data sheet, we can conclude that the change of emission wavelength with temperature is less than one nanometer. With this in mind, the laser diode was ready to be transferred from mount and controllers to the new housing, without constant temperature control, and optics.

\subsubsection{Final Housing}

All previous testing indicated that we could move the laser diode from the bulky, heavy and cumbersome mount and controllers into a new housing and optics setup. The temperature testing showed that as long as care was taken to give diode time to cool off between trials, it would never exhibit any breakaway temperature malfunctions which would in turn cause wavelength shifts potentially ruing the test. In addition to time between trials, a fan was installed directly perpendicular to laser diode in order to keep air moving across it.

As discussed, the laser diode mount and bulky controls helped not only control temperature and current, but gave a method to placing a collimating lens millimeters in front of the diode, maximizing focused signal to sample. The ability to shape the elliptical beam upon exiting the diode window was of paramount importance to ensuring a focused beam path; therefore, we researched housing methods which would also place a lens directly in front of the laser diode. In the end, the laser diode was placed inside an adjustable focusing and collimation tube, 
purchased from Thorlabs that fit directly into the optical rail system already in place. The tube ended in an aspheric lens with an antireflective coating for 350 - 700 nanometers.

In order to power the laser diode, strain relief cables with appropriate pin configurations were purchased from Thorlabs and wired directly into the power supply, an external bench power supply and eventually the PCB electronics bread board designed in house.

\subsection{Red Laser Characteristics}

The 638 nanometer red multi-mode AIGalnP laser valve diode, purchased from Thorlabs, was chosen for its high pulse frequency and capability to emit large amounts of energy per pulse. The threshold current of the diode was $250 \mathrm{~mA}$ and the operating current fell between $820-1000 \mathrm{~mA}$ depending on its intended use. According to the data operating as pulsed laser with input current between 820 - 10000mA would produce high output power pulses ranging from $550-700 \mathrm{~mW}$, depending on how the laser is used within the operating temperature, -10 to $+40^{\circ} \mathrm{C}$. The parallel beam divergence of this diode is $9^{\circ}$ while the perpendicular beam divergence is $35^{\circ}$, making them both a bit higher than the $488 \mathrm{~nm}$ laser but still well within the accepted region for the optics system.

\subsubsection{Temperature versus time for pulsed signal}

Once the 638 nanometer laser had been chosen, fellow group members Jingyi Li and Christopher Birch asked for assistance in characterizing the performance of the diode. In particular this diode would be placed either above or below the sample disk, and pulsed on for the duration of time it takes to burn through the "blister packs" of fluids that need to be 
released at various times in the PCR process. Originally the diode was paired with an aspheric lens that made it too focused and much too sensitive, even a few millimeters off would be enough to miss the blister pack completely. With the non-ideal optics, the laser focal point, regardless of the operating current and output power, was not allowing enough energy though to create a dense enough spot that would burn through the black polyester film in less than thirty seconds. Given that there would theoretically be twelve laser valves to burn through, with all of them taking at least thirty seconds, this stood to add at minimum six minutes to an already lengthy assay time. Ultimately, I adjusted the current aspheric lens into a wider focal point which allowed for a denser signal at the focal point, and began testing the temperature characteristics as they had asked.

I set up the red laser diode for temperature testing as shown in Fig. 2-7, taping the diode along the optic rails to hold it firm while measurements were taken. I used the same thermistor to monitor temperature inside the diode casing as with the 488 nanometer laser and began my testing with the diode outside of the housing without any active cooling. I pulsed the laser diode on for two seconds, the ideal amount of time it would take to burn through a blister pack, and then recorded temperature for sixty seconds, at every five second interval, over three different operating currents as seen in Fig. 2-8. I observed major changes in the laser temperature across the various operating currents, and even felt the temperature would have run away at input current, $750 \mathrm{~mA}$, if not turned off.
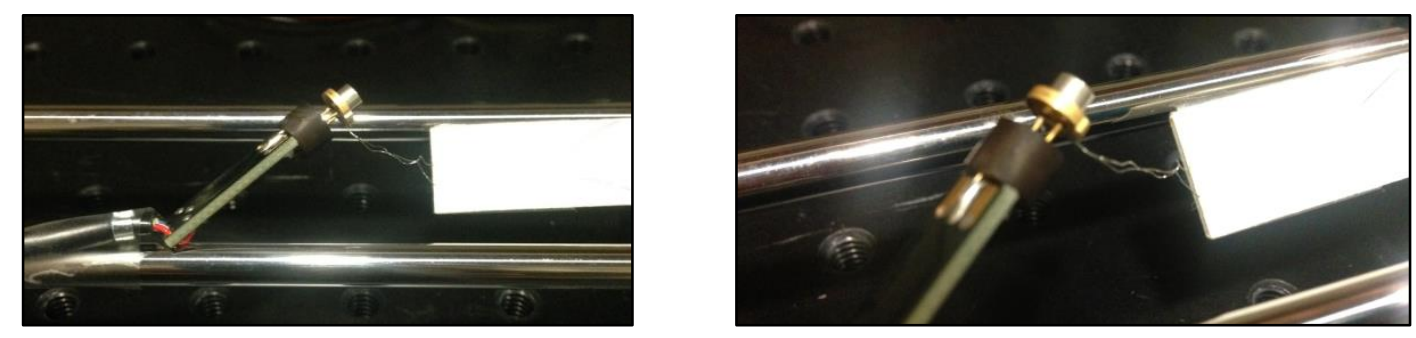

Figure 2-7. Experimental setup for testing $638 \mathrm{~nm}$ laser diode temperature. 


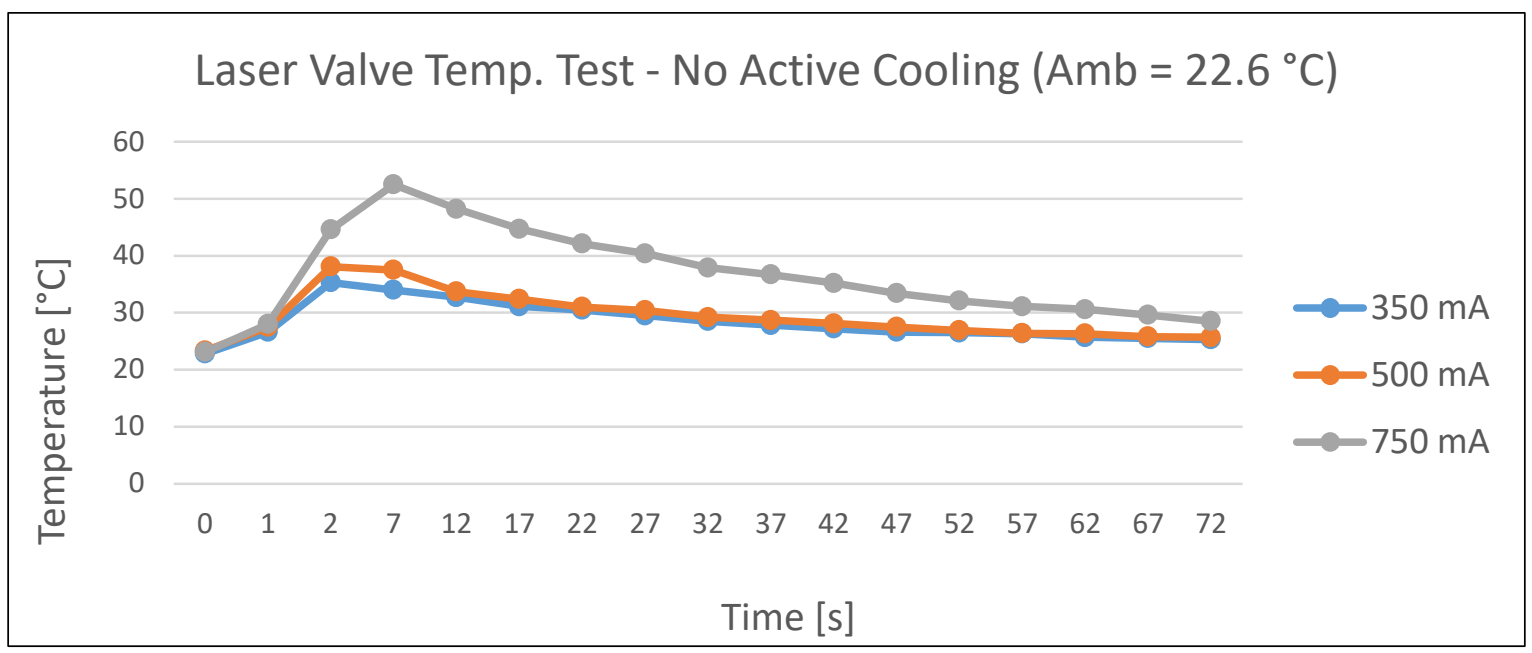

Figure 2-8. Temperature over time for $638 \mathrm{~nm}$ laser valve diode.

I repeated the same exact procedure after placing the laser diode back into the focusing tube with the thermistor probe bonded against the diode casing inside tube. The corresponding temperature readings showed very little fluctuation in the temperature over the course of the two-second pulse and sixty-second cooldown.

\subsubsection{Temperatures versus time for fluidic profile}

After completing initial temperature testing, I obtained a copy of the fluidic profile timings (see Fig. 2-9) from Christopher Burch and proceeded to run the red laser, inside and outside the focus housing, following these exact timings. All data was taken at an operating current of 500 $\mathrm{mA}$ with two seconds used to pulse for each valve. All rest times varied in accordance with their assignment from the proposed fluidic profile chart in Fig. 2-9.

As can be seen in Fig. 2-10, the experimental tests in which the laser was outside of the case without any active cooling, the diode spent majority of test above the safe operating range, 
$40^{\circ} \mathrm{C}$ (also shown with red bar). Operating consistently in this way would ruin and burn out the diode very quickly.

In the case where I tested the fluidic profile with the diode inside the focusing tube, in Fig. $2-11$, it's clear that laser spent most of test below the safe operating range, again indicated by red bar. It only approached the edge of the safe limit at the end of laser valves 4-6 which occur back to back without any rest time for the diode.

\begin{tabular}{|c|c|c|c|c|}
\hline Step & Speed(RPM) & Time (s) & Angle & Laser \\
\hline Move fluid from zygem chamber to swab chamber & 1000 & 30 & N/A & $\mathrm{N} / \mathrm{A}$ \\
\hline Extraction protocol & & & 275 & \\
\hline laser valve 1 & & & 212 & 31 \\
\hline Move fluid to aliquot chamber & 1500 & 60 & & \\
\hline Laser valve 2 & & & 202 & 37 \\
\hline Move aliquot to mixing chamber & 1500 & 20 & & \\
\hline Laser valve 3 & & & 250 & 32 \\
\hline Move PCR reagent to mixing chamber & 1500 & 5 & & \\
\hline Laser valve 4 & & & 228 & 40 \\
\hline Laser valve 5 & & & 280 & 36 \\
\hline Laser valve 6 & & & 281 & 41 \\
\hline Move mixed reagents to PCR chamber & 1500 & 20 & & \\
\hline Degas step protocol & & & 342 & \\
\hline PCR step & & & 342 & \\
\hline Laser valve 7 & & & 278 & 43 \\
\hline Transport PCR mix through & 2500 & 5 & & \\
\hline Laser valve 8 & & & 304 & 47 \\
\hline Transport to snap cool chamber & 2500 & 5 & & \\
\hline Laser valve 9 & & & 336 & 33 \\
\hline Transport snap cool & 1500 & 20 & & \\
\hline Laser valve 10 & & & 361 & 49 \\
\hline Transport sample to serparation domain & 2500 & 5 & & \\
\hline Polymer load & 2500 & & & \\
\hline Laser valve 11 & & & 433 & 42 \\
\hline Laser valve 12 & & & 449 & 42 \\
\hline
\end{tabular}

Figure 2-9. Microfluidic profile timings for bursting each blister pack. 


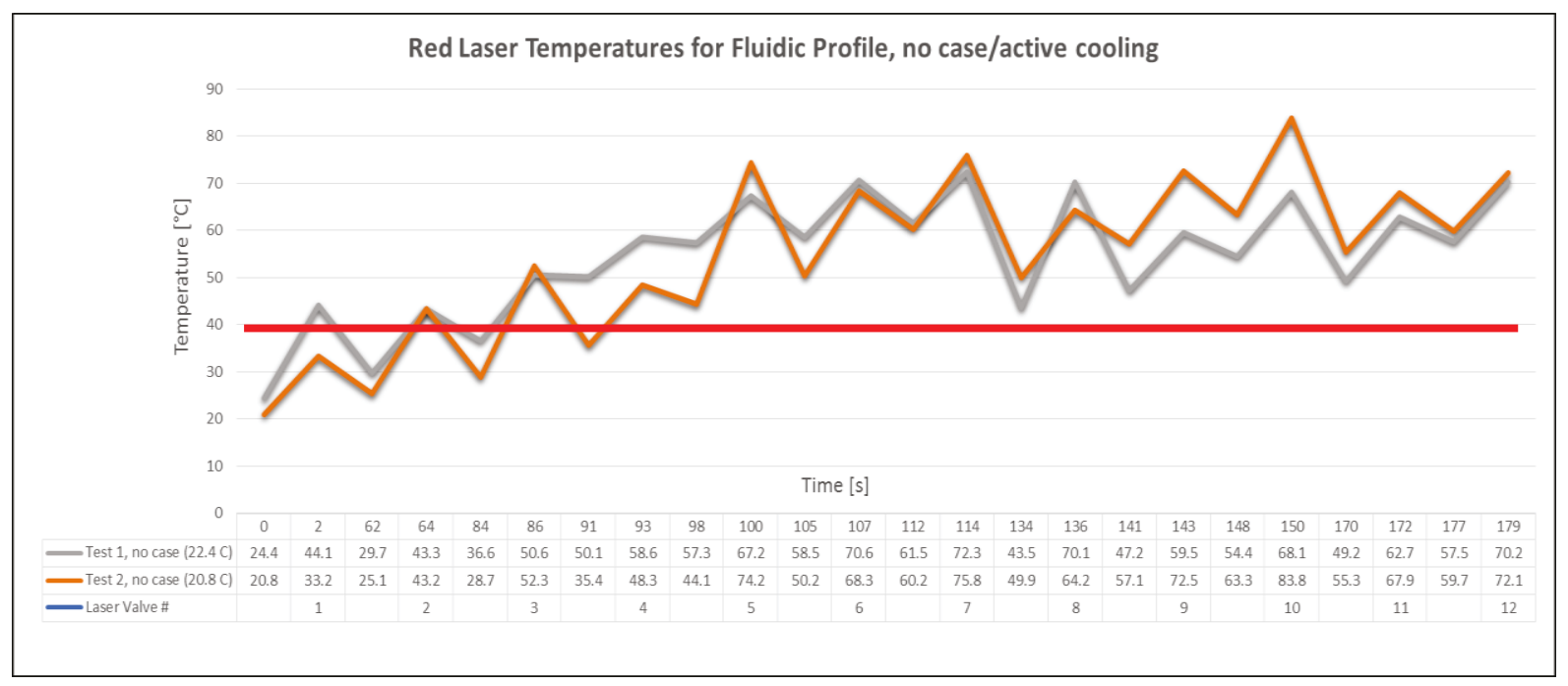

Figure 2-10. Temperature measurements over fluidic profile without cooling.

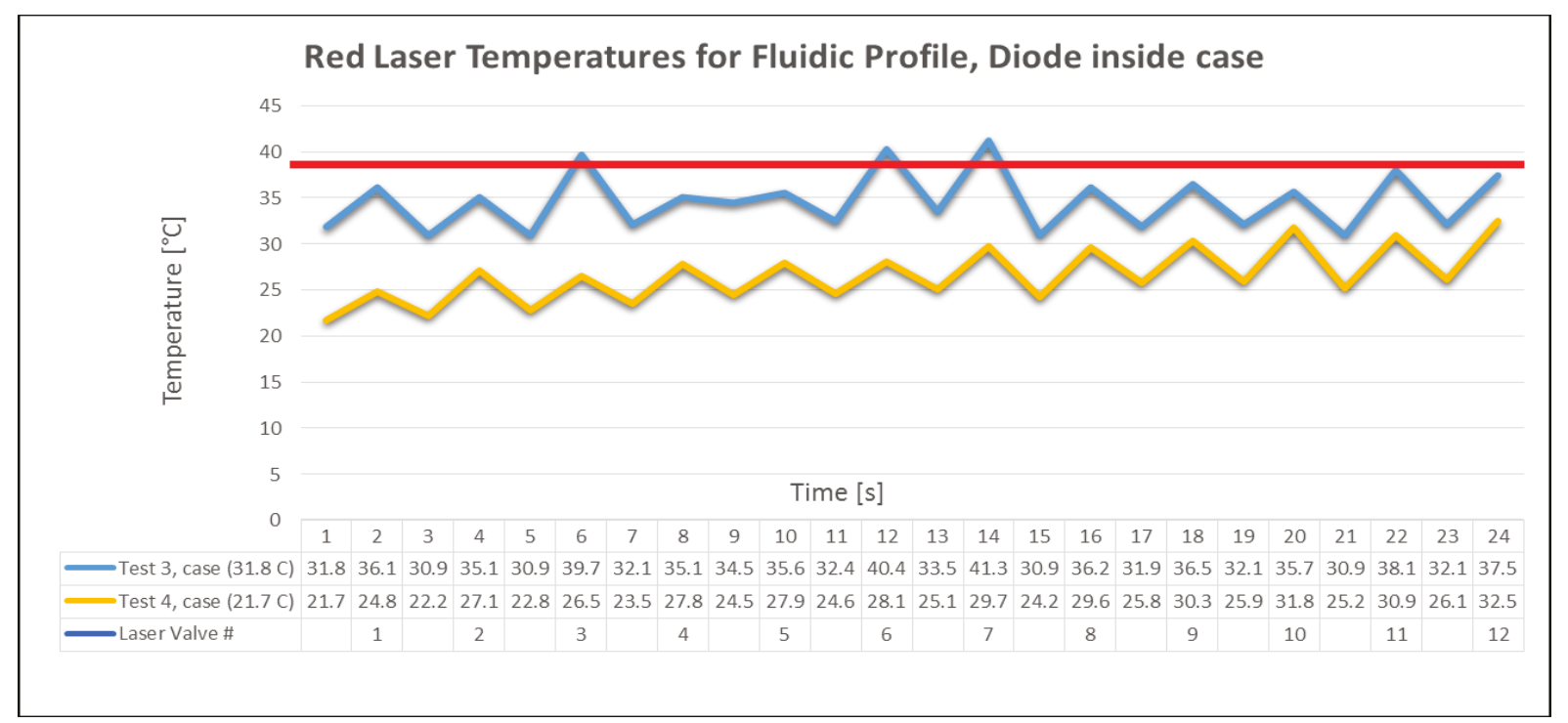

Figure 2-11. Temperature measurements over fluidic profile with cooling.

\subsubsection{Operating conclusions}

All data presented similar trends, even over different days and testing setups, suggesting the results are accurate and dependable. Given the trends, it was concluded that it was safest to use the diode inside the focusing tube and with a fan to keep air flow across the case in order to avoid ruining the lasers after only a few runs. Furthermore, adjusting the focus of the aspheric lens allowed the dwell time to decrease from $>30$ s to between $1-3$ seconds, for each 
blister pack that must be burnt through. In addition, I concluded that running the laser for less than or equal to one minutes in order to focus it wouldn't cause any undo damage as the diode would only reach about $28.5^{\circ} \mathrm{C}$, well beneath the safe operating limit.

\subsection{Detection system requirements}

In accordance with the information provided by previous projects it was known that the FASTR system would be required to detect at least three different colors at the same time, including two PCR colors and one internal lane standard to ensure peaks are being observed at the correct time. The detection range, regardless of instrument would likely need to be between $505-650$ nanometers with target sensitivity of $5^{*} 10^{-12} \mathrm{M}$ of fluorescent dye. The detector zone from the laser diode would need to be as focused as possible to maximize separation resolution in data collection; ideally the target spot size would be between $35-75$ microns.

In addition to basic system criteria, the previous short tandem repeat (STR) systems for rapid human identification also illuminated the largest potential pitfalls. The first of which is the Raman scattering peak that would need to be cut out of the detection spectrum. Raman scattering is defined by the frequency change of light due to interaction with the material the channel is made of and the energy of the vibrational modes, $v_{m}$, depends on the molecular structure and environment. The Raman signal is usually $10^{-6}$ weaker than the incident light, $\mathrm{v}_{0}$, but the photons are not absorbed and continue pinging around the system, sometimes creating "false" peaks in the detection spectrum after the "true" Rayleigh scattering peaks occur. The second major risk to the sensitivity of the detection system is high background signal. This can 
be caused by issues within the system materials as well as misalignments or undue scattering caused by the excitation laser or design flaws within the optics and filters. The detector sub assembly was the first area of focus in order to compare performance and evaluate tradeoffs between the various detection options.

\subsection{Exploring avalanche photodiodes as low-level light detectors}

In compliance with the characteristics necessary to our system we narrowed down our first choices to two avalanche photodiodes, purchased from Hamamatsu, one with a built in transimpedance amplifier (TIA) and one without. The most important evaluation criteria needed to make a decision on the photodiodes, such as quantum efficiency, dark current, noise, gain and size are summarized in Table 2-1. The equivalent noise input, ENI, is the optical power that is needed to generate a signal current that equals the noise current and was calculated using:

$$
\text { ENI }\left[\frac{W}{\sqrt{H z}}\right]=\frac{\text { Noise current }[A / \sqrt{H z}]}{M \times \text { Responsivity }[A / W]}=\frac{\sqrt{2 q I_{\text {dark }} M^{2} F}}{M \times \text { Responsivity }}
$$

The equivalent (anode) dark current input, EADCl, is the optical power that must be applied in order to produce a current equal to the dark current. In short, it is the ratio of the dark current to the responsivity. It was calculated for our photodiodes using:

$$
E A D C I[W]=\frac{M \times \text { Dark current }[A]}{M \times \text { Responsivity }[A / W]}
$$

Both of these allow us to give a more general figure of merit and can be measured experimentally and used to determine the minimum discernible signal of the detector. 


\begin{tabular}{|c|c|c|}
\hline Part Number & S5344 & S12053-10 \\
\hline Type, Dimension & Si APD, TO-5 5.9+-1 & Si APD+TIA, TO-46 \\
\hline Spectral Width [nm] & $200-1000$ & $400-1000$ \\
\hline Quantum Efficiency, M=1 [\%] & $80 @ 620 \mathrm{~nm}$ & 75 @ 550nm \\
\hline Gain & 50 & 100 \\
\hline Responsivity [A/W] & 21 & $2.7 \times 10^{\wedge} 4$ \\
\hline Dark Current, Typ/Max [nA] & $1 / 30$ & $1 / 5$ \\
\hline Excess Noise Factor & $\sim 2$ & $\sim 2$ \\
\hline EADCI [W] & $4.8 \times 10^{\wedge}-11$ & $3.0 \times 10^{\wedge}-11$ \\
\hline ENI [W/sqrHz] & $8.6 \times 10^{\wedge}-16$ & $0.1 \times 10^{\wedge}-12$ \\
\hline Active Diameter [mm] & 3.0 & 1.5 \\
\hline
\end{tabular}

\subsubsection{Current-Voltage and Gain Measurements of S12053-10 APD}

The current-voltage and gain testing consisted of a photocurrent measurement at lambda=650 $\mathrm{nm}$ and dark current measurement. The dark current measurement is obtained when a reverse-bias voltage is applied in the absence of light, creating a small detectable amount of current. The measurements were made with a probing station that utilizes probes capable of low current measurements, tens of femtoamps, and an HP 4156B parameter analyzer. Once both the photocurrent and the dark current have been measured, as seen in Fig. 2-12a, the gain of an avalanche photodiode, Fig. 2-12b, can be calculated by taking the difference of the two currents at a given voltage divided by the same quantity at unity gain, i.e. low bias voltage. From our findings the breakdown voltage of the diode was measured at $160 \mathrm{~V}$ where the curve takes off, the dark current is roughly $10^{-10}$ amperes, showing better results than expected from table above and the gain, about 100, reflects what was expected from data. 
a)

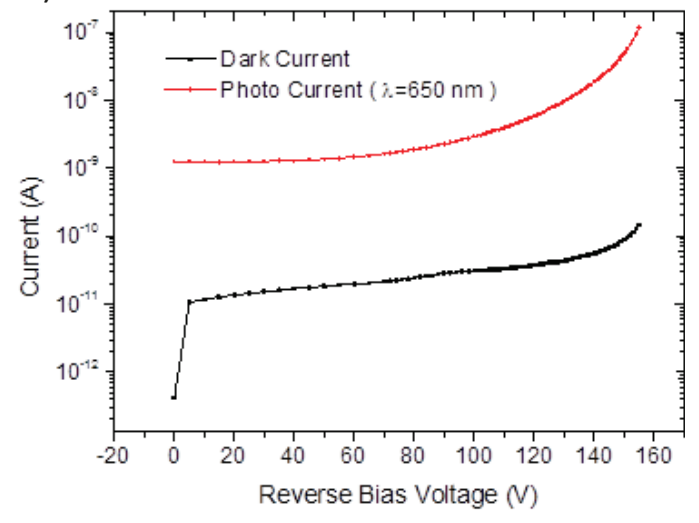

b)

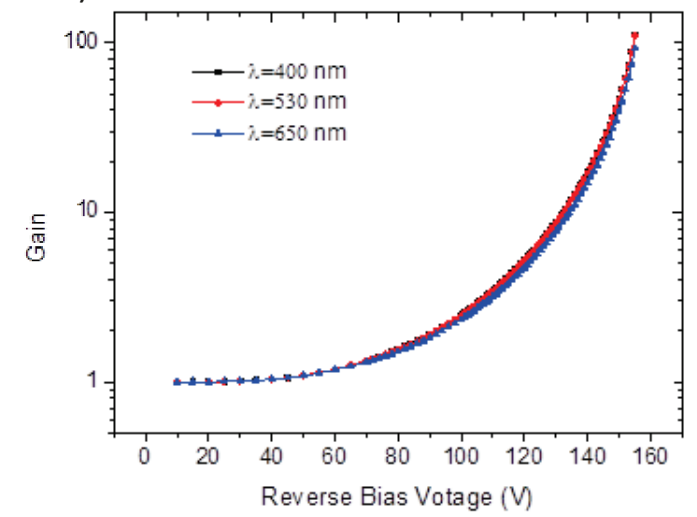

Figure 2-12. Avalanche photodiode characteristics a) I-V plot b) gain versus voltage.

\subsubsection{External Quantum Efficiency Measurements of S12053-10 APD}

As stated in background, the external quantum efficiency, as shown in Fig. 2-13a, is the ratio of the number of carriers collected to the number of photons incident on the photodiode. In order to measure this for each photodiode, a $1 \mathrm{kw}$ xenon arc lamp coupled to a monochromator functions as a tunable, narrow spectral width source spanning the spectrum from UV to the visible so that we might get a response on the diode for wavelengths starting at $400 \mathrm{~nm}$ and going up to $700 \mathrm{~nm}$ by $25 \mathrm{~nm}$ increments, shown in Fig. $2-13 \mathrm{~b}$. The output from the monochromator is coupled into a 300 micrometer core fiber which outputs the beam through two microscope objectives placed back-to-back in order to collimate the beam and then focus it into the smallest spot possible so that only the active area of the detector has signal on it, which is important for accurate measurements. The detector is then probed by two probe arms and biased using a Keithley Sourcemeter. Once this is done, a lock-in amplifier measures the photocurrent of the detector and then uses this as part of a ratio with a calibrated silicon sensor from UDT Instruments exposed to the same light in order to calculate quantum 
efficiency at a given voltage. The measured quantum efficiency for the S12053-10, shown in Fig. 2-13a, compared quite well with the given information from the data sheet showing $75 \%$ at about $550 \mathrm{~nm}$. The responsivity for S5344, shown in Fig. 2-13b, also compares perfectly with the $21 \mathrm{~A} / \mathrm{W}$ value given in the data sheets.
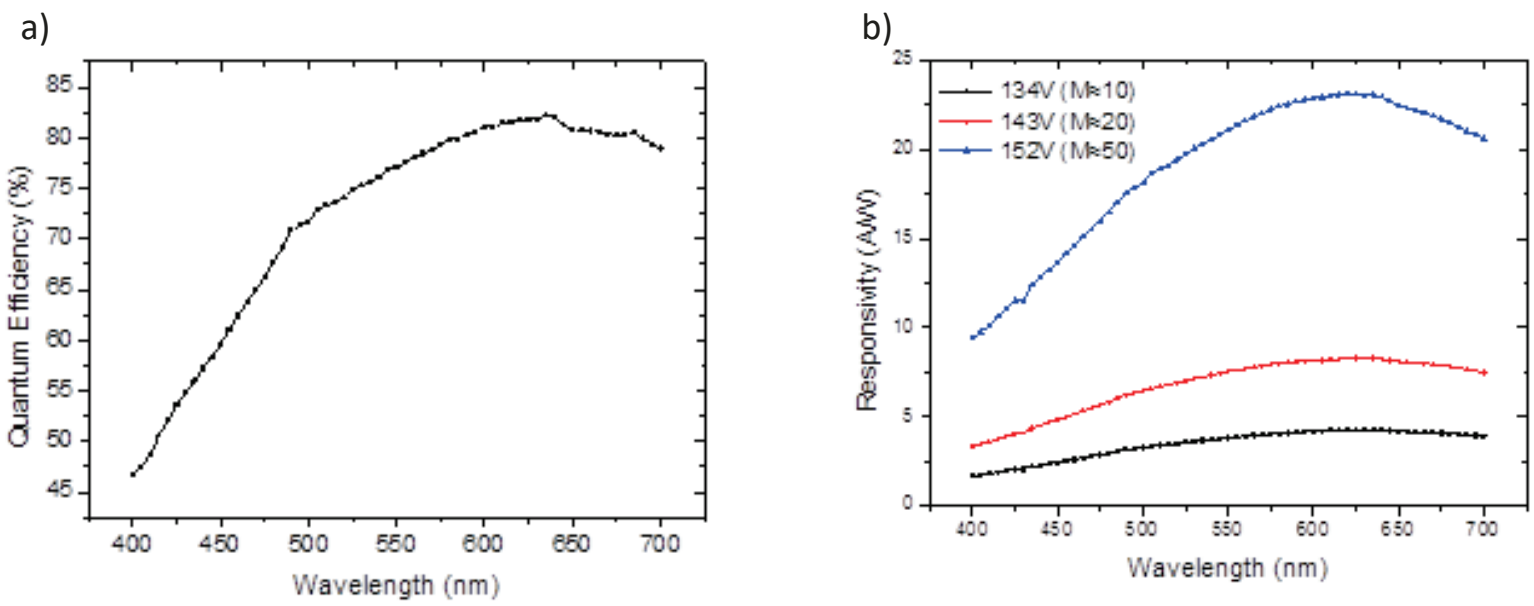

Figure 2-13. Avalanche photodiode characteristics a) quantum efficiency b) responsivity.

\subsubsection{Summary of Avalanche Photodiodes}

At first, avalanche photo diodes provide a very tempting option given how compact, cheap and easy they are to integrate into any setup. In my characterizations of the Si APD's detailed above I certainly measured a low dark current as well as low noise; however, the APD's also exhibit limited gain in comparison to the possibilities using a photomultiplier tube.

Furthermore, the signal is such that the system would still need amplification on the other side of the detector and it is difficult to say if we could decrease the dark current enough to increase our baseline detection. In testing the bench setup with fluorescein the APD's were not able to detect any lower than a concentration of $10^{-8} \mathrm{M}$ without running into the baseline and dark 
current. Given that the goals of the system are to reach sensitivities of $10^{-12} \mathrm{M}$, it seemed unwise to continue working with this device.

\subsection{Exploring single channel MicroPMT (MPMT) module as detection system}

The single channel mPMT, purchased from Hamamatsu, is comparable in performance to a standard photomultiplier tube, as shown in Table 2-2, but much more compact. Instead of a vacuum tube with multiple dynode stages creating bulk and fragility, the MPMT is designed utilizing a three-layer structure with a silicon substrate sandwiched between two glass substrates. Furthermore the anodic bonding by microelectromechanical systems, MEMS, technology that is utilized to join the silicon substrate to glass substrates makes them very shock resistant and perfect for our application i.e. rugged, high performance hand-held testing. The mPMT module consists of a mPMT, voltage divider circuit and high-voltage power supply circuit in one. The sensitivity of the module is adjusted using a separate control voltage of +0.5 through $+1.0 \mathrm{~V}$.

Table 2-2. Comparison of APD, PMT and MPMT critical characteristics given from data sheet.

\begin{tabular}{|c|c|c|c|}
\hline Part Number & H5784-01 & H12402-01 & S12053-10 \\
\hline Type & PMT & Micro-PMT & Si APD+TIA \\
\hline Spectral Width [nm] & $300-800$ & $300-850$ & $400-1000$ \\
\hline Quantum Efficiency, M=1 [\%] & $17 @ 550 \mathrm{~nm}$ & 10 @ 550nm & 75 @50nm \\
\hline Gain & $5 \times 10^{\wedge} 5$ & $5 \times 10^{\wedge} 5$ & 100 \\
\hline Responsivity [A/W] & $3 \times 10^{\wedge} 4$ & $2.1 \times 10^{\wedge} 4$ & $2.7 \times 10^{\wedge} 4$ \\
\hline Dark Current, Typ/Max [nA] & $0.4 / 4$ & $0.3 / 8$ & $1 / 5$ \\
\hline Excess Noise Factor & $\sim 1$ & $\sim 1$ & $\sim 2$ \\
\hline EADCI [W] & $1.3 \times 10^{\wedge}-14$ & $1.3 \times 10^{\wedge}-14$ & $3.0 \times 10^{\wedge}-11$ \\
\hline ENI [W/sqrHz] & $0.3 \times 10^{\wedge}-15$ & $0.3 \times 10^{\wedge}-15$ & $0.1 \times 10^{\wedge}-12$ \\
\hline Dimension & $3.7 \mathrm{in} \times 3.5 \mathrm{in}$ & $4 \mathrm{~cm} \times 2 \mathrm{~cm}$ & $\mathrm{TO}-46$ \\
\hline
\end{tabular}




\subsubsection{Initial Equipment Setup}

The initial equipment setup used to operate and test the MPMT consisted of the 488 continuous wave laser diode and controllers, three identical plano-convex lenses in their own lens holders and optical posts, one $505 \mathrm{~nm}$ long pass dichroic mirror to bend excitation beam towards sample chip, the mPMT on an xyz stage as well as the two Keithley source meters to provide the $5 \mathrm{~V}$ power supply to the $\mathrm{mPMT}$, as well as provide the control voltage $(0.5-1.0 \mathrm{~V})$ needed for sensitivity adjustments, all shown in Fig. 2-14. Each source meter was set such that neither could breach the safe operating range of voltages ruining the mPMT. In addition, the detection face of mPMT was covered with black electrical tape when not in use, in order to protect the device.

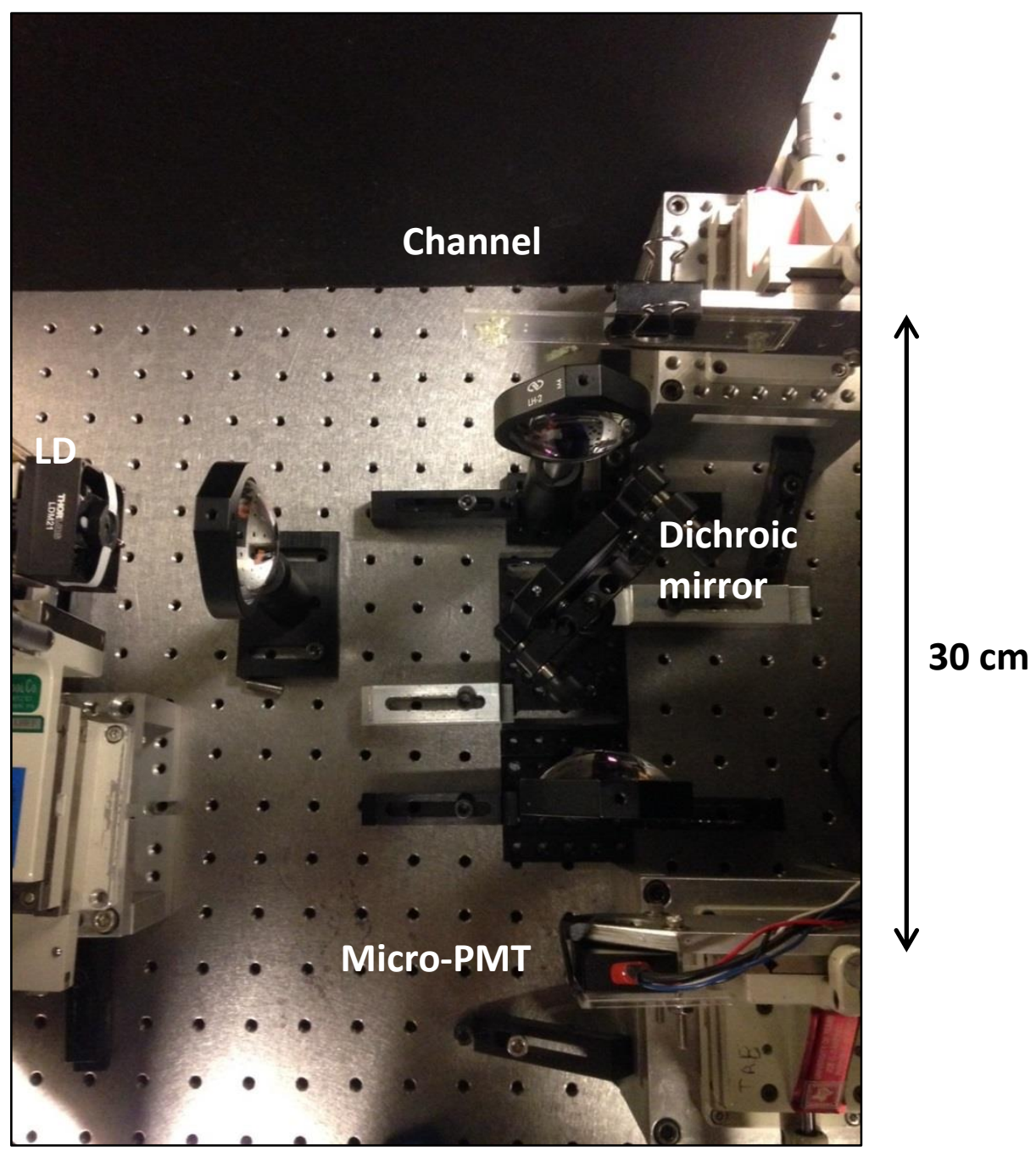

Figure 2-14. Initial equipment layout for testing $\mathrm{mPMT}$. 


\subsubsection{Testing Microfluidic Chip on Benchtop System with MicroPMT}

In order to determine how effective the MPMT actually was in our experimental setups we tested the benchtop system shown above with microfluidic chips and $10^{-8} \mathrm{M}$ concentration of fluorescein provided by the chemistry team. I was also assisted in measurements and loading and unloading chip channels with fluorescein and water by Dan Nelson.

The measurements started by using an entirely separate chip of $10^{-12}$ super bright fluor to double check that system was aligned and focused into chip as best as possible. Then we would switch to entirely separate physical chip as to avoid contamination of the fluorescein. We used this to take dark current data focused into a channel filled with water or polymer, to avoid as much scattering from chip walls as possible. We then readjusted the channel with $10^{-8} \mathrm{M}$ concentration and tested sensitivity across the entirety of control voltage $+0.5-+1.0 \mathrm{~V}$ in $0.1 \mathrm{~V}$ intervals giving data shown in Fig. 2-15. The purple line represents the signal in addition to the background currents using fluorescein $10^{-8} \mathrm{M}$ concentration. The green line represents the current output from mMPT from background with just water in chip channel. The blue line represents the dark current measured before either of the others.

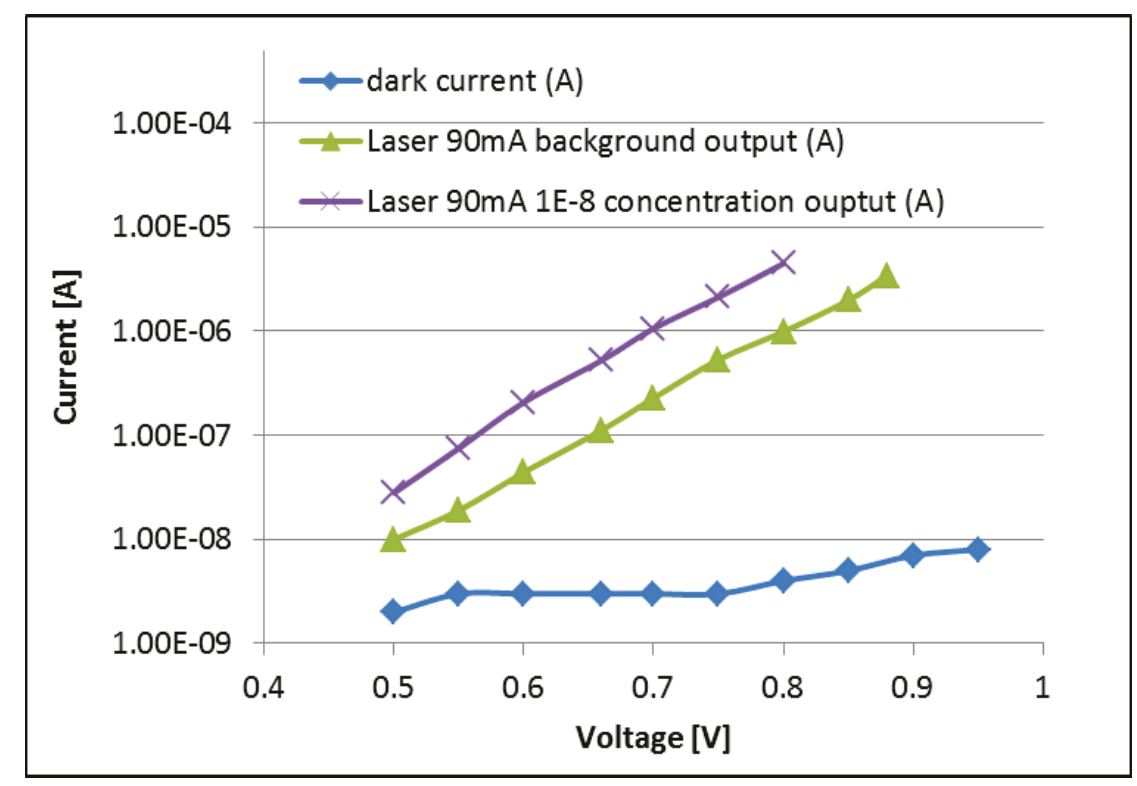

Figure 2-15. Current versus voltage characteristics of mPMT. 
The sensitivity of the MPMT changes very quickly, increasing the gain rapidly, in the large end of the control voltage from $0.8-1.0 \mathrm{~V}$. The large signal, in the micro amps, detected from our $10^{-8} \mathrm{M}$ concentration at a control voltage of $0.8 \mathrm{~V}$ is very encouraging, because there is still so much opportunity for increase. It would seem at this point that further reducing the background and stray light as well as streamlining the optical components and placing them on rails to avoid any mis-alignment would allow us to detect $10^{-12} \mathrm{M}$ at around control voltage (gain) of $0.95 \mathrm{~V}$. Given these conclusions, the components of the system are ready to transfer to optical breadboards and rails and even expanding out to the three colors expected instead of just fluorescein.

\subsubsection{First integrated setup: Multi-Color System 1}

With Dan Nelson and Tiffany Ly's assistance, all of the previously mentioned components were used to build the Multi-Color system as shown in Fig. 2-16.

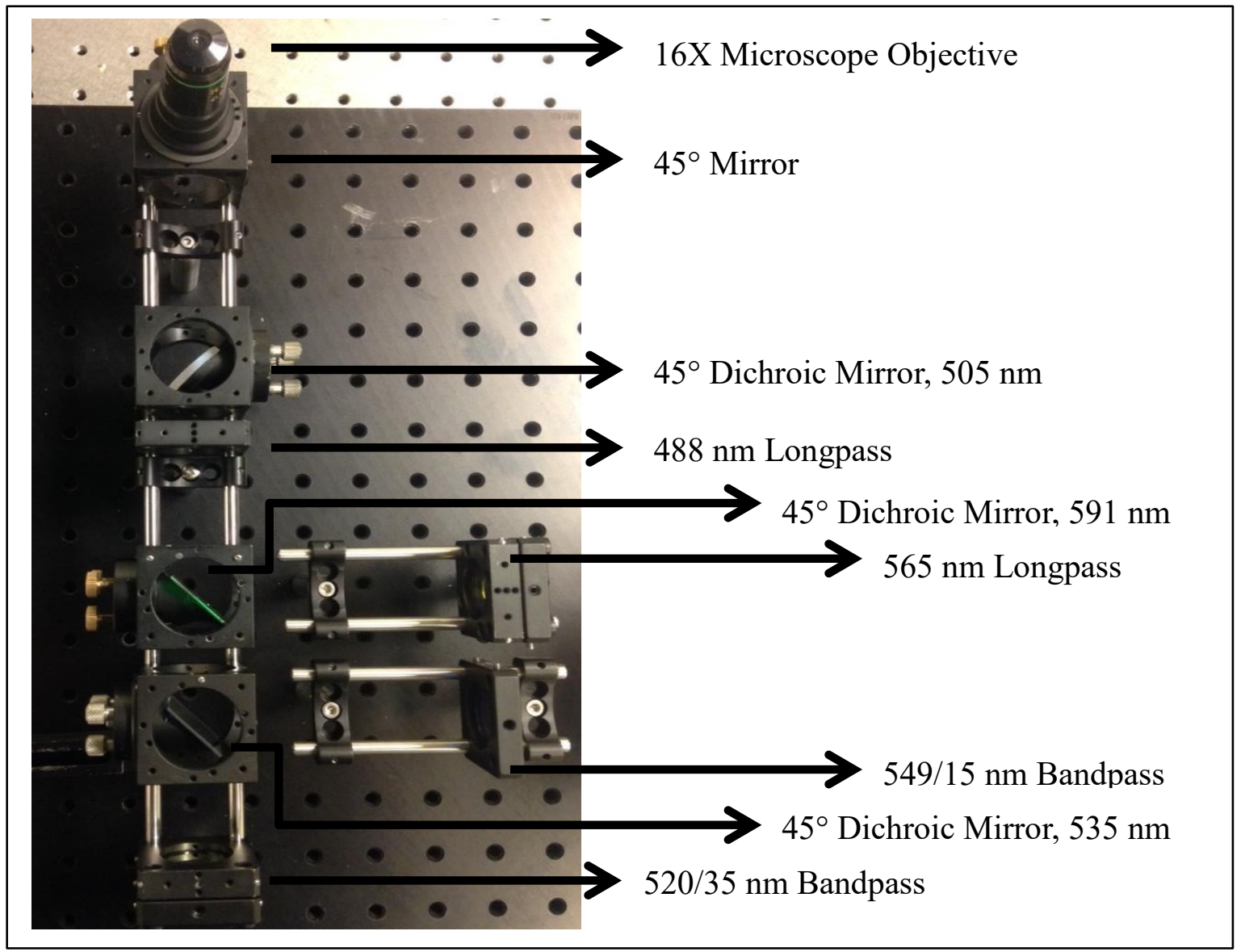

Figure 2-16. Filter, mirror and equipment layout for first multi-color system. 
In addition to the three mPMT's, held at uniform gain control voltage of $0.7 \mathrm{~V}$, laser diode and 505nm dichroic mirror, we also added a 488nm longpass filter to assist blocking any stray excitation light, $565 \mathrm{~nm}$ longpass filter to make sure nothing but ROX dye reached first mPMT, 549/15 $\mathrm{nm}$ bandpass to help shape the green signal on second mPMT and finally 520/35nm bandpass filter in front of FAM mPMT which should get only the smallest emitted wavelengths. A detailed diagram depicting the color breakdown of each fluor used is shown in Fig 2-17.

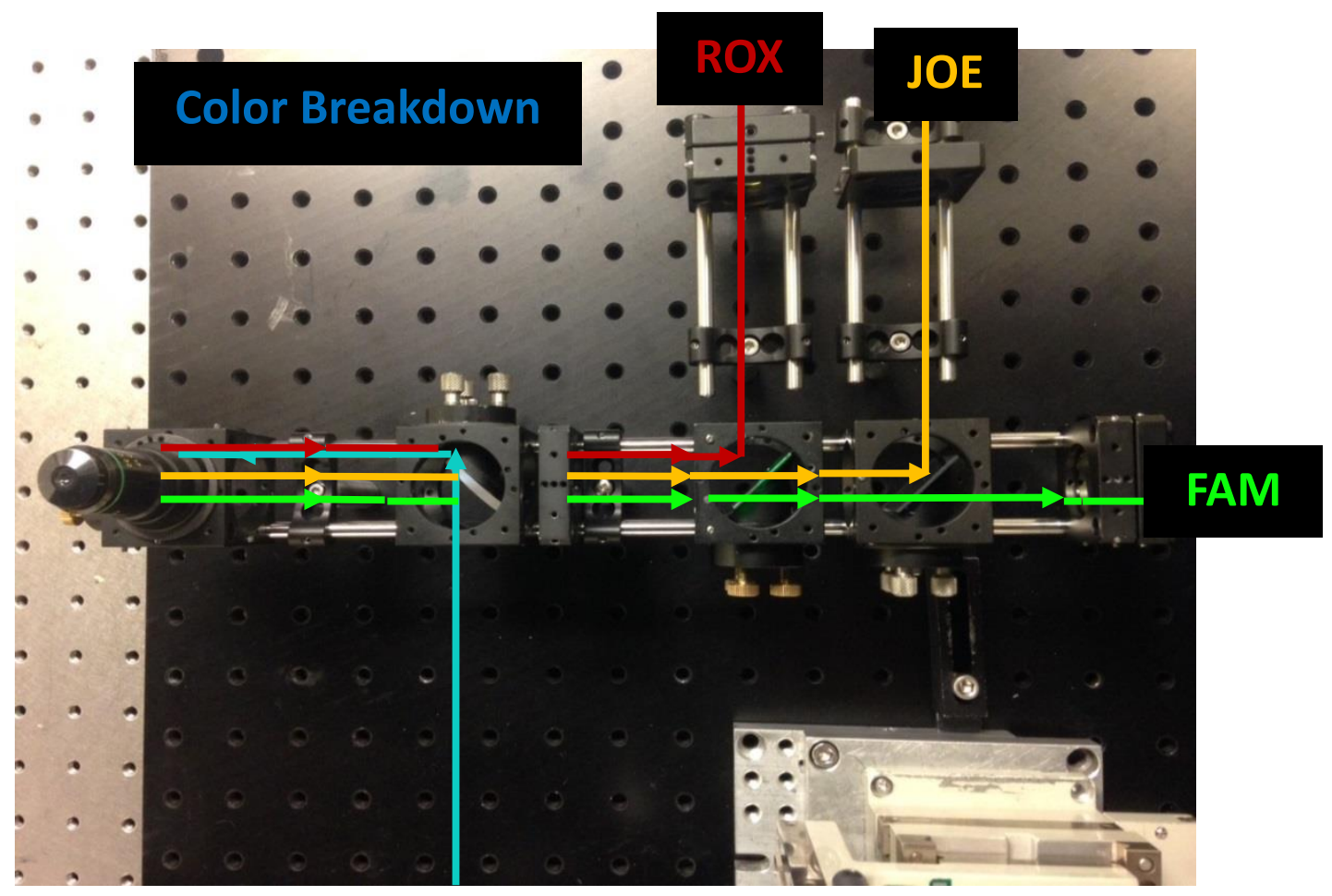

Figure 2-17. Fluor color pathway breakdown in multi-color system one.

We tested to see what colors were showing up on each detector i.e. efficiency of our filter choices in order to best decide if these were the correct choice to avoid "crosstalk" but also not cut off so much signal that we couldn't collect all of the desired sample. At this time, three 
avalanche photodiodes were installed in place of the mPMT's in order to assess with a more efficient system if there was still any chance of using the smaller, more rugged detector. The photodiodes detected the same range of colors as the microPMT, distinguishing the correct color at the correct location; however, the signal achieved on the photodiodes remained very small, in the range of $60-100 \mathrm{nA}$. From this it was decided that the microPMT's would still be the best choice for detection. Furthermore, the filters performed as expected with just a slight uptick in JOE, yellow, at both the JOE and ROX detectors. It seemed some of the signal is initially getting blocked out. Dan Nelson provided analyzed data (see Fig. 2-18) with time on the x-axis and amplitude on the $y$-axis that we received off this system using analysis software already in place. The data showed clear, distinct peaks in line with the allele ladder (ROX) in both blue and green and furthermore, the baseline seemed uniformly low.

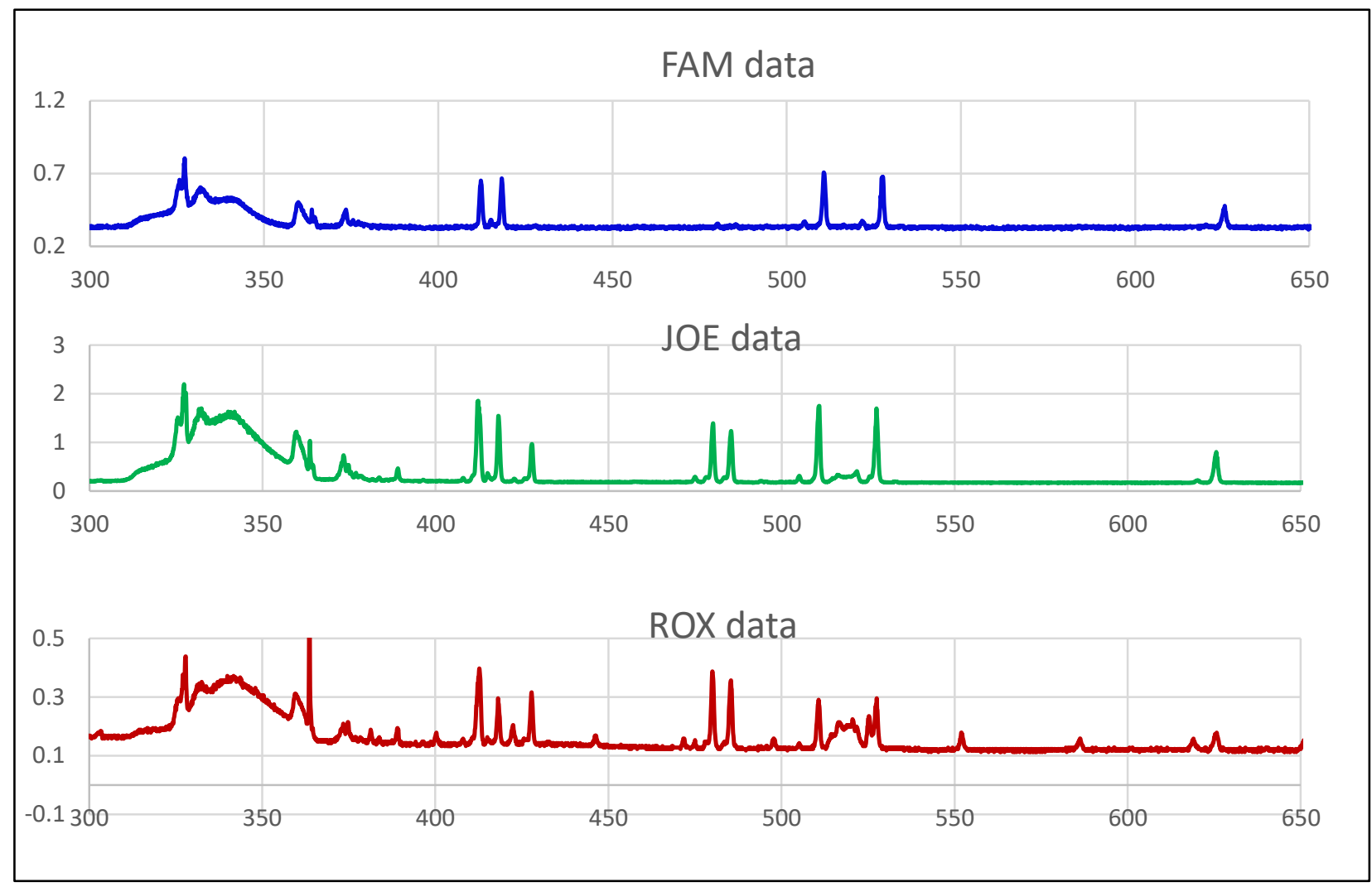

Figure 2-18. Analyzed data retrieved from mPMT on multicolor system one. 


\subsubsection{Pros and Cons of Multi-Color System}

In general the system works quite well. We achieved the high levels of sensitivity by being able to adjust the control voltage on each mPMT. This helps to adjust for the differences in excitation from the $488 \mathrm{~nm}$ laser diode in the fluors (red is the farthest from peak excitation) and this setup allowed us to decide how to shape the beams in hopes of collecting as much of the weakest signal (red) as possible while allowing the FAM to travel farthest hopefully being less likely to get interfered with by the stray $488 \mathrm{~nm}$.

On the other hand, we have now introduced quite a lot of parts and pieces. The system definitely succeeded in experimentally showing we could miniaturize the equipment but the addition of so many pieces potentially weighs down this advantage. Many free standing fragile optics i.e. dichroic and filters make the theory of adding in more colors cumbersome at best. Furthermore, our footprint would only increase as we needed to continue turning the beam and introducing more optics and more mPMT's. This certainly is not saving on cost or creating a system that can be considered rugged and compartmentalized.

\subsection{Linear array multianode PMT module as final detection system}

The linear array PMT performs nearly identical to the individual mPMT's that were being used within our Multi-Color 1 system. The details and similarities of these characterizations are shown below in Table 2-3. The new PMT is a linear array of eight channels with all the filters and optical mirrors embedded inside the module. In addition, the PMT module contains a divider circuit, pre-amplifier, high voltage power supply and gain control function so that the gain on each channel can be controlled individually with a control voltage of $+2.0 \mathrm{~V}-+4.0 \mathrm{~V}$. 
Table 2-3. Comparison of data sheet specifications for MPMT and linear array PMT.

\begin{tabular}{|c|c|c|}
\hline Part Number & H12402-01 & H9530 (-01/-20) \\
\hline Type & Micro-PMT & Linear Array PMT \\
\hline Spectral Width [nm] & $300-850$ & $300-880$ \\
\hline Quantum Efficiency, M=1 [\%] & $10 @ 550 \mathrm{~nm}$ & 20 @ 500nm \\
\hline Gain & $5 \times 10^{\wedge} 5$ & $3.0 \times 10^{\wedge} 6$ \\
\hline Responsivity [A/W] & $2.1 \times 10^{\wedge} 4$ & $2.5 \times 10^{\wedge} 4$ \\
\hline Dark Current, Typ/Max [nA] & $0.3 / 8$ & $0.5 / 8$ \\
\hline Excess Noise Factor & $\sim 1$ & $\sim 1$ \\
\hline EADCI [W] & $1.3 \times 10^{\wedge}-14$ & $2.23 \times 10^{\wedge}-14$ \\
\hline ENI [W/sqrHz] & $0.3 \times 10^{\wedge}-15$ & $0.1 \times 10^{\wedge}-15$ \\
\hline Dimension [cm] & $4 \times 2$ & $3.5 \times 1.6 \times 3.6$ \\
\hline
\end{tabular}

In order for this module to work with our current system, I worked with Daniel Mills, the mechanical engineer on our team to readjust the optics pathway and help design the apparatus that would then be 3D printed in the lab. Together we modeled just the optical pathway in AutoCad, Fig. 2-19a, and then Daniel Mills incorporated that optical model into his overall system model shown in Fig. 2-19b. The following chapter will provide more information regarding the microscope objective design; I simply wanted to show the structure and mechanics that changed between our original Multi-Color System and the current one.
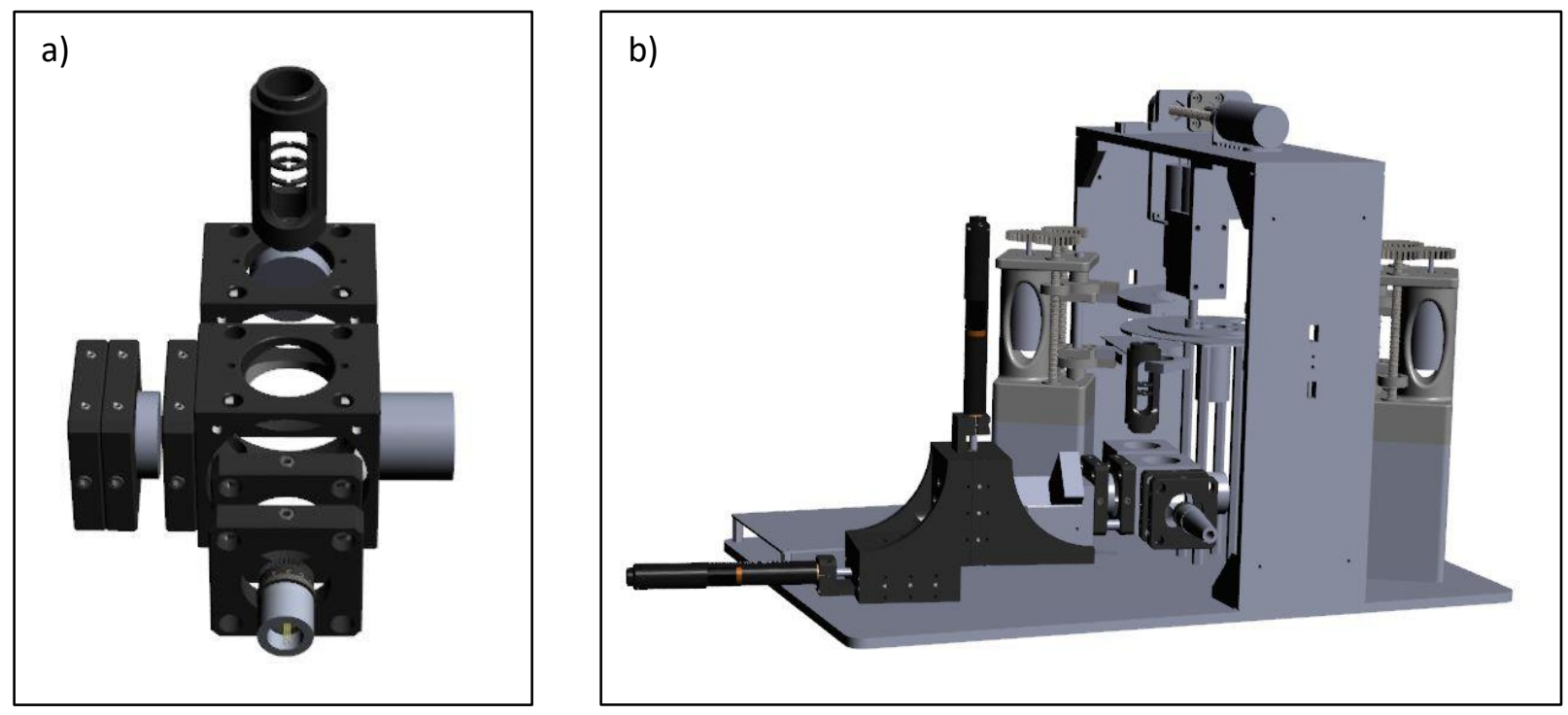

Figure 2-19. 3D Autocad designs for a) optical stack b) multicolor system two. 


\section{Chapter 3. Lensing and Integrated Systems}

In order to accurately make changes to the optics within each system I used Zemax OpticStudio to first model the ideal system and then integrate those parameters with our actual system built on optical breadboards. This chapter will discuss the basis for each modeled system beginning with the original two inch lens setup, progressing into the final miniaturized optical components. I will also discuss the steps taken to design and fabricate the objective lensing used to focus our $488 \mathrm{~nm}$ excitation laser into the chip channel and then collimate the resulting signal beam into the detection system. In the later part of this chapter I describe the final devices and optics used to build the year end prototype that met most of the goals set out in this thesis.

\subsection{First Optics Modeling: $60 \mathrm{~mm}$ Spherical Lens with Single Color mPMT}

At the start of the FASTR project, our first objectives were to establish that data could still be retrieved from a simplified table top setting, using only the 488nm laser diode on an xyz table, micro photomultiplier tube on an xyz table, 500nm spectral filter, three spherical lenses, and a longpass dichroic mirror, with all the parts being secured into an optical table. The original design for beam path and fluorescence detection alongside the actual experimental setup, depicted from a top-down point of view, is shown in Fig. 3-1a. The 488nm excitation light is outlined in blue arrows and the emission spectrum, $520 \mathrm{~nm}-620 \mathrm{~nm}$, is outlined in red arrows. The lenses $f 1, f 2$, and $f 3$ are all identical plano-convex spherical lenses with focal distances of $60 \mathrm{~mm}$ and diameter of $50 \mathrm{~mm}$. 

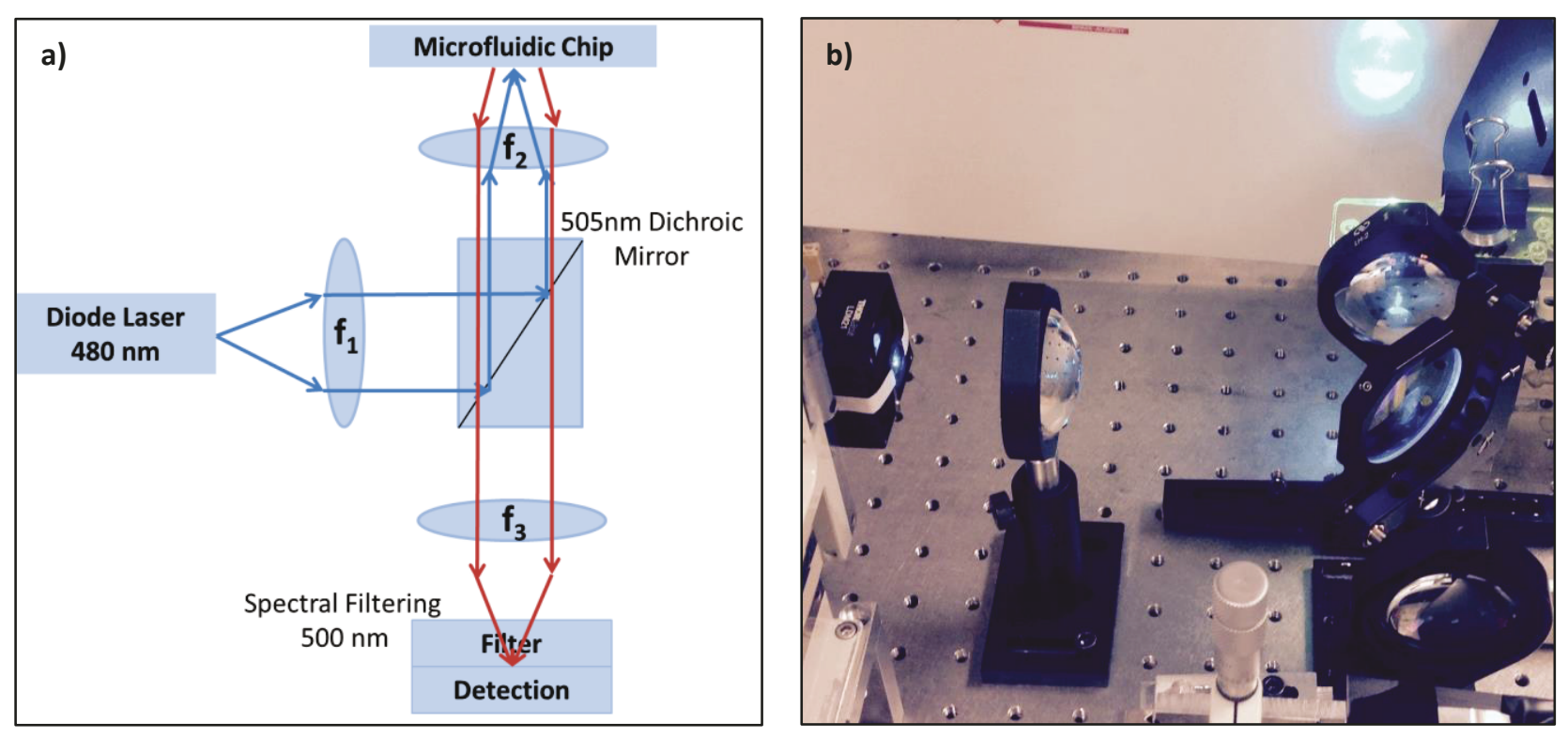

Figure 3-1. Initial optical setup with spherical lenses a) top-down view b) actual experimental setup.

Each lens was secured in its own fixed optic mount on individual optical post assemblies, spaced to match the object and image distance of each lens. The second spherical lens was used in place of a microscope objective, in order to experiment with the minimum amount of lensing needed to detect a signal. The longpass dichroic mirror is round cut with a diameter of $50 \mathrm{~mm}$ in order to match the spherical lenses and catch as much of the excitation beam as possible. The dichroic has a cutoff wavelength of $505 \mathrm{~nm}$, with a reported $85 \%$ absolute transmission band of $520 \mathrm{~nm}-700 \mathrm{~nm}$ and $90 \%$ absolute reflection band of $380 \mathrm{~nm}-490 \mathrm{~nm}$. The mirror was oriented such that the reflection band would turn the excitation beam path $90^{\circ}$ towards the microfluidic chip and the transmission bands would allow the entirety of the emission spectrum to pass back through the mirror towards the detector. This orientation was chosen in order to eliminate the need to turn the fluorescent emission beam, detecting as much of the resulting energy as possible. In addition, placing the laser diode at a 90 degree angle to our detection path (see Fig. 3-1b) increases the odds that stray light will not reach the 
detection face. Finally, a 505nm long pass spectral filter, one that would not allow any wavelength below $505 \mathrm{~nm}$ to pass through, was placed in front of the detection face in order to block any stray, or reflected, light from the laser diode.

\subsubsection{Analyzing Spherical Lenses}

For the first simplified setup, spherical lenses were chosen because regardless of what type of beam we have, parallel or dispersive, one side of the spherical lens could be used to achieve the opposite ray formation ${ }^{7}$. In this sense, spherical lenses provided the broadest, most robust capabilities without needing to know exactly what our system required in order to begin testing. The spherical lenses were placed at an object distance of $60 \mathrm{~mm}$, the given distance to either focus rays of light into a point, or create a parallel, mostly collimated beam depending on direction of travel. The ray diagram of our single spherical lens is modeled in Fig. 3-2, illustrating how each marginal ray crosses at a slightly different time creating the minimum blur point at about $60 \mathrm{~mm}$ away from the rear face of the lens. Each lens performed exactly as expected; however, it became very clear that placing the first lens $60 \mathrm{~mm}$ away from the laser diode would not provide adequate collection of the laser beam, as the diode is expanding too fast. Furthermore, placing the second lens $60 \mathrm{~mm}$ away from the microfluidic chip would not efficiently collect the emission spectra, giving too much space for the cone of emission to miss our lens entirely, never making it to the detector.

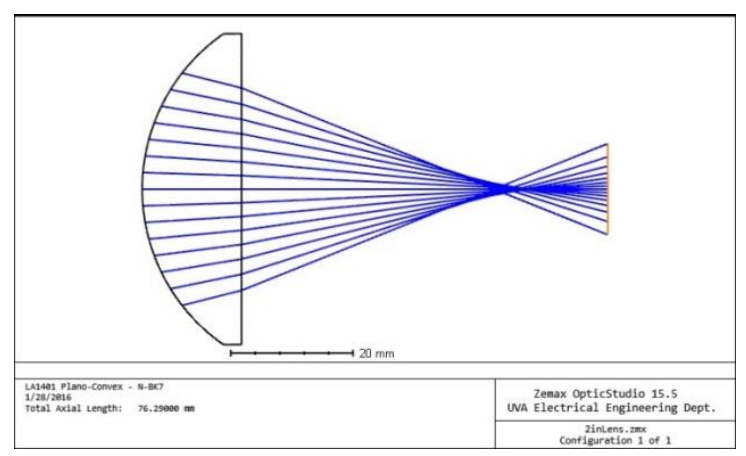

Figure 3-2. Model of single spherical lens with a collimated incident beam. 


\subsubsection{Microfluidic Chip Focus}

At this step, there was no equipment in front of the laser diode and in order to reduce the amount of scattered light and continue testing, an adjustable pinhole was secured to the front of the laser diode mount. This was utilized as a short term solution in order to try focusing into the microfluidic chip, given that as I decreased the radius of the laser diode beam, I would also be decreasing the amount of overall power in the system.

With this adjustment, I placed a white screen behind the chip in order to get a visual image to follow while trying to focus. Initially, looking at the channel before attempting to focus, it appears as a lit circular area with an empty line through the center as in Fig.3-3a. The empty line shown is the channel of the chip. As we try to zoom in the line begins to bulge out and eventually the best focus that could be achieved with this lensing system is shown in section b. It's clear that the focal point is too large for our 80 micron channel due to the bands of light still showing around the edges. Section c shows what can be seen when the incident beam runs across the wall of a channel; a flaring of light along the top of the area tells us that we are hitting the bottom wall. If we zoom in too far and begin to see the "double-lobe" imaged in section d, it means that we have gone too far and are actually reflecting off the bottom of the chip.

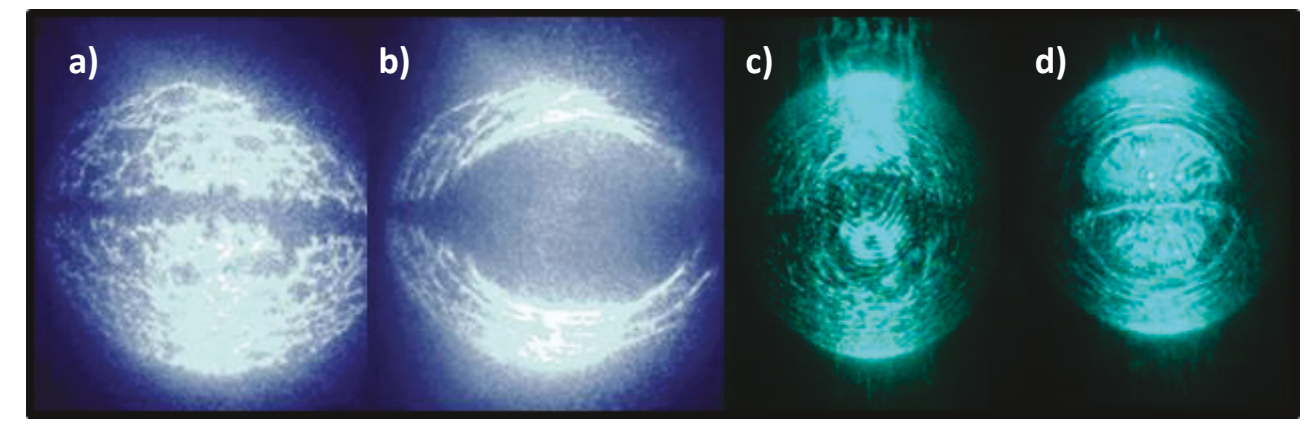

Figure 3-3. Focusing laser into microfluidic chip a) no zoom b) zoomed and center c) reflecting off bottom wall d) double lobe image. 
Ultimately the reflection on the white screen as I adjusted the focus into the channel proved that the $60 \mathrm{~mm}$ spherical lenses were unable to create a focal point small enough to fit within the chip. The corresponding model for focal point spot size and shape shown in Fig.3-4, confirmed that we would see rings of intensity within a spot size about 1200 microns in diameter.

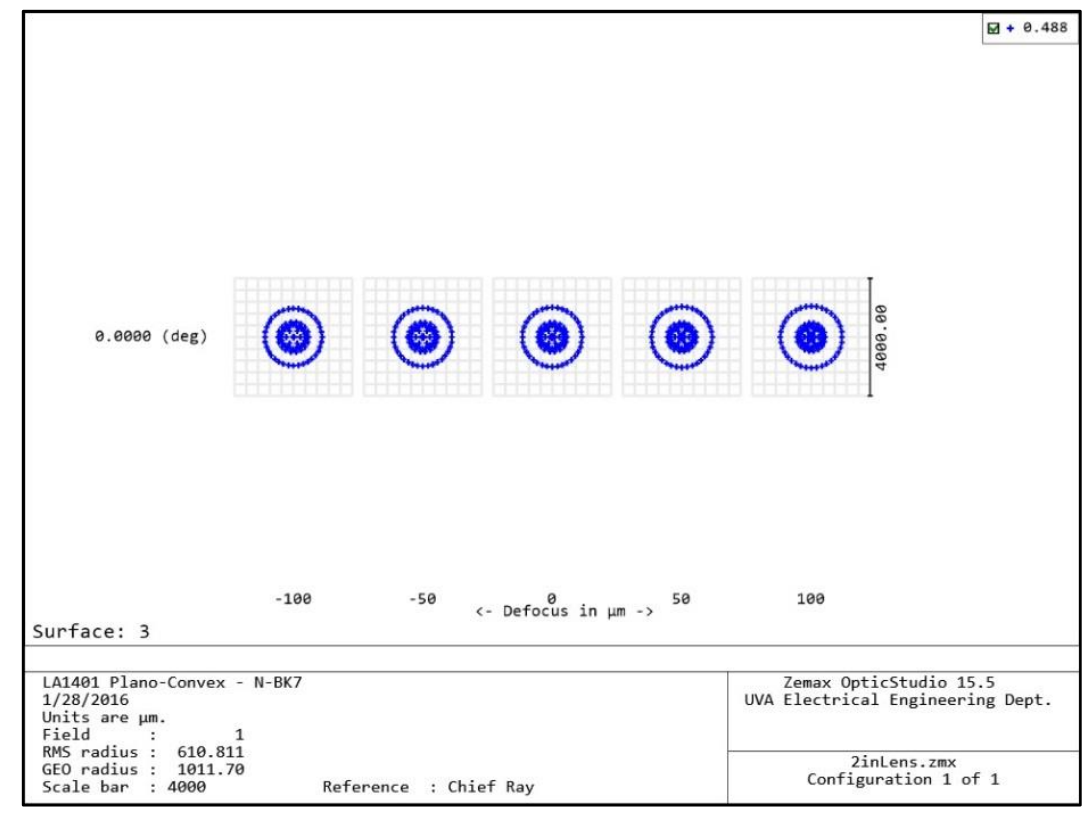

Figure 3-4. Model of spot size and shape for spherical lens.

\subsubsection{Summary of $60 \mathrm{~mm}$ Spherical Lens System}

The $60 \mathrm{~mm}$ spherical lens worked perfectly in early experiments, allowing us to measure values for the laser diode and micro photomultiplier tube characteristics. With this information, we were able to conclude that the microPMT could detect concentrations of fluorescein as low as $10^{-8} \mathrm{M}$, even with the non-efficient lensing. It was strongly theorized that improving the optical components of the system would greatly increase the signal detection levels. It also 
shed clarity on small changes to the system that would greatly increase performance, including an aspheric lens in front of the laser diode, mounting all optical components on optical cage and rail systems to ensure proper alignment, and decreasing the size of the lenses in order to decrease path length. All of this data gave us confidence to extend the system out to three colors with three corresponding micro photomultiplier tubes.

\subsection{Second Optics Modeling: Multi-Color System 1}

The first multi-color system, fully described in 2.6.3, incorporated all of the adjustments mentioned in the previous section. Each optical component was placed inside its corresponding holder and placed on a breadboard using optical rails and supports. In essence, much of the old system was maintained; we simply added a spherical lens to the front of the $488 \mathrm{~nm}$ laser diode and expanded the number of colors that can be detected at once, including all the necessary filters and mirrors to make that possible. The spherical lenses, filters and mirrors were all $25 \mathrm{~mm}$ now, instead of $50 \mathrm{~mm}$, and the footprint of the whole system fit onto a $12 \times 12$ in optical breadboard. In place of the spherical lens that previously focused our excitation beam onto the chip, is a 45 degree mirror pointing up into a $16 \mathrm{X}$ microscope objective. This microscope objective paired with the aspherical lens placed in front of the laser diode to ensure it traveled as collimated as possible drastically increased our ability to distinguish colors and reduce the background noise by fitting within the channel. All early objective adjustments and experiments were performed on the multi-color system one. 


\subsubsection{Optical filter crosstalk}

In order to purchase the correct optical filters, we needed to place the spectrum of each fluor: FAM, JOE and ROX onto the same transmission versus lambda chart as shown in Fig. 3-5. Plotting all three spectrums together allowed us to analyze how much each filter would cut into the neighboring color spectra. After giving a great deal of thought to how much signal from each fluor we could stand on each detection face, not to mention how we planned to physically lay out the color paths of the system, shown in Fig. 3-6, all of the optical filters and dichroic mirrors were purchased from Semrock, with a diameter of $25 \mathrm{~mm}$ to match the rest of the optical components.

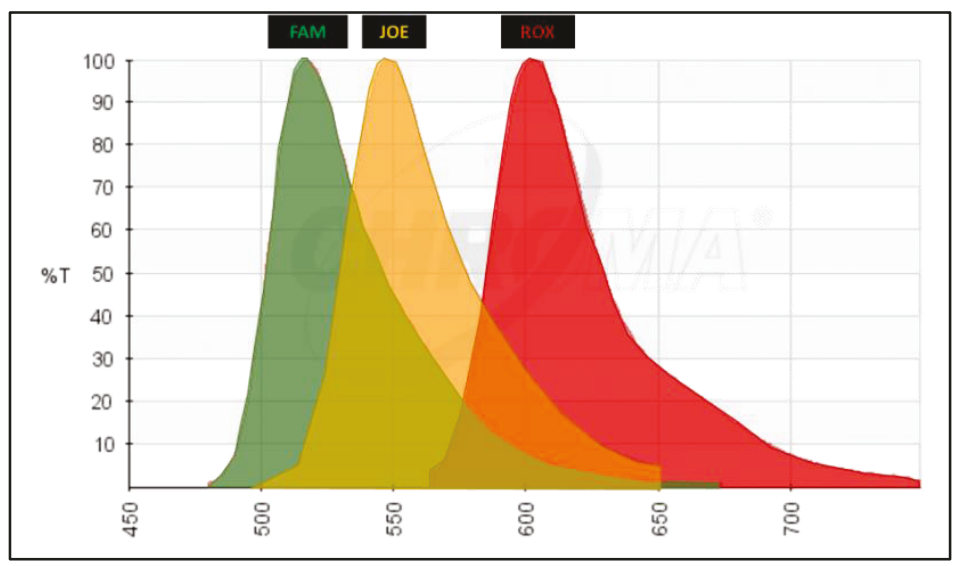

Figure 3-5. Optical transmission of FAM, JOE, ROX fluors versus wavelength.

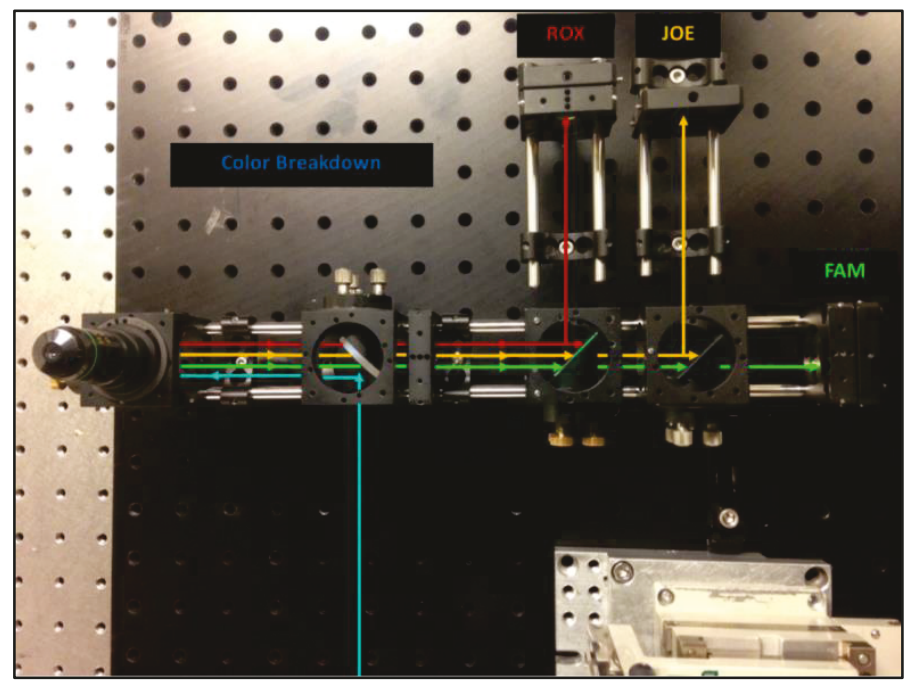

Figure 3-6. Fluor color pathway breakdown in multi-color system one. 
As can be seen from Fig. 3-6, the fluor we have to be most careful with is JOE, given that its spectrum overlaps quite a lot with both FAM and ROX. We placed a sample of each fluor separately into the channel and then measured the signal strength of that fluor, in $\mathrm{nA}$, on each detection face, shown in Fig. 3-7. The filter placed on our FAM detector was specified as a $520 \mathrm{~nm}$ band pass filter $\pm 35 \mathrm{~nm}$, and it created the cleanest signal, allowing very little JOE and a nonexistent amount of ROX. The filter placed on JOE was specified as a $549 \mathrm{~nm}$ band pass filter $\pm 15 \mathrm{~nm}$, and while there was some bleeding of FAM onto the JOE detector, this is a signal we needed to accept in order to get a strong enough signal out of JOE. Finally, after running the experiment we realized that the $565 \mathrm{~nm}$ long pass filter placed in front of the ROX detector was superfluous as our initial dichroic mirror bending ROX signal towards that detector was a $591 \mathrm{~nm}$ longpass, reflecting anything below 591 nm already. The filters all appeared to perform as expected and the overlap in signal will need to be dealt with using software later, as shifting them around could potentially cut off too much of the desired signal.

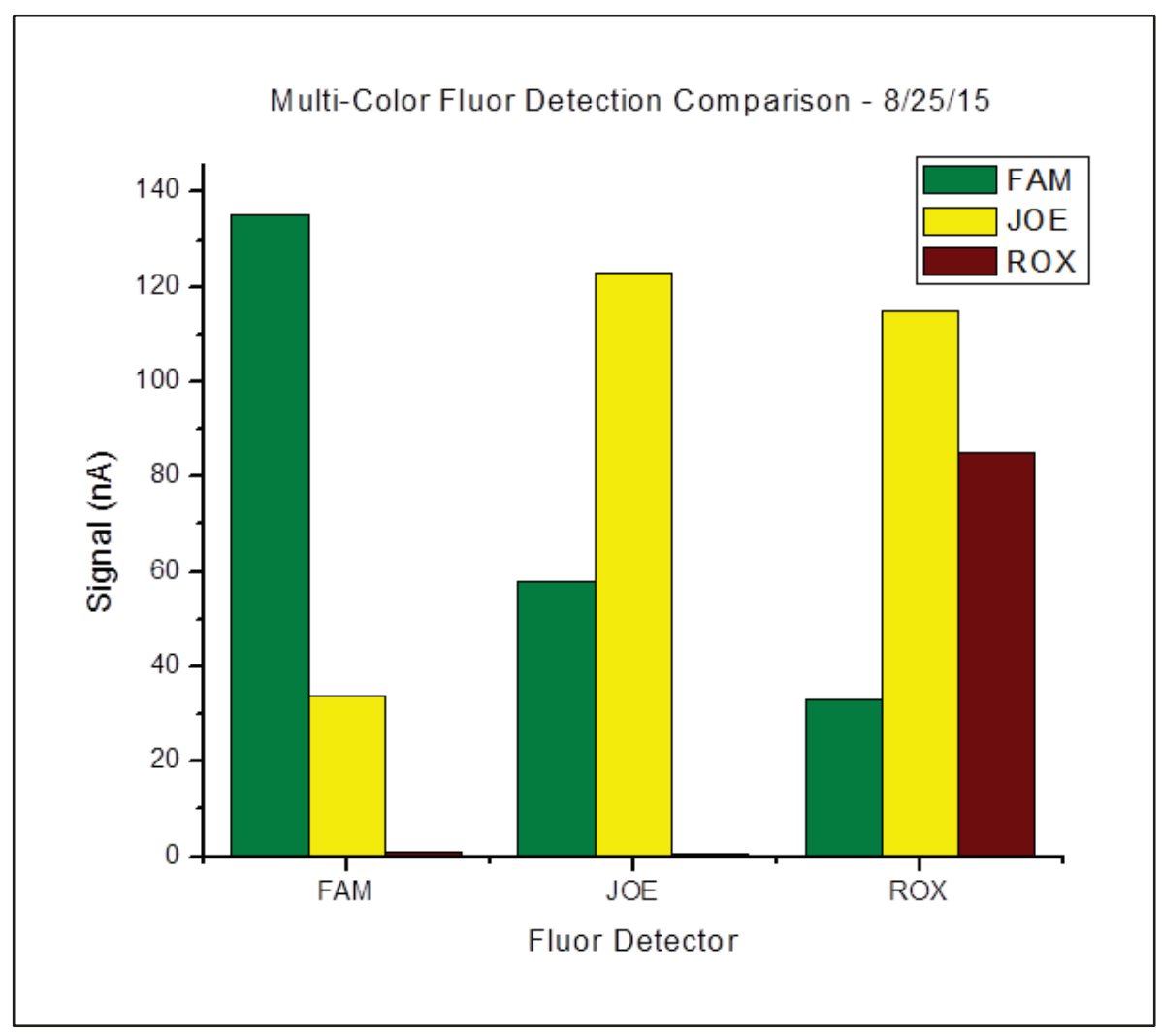

Figure 3-7. Plot of fluor signal on each mPMT. 


\subsection{Third optics modeling: integrating and minimizing multi-color system 1}

In each of the previous optical variations the laser diode emission beam was collimated but not circular. The lenses have served to collect and create a parallel beam out of the elliptical shape, but nothing has been introduced to condition the lens into a purely circular form. This particular characteristic was low in priority because we believed that a slightly elliptical beam wouldn't damage the sample as long as the length of the ellipse was aligned parallel with the channel, versus perpendicular to the channel as to avoid reflecting light from scattering off of the walls of the chip.

Many traditional solid state lasers and even laser pointers use two cylindrical lenses in succession to magnify the minor axis, $\theta_{\|}$, to better match the major axis, $\theta_{\perp}$. The $488 \mathrm{~nm}$ laser diode has a $\theta_{\|}$of $7^{\circ}$ and $\theta_{\perp}$ of $23^{\circ}$, so in order to give the diode a circular beam, the theta minor needs to be magnified about three times giving a beam shape of $21^{\circ}$ by $23^{\circ}$. The equation for magnification is

$$
M=\frac{f_{2}}{f_{1}}
$$

with $f_{1}$ representing the focal length of the first lens and $f_{2}$ representing the focal length of the second lens ${ }^{7}$. It follows that to achieve a magnification of three, our second lens will need to have a focal length three times larger than that of the first lens; however, outside of that constraint, we can choose any two focal length values. 


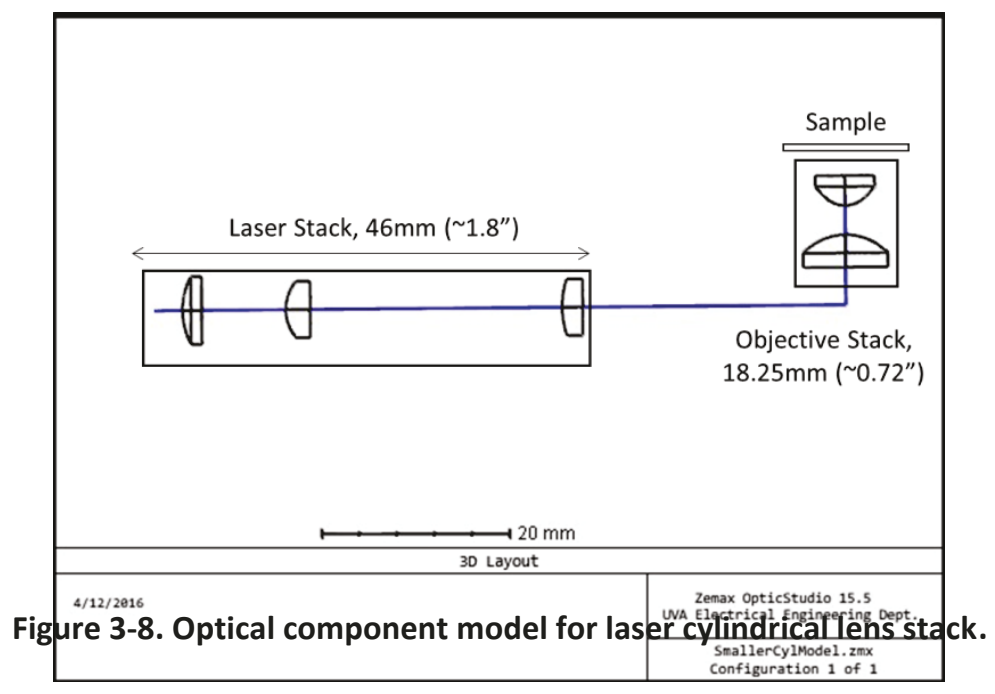

I first used Zemax to model the laser diode, aspheric lens and two cylindrical lenses, shown in Fig. 3-8, to figure out if they would actually affect the beam shape. The focal length and lens type listed from the left is $11 \mathrm{~mm}$ aspheric lens, $6 \mathrm{~mm}$ cylindrical lens, $18 \mathrm{~mm}$ cylindrical lens, dichroic mirror positioned beneath the objective stack (not shown), and the objective stack itself. The actual length of the optical laser stack is $46 \mathrm{~mm}$ and the theorized height of the objective stack is $18.25 \mathrm{~mm}$. The optics footprint, the smallest it's ever been, is about one foot from the back edge of the laser diode casing to the far right edge of the objective stack; in fact, minimizing the path length is only limited by the current mounting apparatus as many of the optics pieces could sit much physically closer. The objective and mirror stack is about six inches tall; reducing that z-height will be a top priority in designing our own microscope objective.

In addition to creating a ray diagram model of the cylindrical lens design, I took spot diagram slices just after the second cylindrical lens (see Fig. 3-9a) and at the chip (see Fig. 3-9b). The spot diagram in Fig. 3-9a, with a spot size of 84 microns in diameter, is not as circular as I would have expected, though the beam is not as severe an ellipse as it was prior to the cylindrical lenses. The spot diagram in Fig. 3-9b, with a spot size of 28 microns in diameter, taken just after the objective at the chip, shows a tightly bound elliptical shape, again, not as 
severe as the laser diode without any adjustments but not nearly circular enough to suggest adding more lenses to our system is beneficial. In practice, the cylindrical lenses were incredibly difficult to mount and required extreme finesse to align properly. Even still, any small nudge on the breadboard and the cylindrical lenses would shift within their mounts just enough that their striations wouldn't be lined up properly. Ultimately I decided that the slight adjustment achieved in the diode beam shape and spot size did not outweigh the high risk that the cylindrical lenses would not be rugged enough to last any rough handling of the device.
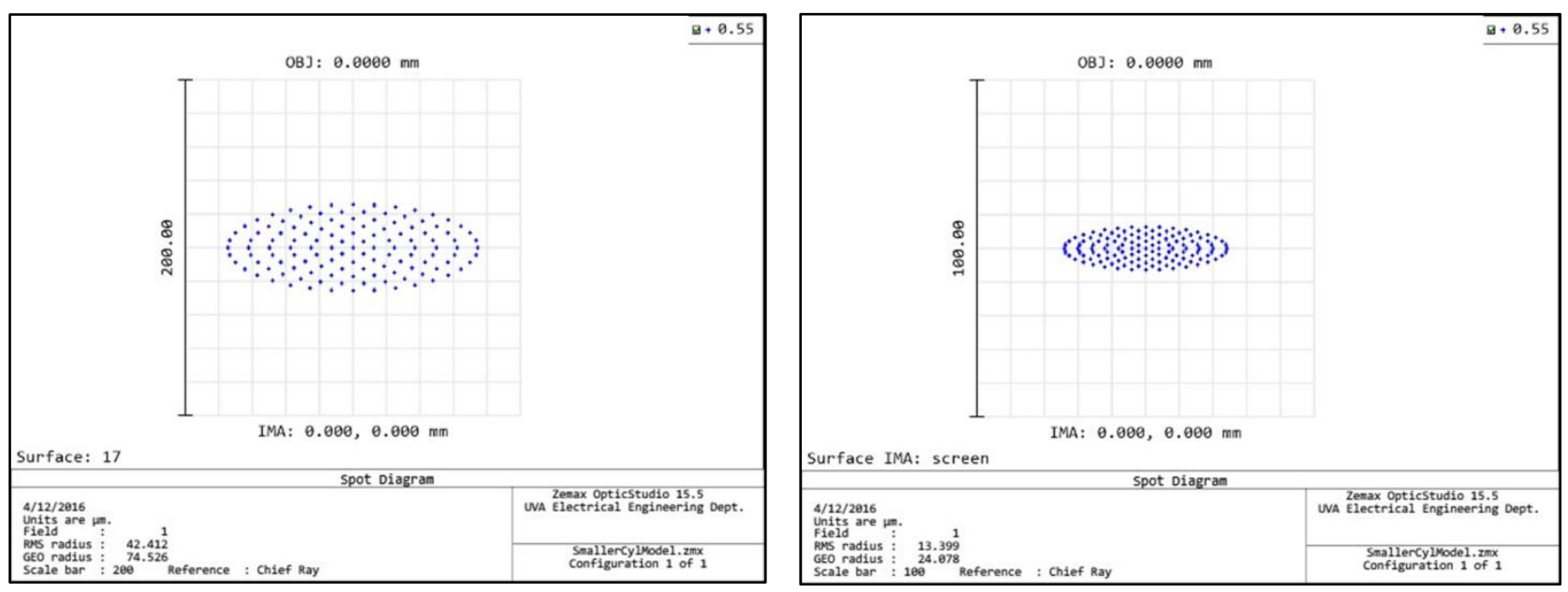

Figure 3-9. Spot diagram size and shape a) just after second cylindrical lens b) at chip.

\subsubsection{Laser Placement and Focus}

In response to ruling out the use of cylindrical lenses to adjust the laser diode beam shape and size, I decided to test my microscope objective designs using just the aspheric lens mounted in the front of the collimation tube. When the aspheric lens was originally placed in the system, it simply screwed in and was secured at its default position. However, the front of the collimation tube allows for a $2.5 \mathrm{~mm}$ range of adjustment in the laser's focal point. This 
range, shown in Fig. 3-10, shows a collimated beam uniform on the reflecting mirror for $\mathrm{f}=1.8 \mathrm{~mm}$, point at which lens is closest to diode face, and then as the focal length increases, the focused point of the laser shifts left, back towards the laser diode as the aspheric lens moves farther from the diode face. Given this information, the beam and size of the laser diode light will be adjustable without needing to introduce additional lenses.
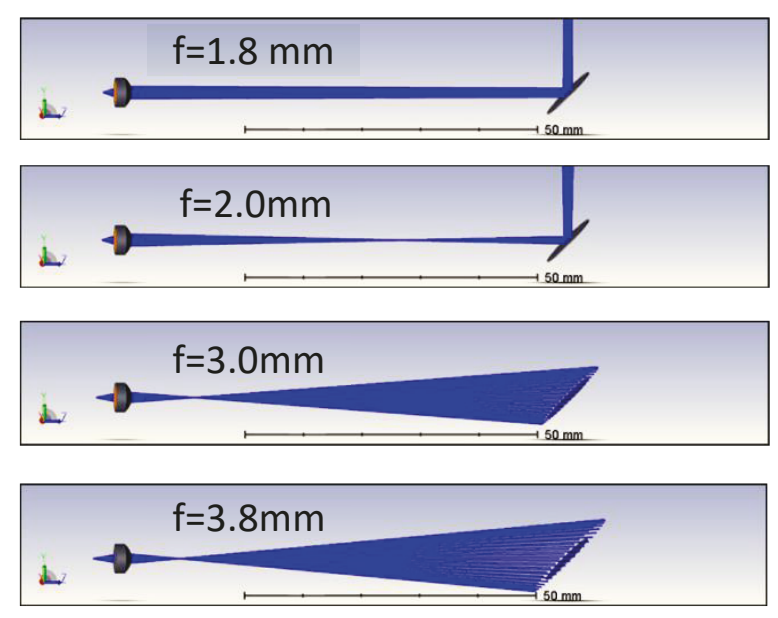

Figure 3-10. Laser focus adjustments from $1.8 \mathrm{~mm}$ to $3.8 \mathrm{~mm}$.

\subsection{Fundamental microscope objective structure and design criteria}

Microscope objectives are one of the most important, complex components in any microscope as they are responsible for primary, real, image formation. They determine the magnification of a particular sample and the resolution under which fine details can be observed. The most relevant information such as manufacturer, numerical aperture, magnification color band etc., is always printed right on the objective barrel ${ }^{2}$. A compound microscope is one that contains multiple lens elements including various lens types, lens 


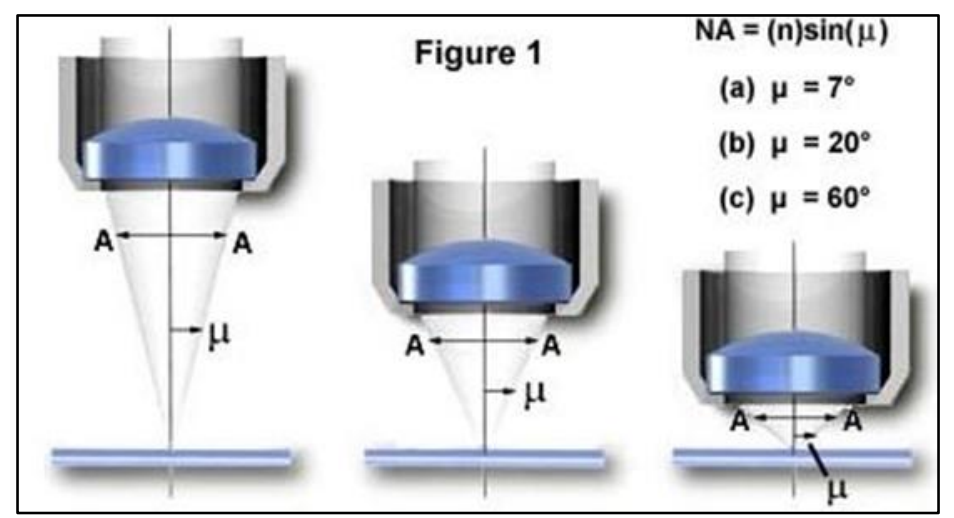

Figure 3-11. Numerical aperture versus working distance of microscope objective. ${ }^{2}$

doublets or triplets, spacers and aperture rings. The achromatic is the most simple and common of all the objective types. They usually contain two lens doublets and a front meniscus lens capable of correcting chromatic aberration for red and blue and spherical aberration for green ${ }^{2}$. This type does not correct for field curvature and if this was truly a problem, the system would need to upgrade to a plan achromat which is much more complicated and expensive. The most critical design characteristics which ultimately set the resolution limit are the wavelength of light used to illuminate the specimen, refractive index in object space and the angular aperture of the light cone captured by the objective, expressed as $N A=$ $n \sin \left(\theta_{\max }\right)$ and illustrated in Fig. 3-11, using $\mu$ instead of $\Theta$. The numerical aperture of any objective is a function of the focal length and the entrance pupil diameter, which determines how much light, enters the first lens. A larger angle, and numerical aperture, will correspond to a greater amount of light entering the objective.

In relation to the FaSTR project, and most florescent spectroscopy systems, the working distance, or distance from the front of the objective to the chip, is also very important and will ideally be a distance ranging in the microns. The fluorescing spectrum emits as a cloud in every 
random direction; so, the closer the objective rests to the chip, the higher the chance that the objective collects as much of the resulting energy as possible.

The goals for creating our own microscope objective center on minimizing the number of optical components, while maintaining an ideal numerical aperture and working distance. We would also like to integrate the $45^{\circ}$ angle mirror underneath the objective into the objective stack itself using a lens tube and in-house designed apertures, made from multiple layers of black PET paper. The final goal is to shrink the height of the multi-color system 1 objective stack from six inches to about two inches.

\subsubsection{Modeling disassembled microscope objective}

The design of microscope objectives is a heavily guarded topic with very little information available to assist making one on your own. In order to get a better understanding of the blueprint for a simple achromatic objective, I dissembled an extra one from our lab, measuring as much information as I could. The objective had a numerical aperture of 0.32 and a lateral magnification of 16. Upon taking apart the outmost shell, I observed that the objective consisted of a larger, about $8.5 \mathrm{~mm}$ diameter, spherical lens paired with a very small, about $4 \mathrm{~mm}$ diameter, aspheric as the front lens. There were also two rings that doubled as support for the larger lens but also as an aperture to block out any laser light that is incident on the face of the objective at a large angle. The top of any microscope objective looks as if it comes to a small point, which also an aperture to better block unwanted light. Using the measurements I took by hand for object distances, I created the lens data editor for these lenses, Fig. 3-12c, and used it to model the disassembled objective as shown in Fig. 3-12a. 

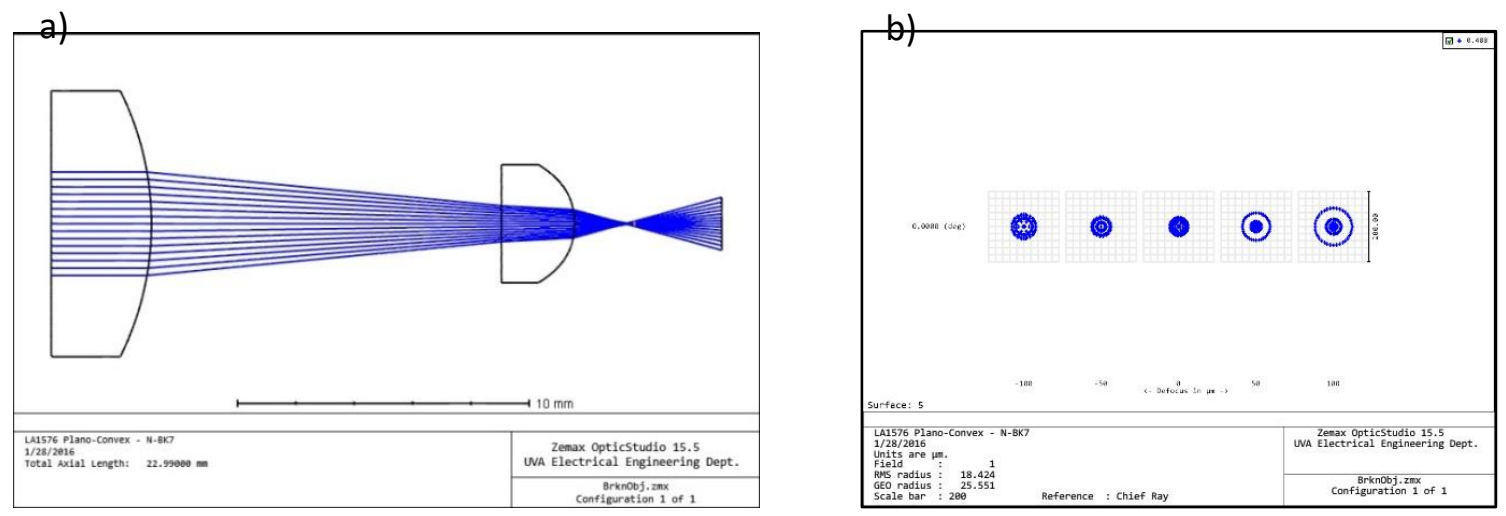

\begin{tabular}{|c|c|c|c|c|c|c|c|c|c|}
\hline \multicolumn{2}{|c|}{ Surf:Type } & \multirow[t]{2}{*}{ Comment } & \multirow{2}{*}{$\begin{array}{l}\text { Radius } \\
\text { Infinity }\end{array}$} & \multirow{2}{*}{$\begin{array}{c}\text { Thickness } \\
\text { Infinity }\end{array}$} & \multirow[t]{2}{*}{ Material } & \multirow[t]{2}{*}{ Coating } & \multirow{2}{*}{$\begin{array}{c}\text { Semi-Diamet } \\
0.000\end{array}$} & \multirow{2}{*}{$\begin{array}{l}\text { Conic } \\
0.000\end{array}$} & \multirow{2}{*}{$\begin{array}{c}\text { TCE } \times 1 \text { 1E-6 } \\
0.000\end{array}$} \\
\hline O OBJECT & Standard $\mathbf{v}$ & & & & & & & & \\
\hline 1 STOP (aper) & Standard $\mathbf{v}$ & pcx, spherical & Infinity & 3.440 & $\mathrm{~N}-\mathrm{BK} 7$ & THORA & $4.500 \mathrm{U}$ & 0.000 & - \\
\hline 2 (aper) & Standard $\mathbf{v}$ & LA1576-A & -10.000 & 12.000 & & THORA & $4.500 \mathrm{U}$ & 0.000 & 0.000 \\
\hline 3 (aper) & Standard $\mathbf{v}$ & & Infinity & 2.500 & D-ZLAF5 ... & & $2.000 \mathrm{U}$ & 0.000 & - \\
\hline 4 (aper) & Even Asphere - & 355151-A & -2.700 & 1.800 & & THORA & $2.000 \mathrm{U}$ & -2.063 & 0.000 \\
\hline 5 IMAGE & Standard $\mathbf{v}$ & & Infinity & - & & & 0.018 & 0.000 & 0.000 \\
\hline
\end{tabular}

Figure 3-12. Disassembled microscope objective data a) cross section ray diagram b) spot diagram c) lens data editor.

The first thing about these lenses to catch the eye is that this combination does not yield a focal point with any kind of blur. As far as the ray diagram can prove, this objective has a definite clear cut focal point, pointing to a need to mix up lens types between spherical and aspherical versus using just one lens type as we had done before. Generating the spot diagram for these lenses, Fig. 3-12b, supports this notion by illustrating an intense, bright focal point with a diameter of 36 microns.

\subsection{Microscope objective design}

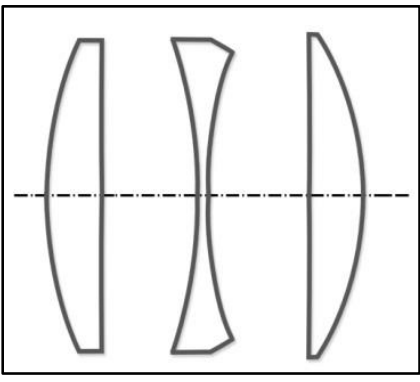

Figure 3-13. Cooke triplet lens diagram. ${ }^{5}$

In taking apart the simple achromatic objective, I was able to better understand the optical spacing and types of lens. Jumping off of the idea to use a spherical and aspherical combination, I began exploring the notion of building a Cooke triplet, shown above in Fig. 3-13. 
The Cooke triplet is the simplest design form with enough degrees of freedom to correct all first and third order aberrations. Generally the two outer elements are positive crowns, have low dispersion, and the middle element is a negative flint, has high dispersion, with an aperture stop located on either side of the middle element to help create a natural waist in the beam and block light that is hitting bottom or sides of the objective at very wide angles ${ }^{7}$. This design helps to control distortion and coma, misalignment of optical elements, as well as lateral color shifts.

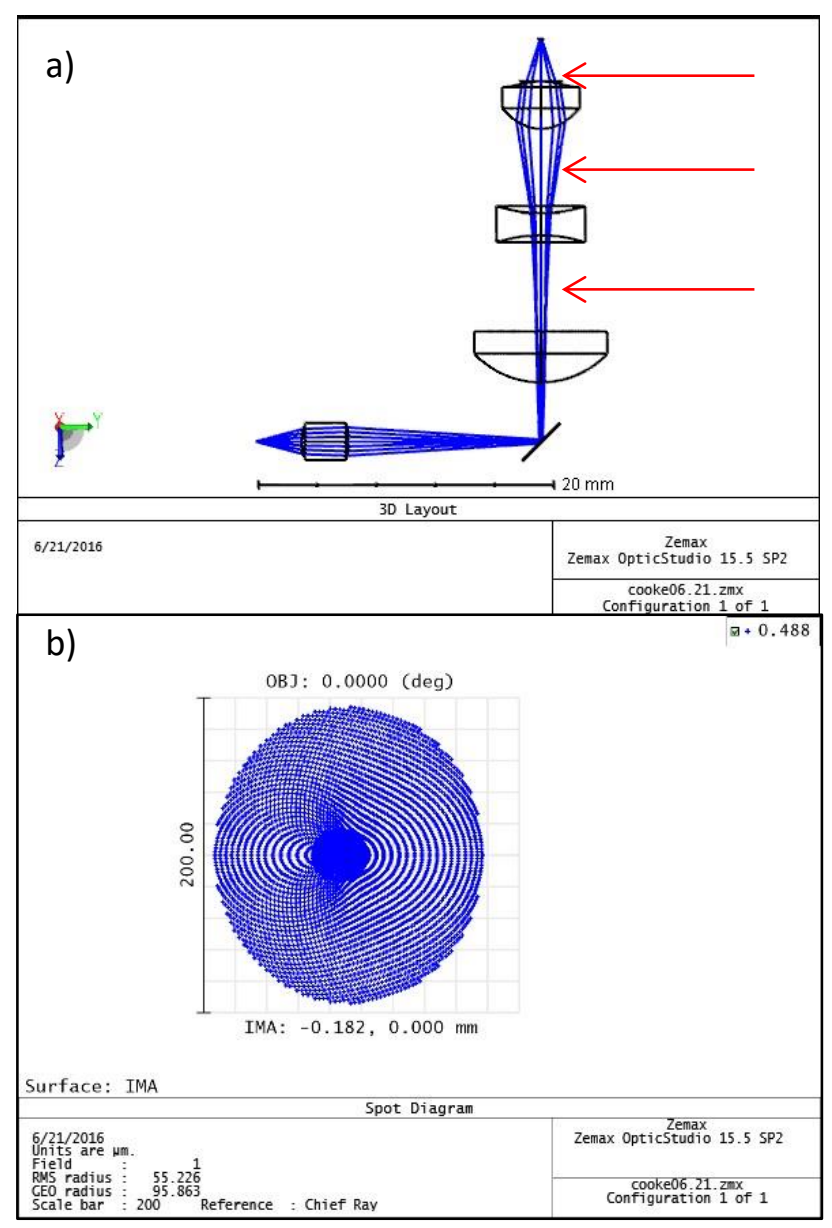

Figure 3-14. Designed Cooke triplet laser to chip a) ray diagram b) spot size. 
The first element, from the left, is usually a spherical lens, the middle is normally a biconcave lens and the third an aspheric lens ${ }^{5}$. The ray diagram of my triplet, following the laser to channel path is shown below in Fig. 3-14a. The origin, or source, is the 488nm laser diode in the bottom left corner. The rectangular slab is representative of the $3 \mathrm{~mm}$ right angle prism that will also sit inside the objective tube. There are three apertures, indicated with the red arrows in the ray diagram. Inserting a top aperture will help alleviate the "fuzz" surrounding the high intensity spot, about 40 microns in diameter, at chip, shown in Fig. 3-14b.

The ray diagram and $3 \mathrm{~d}$ model of my triplet, following the channel to detector path is shown below in Fig. 3-15a and Fig. 3-15b. The origin is now the top, right corner, using the $510 \mathrm{~nm}, 590 \mathrm{~nm}$, and $630 \mathrm{~nm}$ wavelengths as placeholders for the various florescent dyes (Note: the orange is just difficult to see around the red). The beam appears to travel perfectly fine, even after being turned by the right angle prism and then turned again by the dichroic mirror towards the detector.
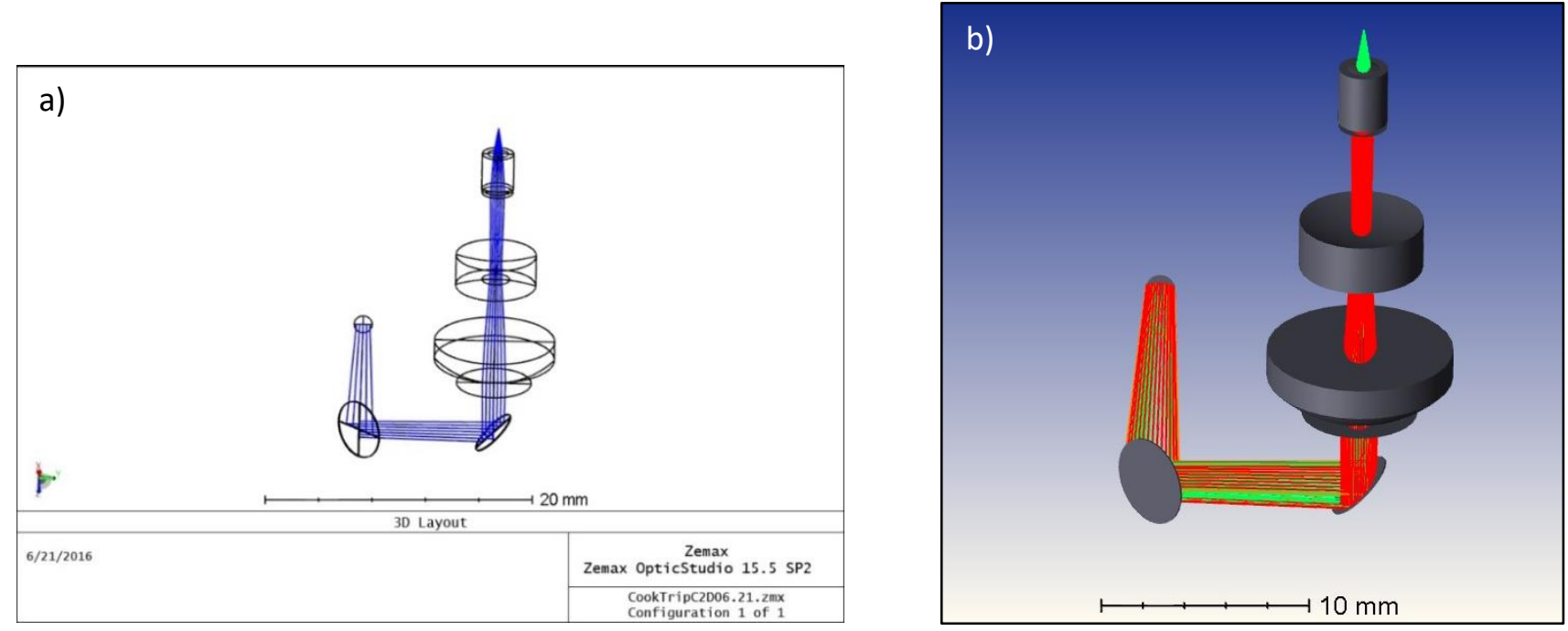

Figure 3-15. Designed Cooke triplet chip to detector a) ray diagram b) 3D with fluor colors. 
With the pre-simulations successfully completed, I was now ready to build an actual Cooke Triplet. I purchased the spherical lens (diameter $9 \mathrm{~mm}$ and $\mathrm{f}=12 \mathrm{~mm}$ ), biconcave lens (diameter $6 \mathrm{~mm}$ and $\mathrm{f}=-6 \mathrm{~mm}$ ), aspheric lens (diameter $6.2 \mathrm{~mm}$ and $\mathrm{f}=3.1 \mathrm{~mm}$ ), along with an objective tube preset with retaining rings, shown fully put together in Fig. 3-16, and a right angle prism dielectric mirror (length $10 \mathrm{~mm}$ ) from Thorlabs. I initially set up a simplified breadboard system using the extra equipment, shown in Fig. 3-17, before using the multi-color system 2, nicknamed Condor, for all further testing. As mentioned when building the cylindrical lens test setup, all of the optical components were capable of being much closer together, the mounts just needed to be changed in order to address that. In my test configuration shown in Fig. 3-17, I've outlined the drastic size change with a yellow arrow. The width of the test system was $115 \mathrm{~mm}$ long and the height of the objective stack was $65 \mathrm{~mm}$, about 2.5 inches, and drastically reduced from the previous six inch tall objective stack.
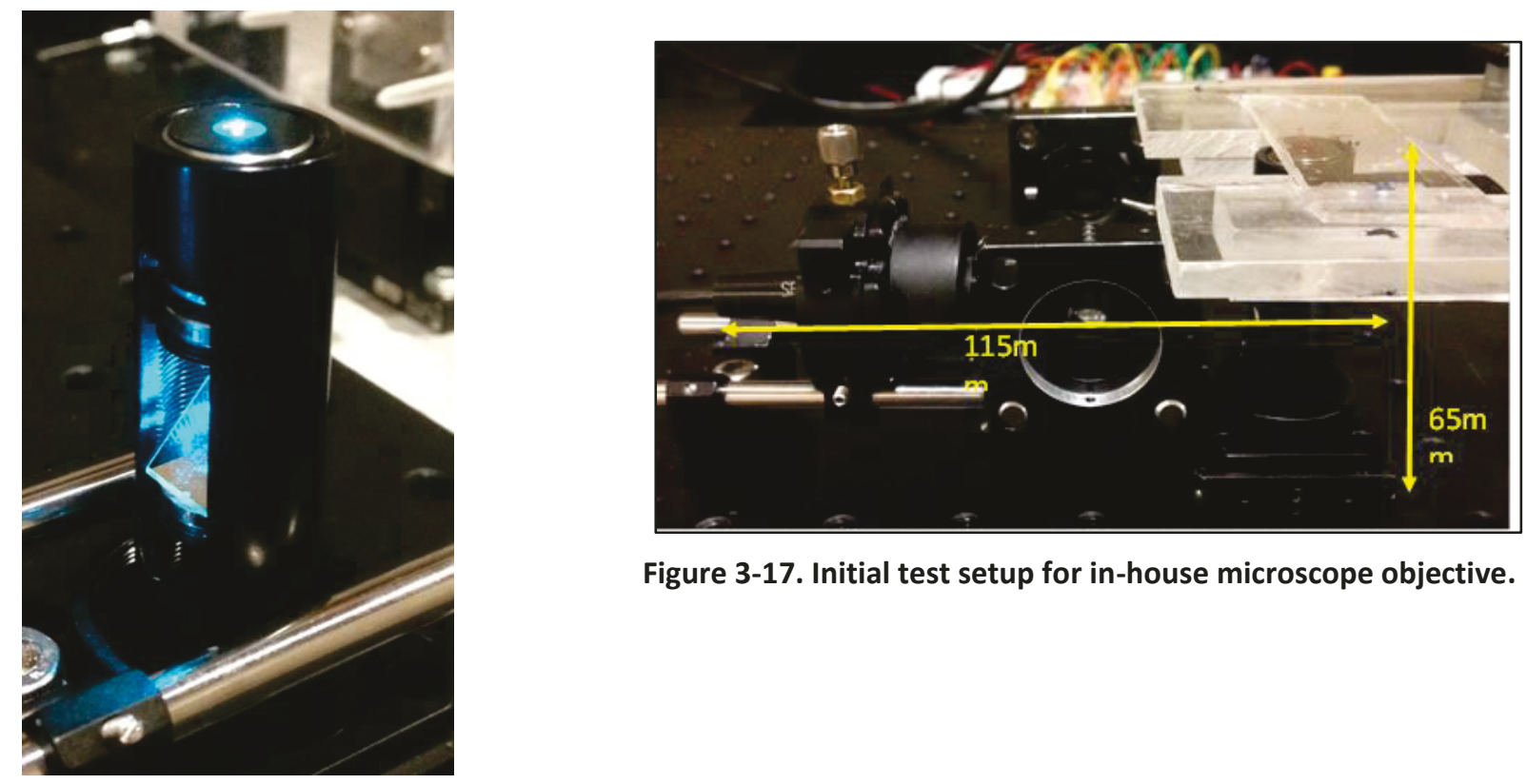

Figure 3-17. Initial test setup for in-house microscope objective.

Figure 3-16. Fully assembled in-house microscope objective. 
The final experiment before moving my triplet over to the Condor system was a test in how much the beam diameter does, or does not, change from the chip to the detector given that the emitted light must turn twice as mentioned above when I modeled the channel to detector beam pathway, and it may need to travel to the very back of the MPMT depending on color. In order to perform the experiment, the objective was used to focus the emission beam right after turning from the dichroic mirror and passing through the pinhole, about $20 \mathrm{~mm}$. From there the focus was held constant while the detector was moved out along its z-axis at $25 \mathrm{~mm}$ increments; at each value calipers were used to accurately measure the diameter of the beam, in $\mathrm{mm}$, on the detector. A diagram of the procedure and distances is shown in Fig. 3-18, while the distances versus beam diameter in $\mathrm{mm}$ is graphed in Fig. 3-19. The graph shows a purchased, manufactured objective, the designed objective and the simulated objective side by side. In general, they all follow the same trend and suggest that our beam is diverging a little too much as it reaches the back of the detection optics inside the detector.

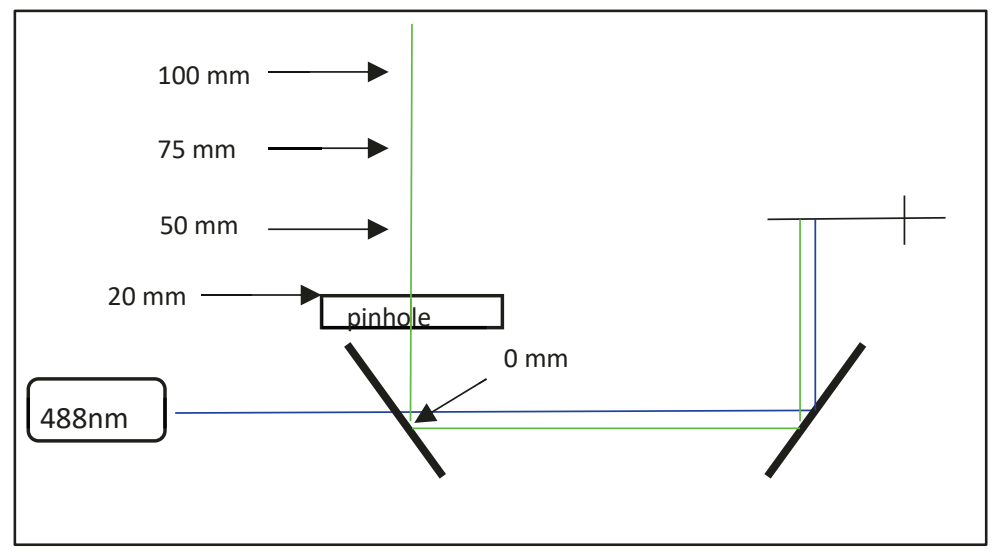

Figure 3-18. Depiction of distances where beam diameter measurements were taken from chip to detector.

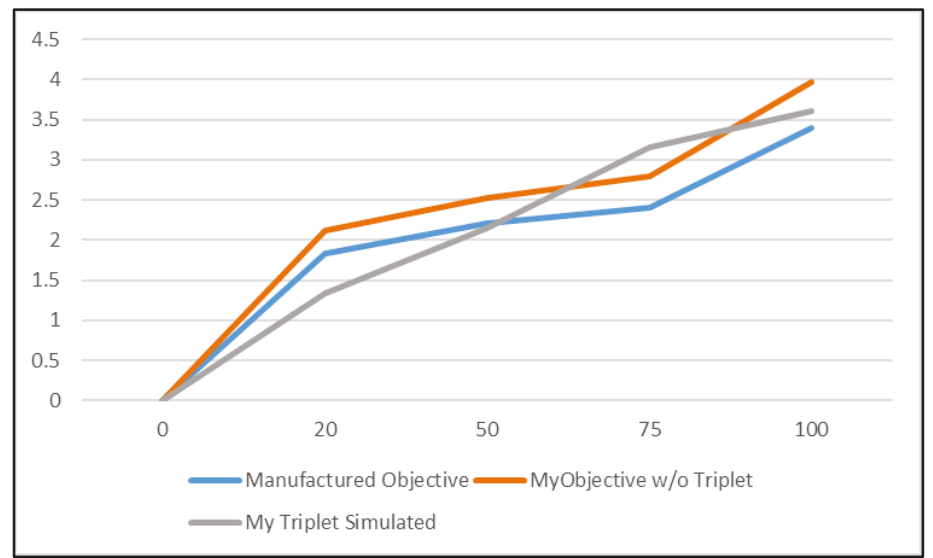

Figure 3-19. Distance from dichroic mirror versus emission beam diameter. 


\subsubsection{Microscope objective modeling iterations}

The designed triplet was integrated into the third multi-color system, using the linear array multi-channel mPMT and the mechanical and optics design depicted at the end of Chapter 2. The first iteration of Condor system optics was modeled as in Fig. 3-20a, with the corresponding spot diagram shown in Fig. 3-10b. The beam fit into the channel but experienced a terrible "fish-eye" effect, indicative of the focal point starting behind the chip, such that the objective is always "too" focused. The "fish-eye" effect is noted in the spot diagram as well given that the distance between rings gets larger as we move out of the diagram.
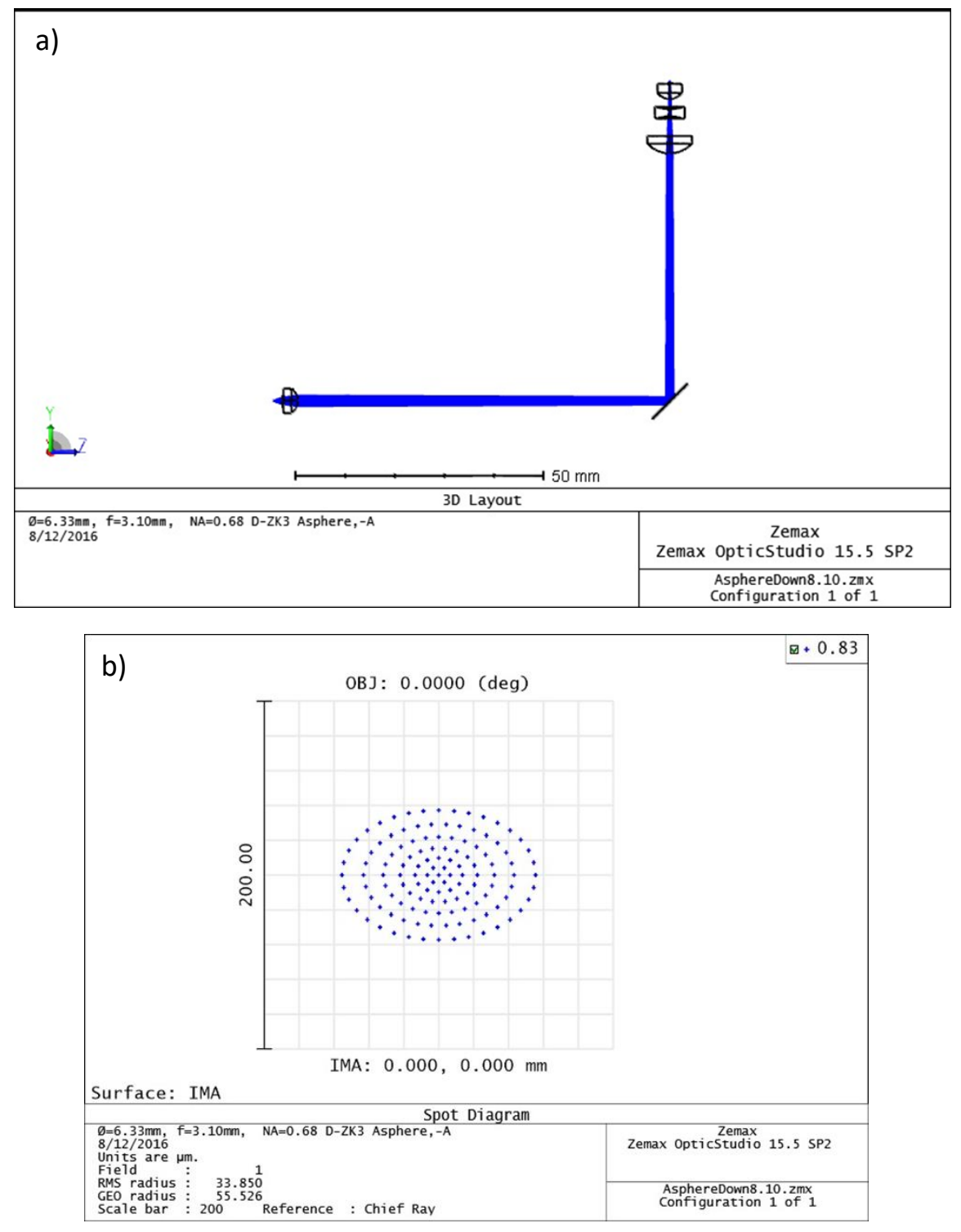

Figure 3-20. First iteration of Condor optics a) ray diagram model b) spot size. 
We noticed that the focus seemed better the closer we placed the bottom lens to the rest of the lens stack; therefore, the triplet was rearranged to be very close as shown in Fig. 3-21a with its corresponding spot diagram shown in Fig. 3-21b. This second iteration again fit the channel quite nicely, without any aberrations appearing; however, its focal distance was 2.4 $\mathrm{mm}$ from the chip, which is much too far for collection purposes.
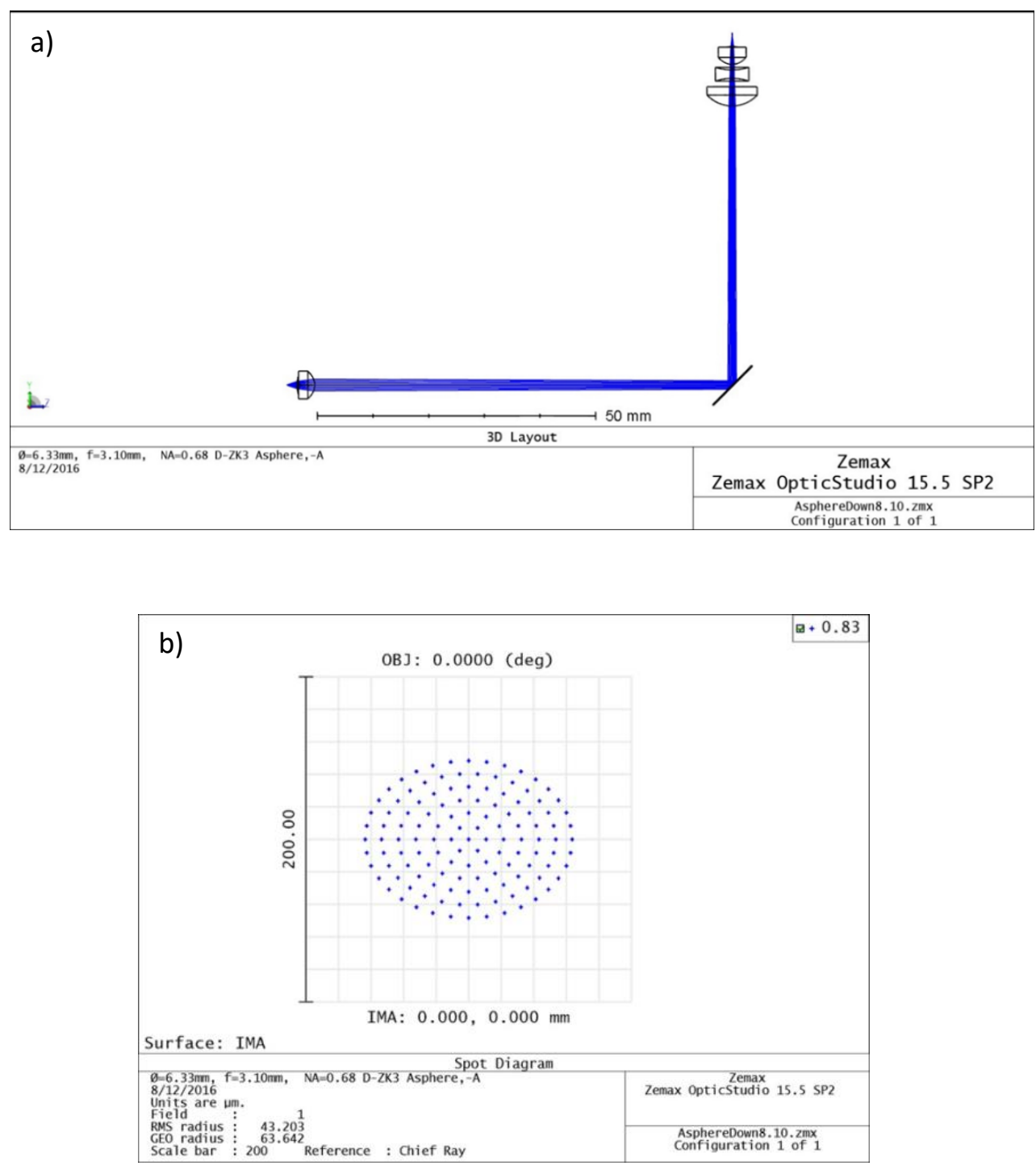

Figure 3-21. Second iteration of Condor optics a) ray diagram model b) spot size. 
Knowing the aspheric lens gives us the ability to adjust and focus the laser diode in any way the system needs, we decided to try the same design with the detection path. This this iteration consisted of just the aspheric lens as modeled in Fig. 3-22a. The lens was shifted down inside the objective tube by $2 \mathrm{~mm}$ so that the chip could rest directly on top when in focus. The corresponding spot diagram, with a diameter of 80 microns, shown in Fig. 3-22b, was the most encouraging to date. Not only did the excitation beam fill the channel and objective the way we would expect of commercial objectives, but the spot diagram showed the flattest field to date, without any evidence of field curvature present.
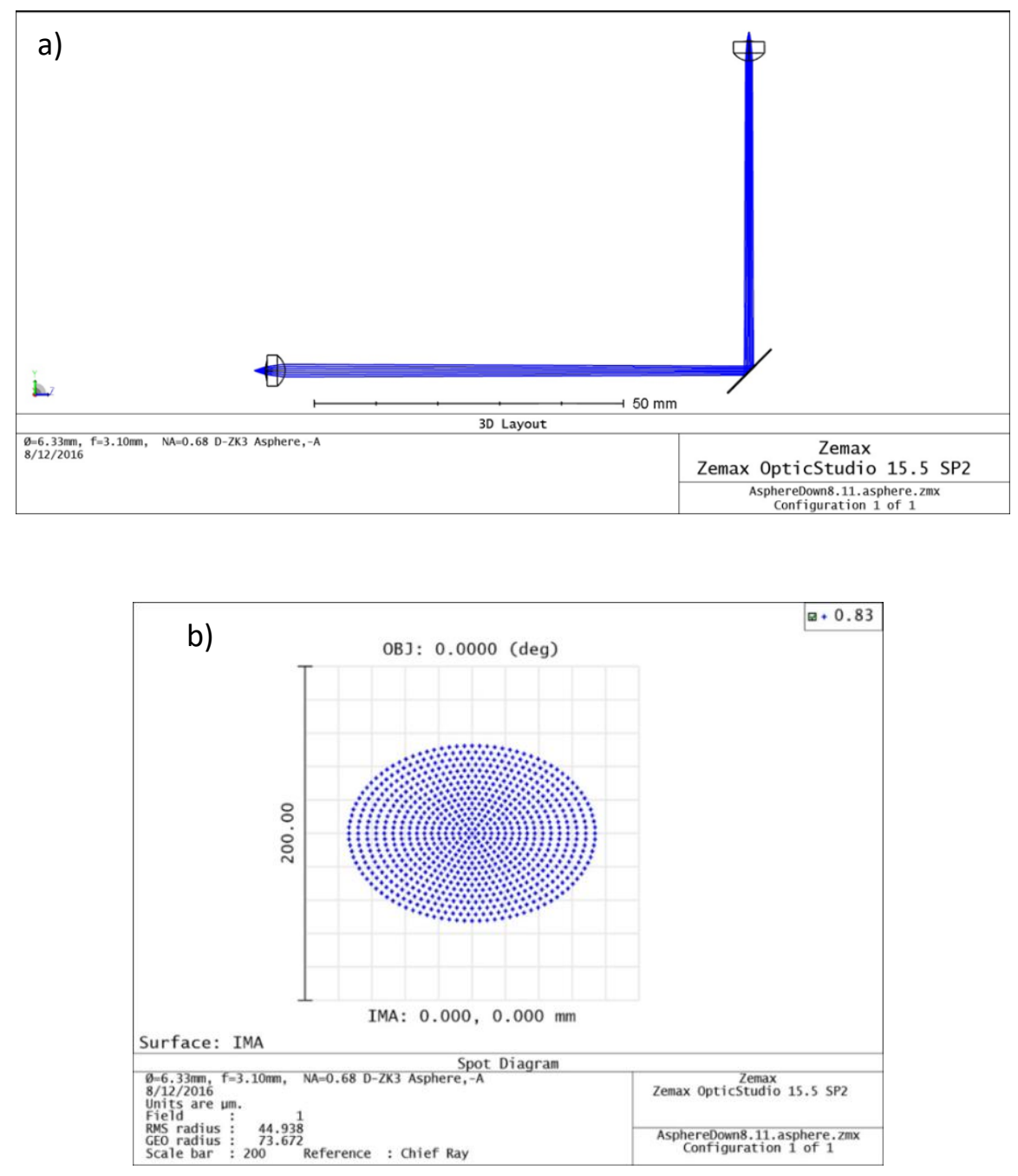

Figure 3-22. Third iteration of Condor optics a) ray diagram model b) spot size. 
After the success of this experiment, we decided to continue pursuing the option of using just a single aspheric lens above the right angle dichroic mirror as our complete objective stack. The original triplet would have been successful with small adjustments to each lenses spacing, but since the system doesn't demonstrate strong chromatic aberrations, the appeal in using just one lens vastly outweighs the need to correct the minimal aberrations. In addition to lens aberrations, the realistic numerical aperture and working distance that could be achieved using just an aspheric lens over the triplet needed to be weighed. In the end, it was decided that the sensitivity of the system wouldn't be strongly affected by a smaller numerical aperture and that the decreased working distance might in fact increase amount of light collected. With this in mind, three different aspheric lenses were ordered, from Thorlabs, to compare in performance to the one used in our original experiments. The critical parameters for each of those lenses is listed in Table 3-1, with the limit of detection, calculated and provided by Dan Nelson from the data collection, listed in the very last column.

Table 3-1. Comparison of potential aspheric lens for Condor optics.

\begin{tabular}{|c|c|c|c|c|c|}
\hline Lens & Diameter & Focal Distance & $\begin{array}{c}\text { Working } \\
\text { Distance }\end{array}$ & $\begin{array}{c}\text { Numerical } \\
\text { Aperture }\end{array}$ & Calculated LOD \\
\hline 354330-A & $6.2 \mathrm{~mm}$ & $3.10 \mathrm{~mm}$ & $1.76 \mathrm{~mm}$ & 0.68 & $2.00 \mathrm{E}-10$ \\
\hline C140TMD-A & $2.4 \mathrm{~mm}$ & $1.45 \mathrm{~mm}$ & $0.81 \mathrm{~mm}$ & 0.58 & $3.49 \mathrm{E}-10$ \\
\hline C710TMD-A & $2.7 \mathrm{~mm}$ & $1.49 \mathrm{~mm}$ & $0.42 \mathrm{~mm}$ & 0.53 & $8.20 \mathrm{E}-10$ \\
\hline C392TME-A & $4.0 \mathrm{~mm}$ & $2.75 \mathrm{~mm}$ & $0.98 \mathrm{~mm}$ & 0.64 & $1.70 \mathrm{E}-10$ \\
\hline
\end{tabular}

The first and last lenses provided the best limits of detection and focal point diameter and shape within the channel. Ultimately, the last lens listed was chosen for the final objective design as we decided that a slightly smaller numerical aperture was worth the significantly smaller working distance, allowing the chip to sit directly on top of the objective. The lens also 
provided the perfect spot size, estimated at about 60 microns, for the automatic alignment team. The final channel width was 80 microns, so having a spot size that would fit completely inside the channel would allow us to find to know with confidence when we were out of focus or hitting and reflecting off the channel walls.

The lens also provided a great deal more clarity when focusing into the channel, by adjusting the height or z-direction, when compared to the spherical lens from our basic table top setup. The process for finding the center of the channel in focus is depicted in the panel of photos in Fig. 3-23. Panel A shows the chip and channel loaded without any adjustments. Panel B shows the channel starting to expand as the objective is moved in the positive $z$-direction by 5 micron steps. Panel $\mathrm{C}$ shows an even higher z-position for the objective where we have centered the beam within the walls of the channel. Panel D shows a bent band of intensity along the far right of the photo meaning that as we scan adjust in the x-direction we have hit the left wall. Panel E shows a bent band of intensity along the far left of the photo meaning that the objective spot is reflecting off the right wall. Finally, panel F depicts what we expect to see when the objective is filled correctly and the spot size is perfectly centered inside the chip channel, not touching the walls or back of the chip.
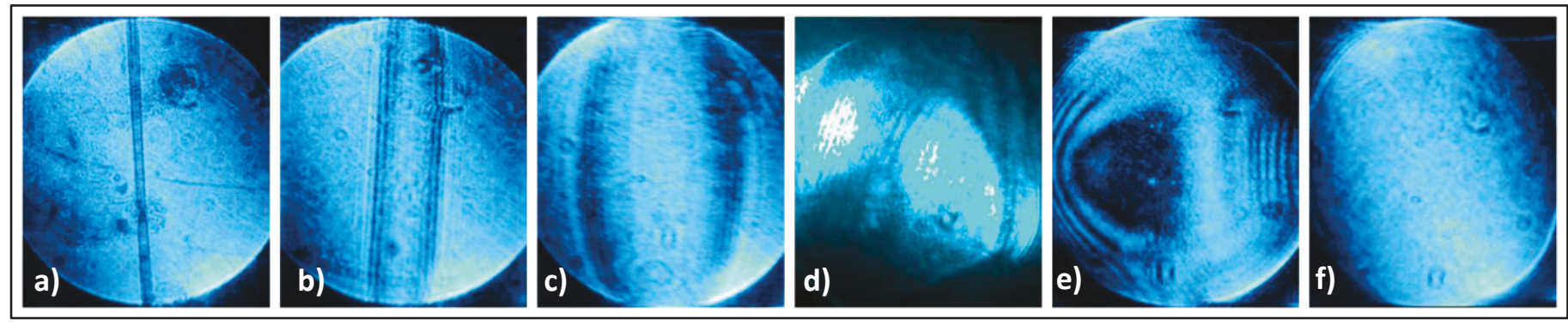

Figure 3-23. In-house objective focusing into chip. 


\section{Chapter 4. Conclusions and future work}

\subsection{Summary}

The goal of this work was to create an optical detection system that could perform ultrarapid DNA screening in a turnkey manner for any user, which fit into a one foot by one foot area (weighed 30 pounds or less) and held the capability to be further miniaturized into the shape and size of a portable CD player. Initially, I studied the two most likely detectors for our new system, an avalanche photodiode or micro photomultiplier tube. The avalanche photodiode had low dark currents in the $10^{-11}$ ampere range for reverse-bias voltage up to $120 \mathrm{~V}$. It also had good responsivity at $550 \mathrm{~nm}$ with peak quantum efficiency of $80 \%$ at $600 \mathrm{~nm}$ and a corresponding gain of about 100 . In comparison, the micro photomultiplier tube exhibited lower efficiency, about $20 \%$ at $550 \mathrm{~nm}$ and higher dark current in the nA range. On the other hand, it also achieved gains greater than $3.0 \times 10^{\wedge} 6$ ultimately allowing for detection of very small signals, $10^{\wedge}-10$, which led to its use in all of the integrated systems.

Concurrently, I modeled and characterized various optical lens designs for both the laser to channel pathway and the channel to detector path. At first, the only goal of the lens design was to ensure that a viable signal could be retrieved using the bare minimum number of optics. Once this was achieved, I investigated combinations of aspheric lens and dichroic mirror and filter setups in order to maximize signal, while maintaining as few lens as possible. In addition, I designed our own microscope objective, altering the design until we were able to achieve a spot size of 60 microns, in order to fit within an 80 micron microfluidic channel, with minimal loss in power, measuring about $25 \mathrm{~mW}$ at the channel. 
With this final set of results, we implemented all the updated optical components,

including lenses, mirrors and filters into the third integrated system using the linear array multichannel microPMT as well as both lasers, the $488 \mathrm{~nm}$ excitation diode and the $638 \mathrm{~nm}$ laser valve diode. This excitation and detection sub-system was integrated with the rest of the mechanical and electrical design to form the fully integrated year end prototype. This assembly stacked the electrical boards and power supply underneath the bulk of the equipment, shown in Fig. 4-1, giving it more height but allowing it to take up much less area. The top down view, shown in Fig. 4-2, details how the mechanical components were all designed around the chip, which rests in the center, with the optics, excitation and detection equipment resting on the lower left hand side. The details of the power budget are summarized and diagramed in Fig. 43, showing the various power measurements, gain, quantum efficiency and ultimate limit of detection achieved on the prototype assembly.

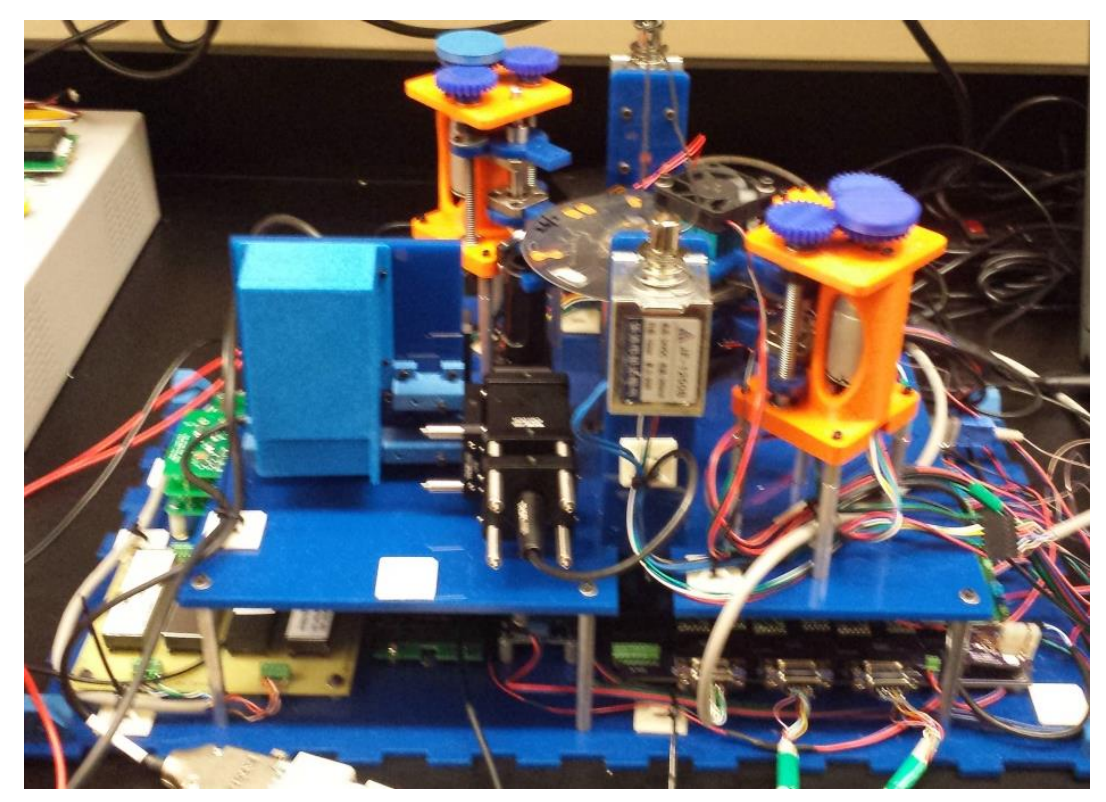

Figure 4-1. Year-end prototype assembly - Raptor. 


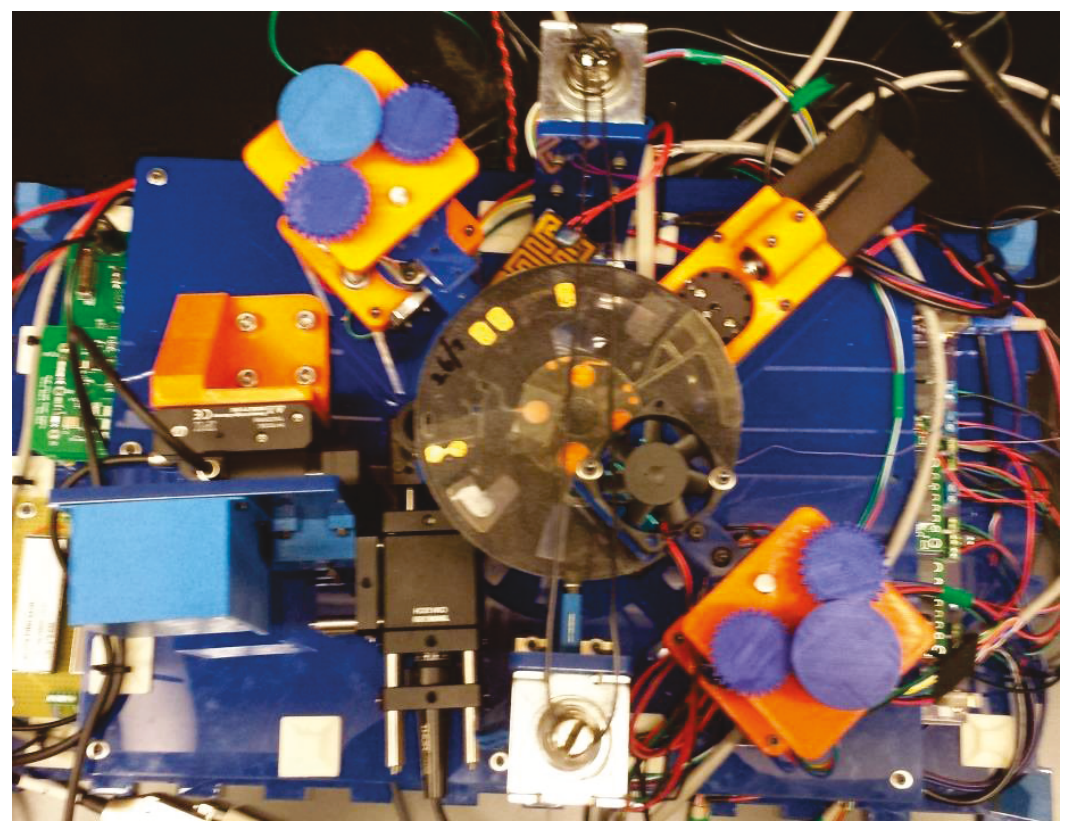

Figure 4-2. Top-down view of year-end prototype.

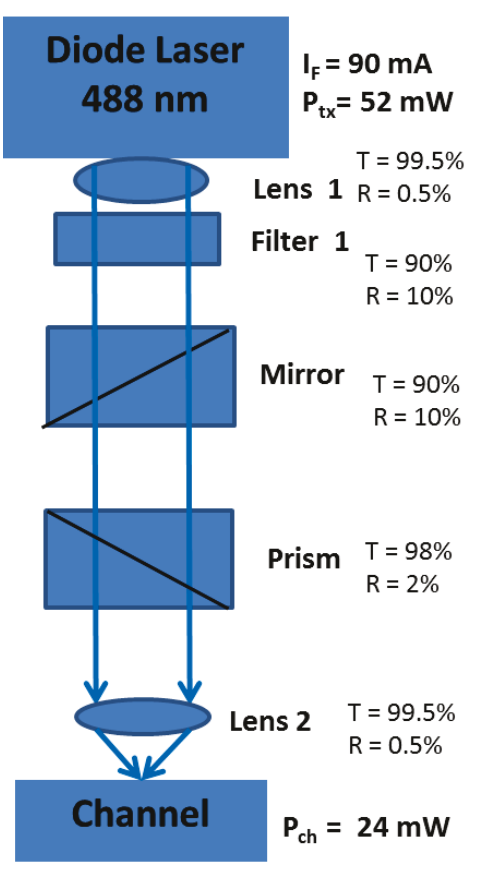

Concentration: $10^{\wedge}-6-10^{\wedge}-12$ Absorption: $73000 \mathrm{~cm}^{-1} \mathrm{M}^{-1}$ Power absorbed: 23\% Emitted power $\mathrm{P}_{\mathrm{e}}$ : $24 \mathrm{~mW}$

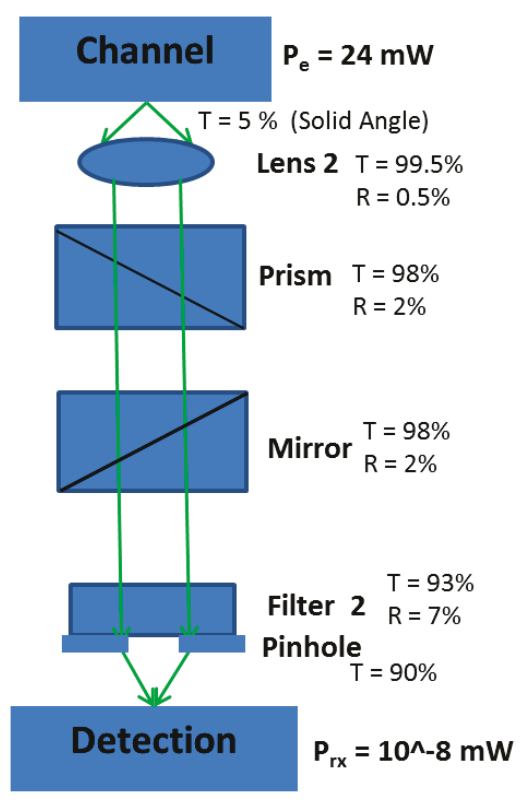

Power absorbed: 11-12\%

Emitted power $P_{\mathrm{rx}}: 10^{\wedge}-8 \mathrm{~mW}$ Gain \& QE: 3.0x10^6 and 20\%@500nm Dark Current: 1 nA LOD: $1.70 \times 10^{\wedge}-10$

Figure 4-3. Power budget data for year-end prototype. 


\subsection{Future Work}

This section addresses potential pathways to further reducing the size of all the optical components within the system. While this prototype DNA assay machine can distinctly measure fluorescein emission, for multiple different colors, with a commercial level of sensitivity and limit of detection, the overall footprint can still be smaller.

One way of achieving this would be through the use of microspheres: lenses with a diameter of about one micron and focal length of about $630 \mathrm{~nm}^{11}$. They are especially convenient for coupling light into or out of fibers, but could certainly be used for relaying information if our system were to be more compact. The focal point is so small that they can virtually be placed directly in contact with their intended surfaces reducing the amount of open air space within the beam paths. These microlenses are also crafted into aspheric lenses, especially designed to collect and collimate laser diode radiation, with a diameter of 200 microns and focal length of about $780 \mathrm{~nm}^{11}$, making them ideal replacements for the aspheric lens and collimating tube within the current prototype. Each type of lens can be made in a variety of materials, specific sizes and numerical apertures. They can also be placed in specialized holders or attached directly onto the component of interest.

This type of lensing was not implemented in the current prototype because a great deal of the system's mechanical and electrical components would also need to decrease in size before decreasing the optics again, from $25 \mathrm{~mm}$, would be logical. Once the electrical power boards and solenoid motors decrease in size, this would be the next step in minimizing optics. 


\section{References}

[1] Anderson, Neil, Prashant Prabhat, and Turan Erdogan. "Spectral Modeling In Fluorescence Microscopy." Semrock White Paper Series (2014).

[2] Davidson, Michael W. "Introduction to Microscope Objectives." MicroscopyU. Nikon, 2016.

[3] "Fluorescence Fundamentals." Thermo Fisher Scientific. Molecular Probes Handbook, 2016.

[4] Geary, Joseph M. Introduction to Lens Design with Practical ZEMAX Examples. Richmond, VA: Willmann-Bell, 2002.

[5] Jenny, Reinhard. "Fundamentals of Optics - An Introduction for Beginners." Volpi Manufacturing USA (2001).

[6] Lakowicz, Joseph R. Principles of Fluorescence Spectroscopy. New York: Springer, 2010.

[7] Olson, Craig, and Julie Bentley. Field Guide to Lens Design. N.p.: SPIE / International Society for Optical Engineering, 2012.

[8] "Photomultiplier Tubes: Basics and Applications." Hamamatsu Handbook, 2006.

[9] Pierret, Robert F. Semiconductor Device Fundamentals. Reading (Ma.): Addison-Wesley, 1996.

[10] Pollock, Clifford R. Fundamentals of Optoelectronics. Chicago: Irwin, 1995.

[11] OptoSigma. Optical Components: Microspheres, 2015.

[12] Shrinivasan, Sushil, Pamela M. Norris, James P. Landers, and Jerome P. Ferrance. "A Low-Cost, Low-Power, Consumption Miniature Laser-Induced Fluorescence System for 
DNA Detection on a Microfluidic Device." Journal of the Association for Laboratory Automation 11.4 (2006): 254-59.

[13] "Tag Archives: Slope Efficiency." Laser Diode Selection. SPIE, Kokyo Inc., 2014.

[14] Tan, Eugene, Rosemary S. Turingan, Catherine Hogan, Sameer Vasantgadkar, Luke Palombo, James W. Schumm, and Richard F. Selden. "Fully Integrated, Fully Automated Generation of Short Tandem Repeat Profiles." Investigative Genetics 4.1 (2013): 16.

[15] Young, lan T., Yuval Garini, Heidi R. C. Dietrich, Wim Van Oel, and Guus Liqui Lung. "LEDs for Fluorescence Microscopy." Three-Dimensional and Multidimensional Microscopy: Image Acquisition and Processing XI 5324 (2004).

[16] Osram Opto Semiconductors. Cyan Diode, TO56 (PLT5 488) Datasheet, 2014. 